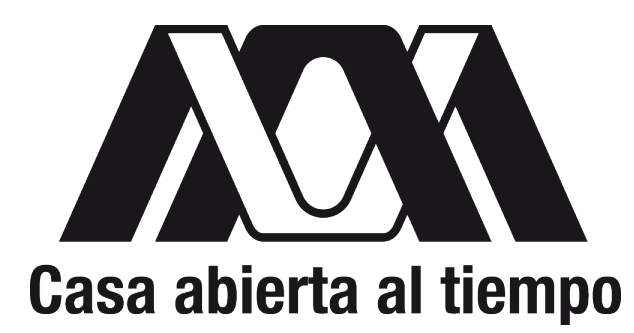

UNIVERSIDAD AUTÓNOMA METROPOLITANA

UNIDAD IZTAPALAPA

DIVISIÓN DE CIENCIAS BÁSICAS E INGENIERÍA

TESIS:

\title{
PROPIEDADES TOPOLÓGICAS DEL TIPO ESTRELLA P
}

Presentada por:

\section{Luis Enrique Gutiérrez Domínguez}

Para obtener el grado de:

\section{Maestro en Ciencias (Matemáticas)}

Director de tesis:

Dr. Richard Gordon Wilson Roberts

Sinodales:

Dr. Richard Gordon Wilson Roberts

Dr. Rodrigo Jesús Hernández Gutiérrez

Dr. Ángel Tamariz Mascarúa

Iztapalapa, Ciudad de México, 27 de octubre del 2020. 

Dedicado a mi abuelita 


\section{Resumen}

El presente trabajo se centra en el estudio de algunas propiedades topológicas tipo compacidad llamadas propiedades estrella $P$. Dado un espacio topológico $X$ y una propiedad topológica $P$, decimos que el espacio $X$ es estrella $P$ si para cualquier cubierta abierta $\mathcal{U}$ de $X$ podemos encontrar un subconjunto $A$ con la propiedad $P$ tal que $\operatorname{St}(A, \mathcal{U})=X$. El subconjunto $A$ se llama núcleo para la cubierta $\mathcal{U}$. El objetivo de esta tesis es presentar condiciones suficientes y necesarias para que dos clases de espacios topológicos sean equivalentes; es decir, dadas dos propiedades topológicas $P$ y $Q$, estudiaremos en qué clases de espacios ser estrella $P$ es equivalente a ser estrella $Q$. También se presentan las relaciones que existen entre la propiedad estrella $P$ y propiedades como compacidad numerable, pseudocompacidad, tenuemente Lindelöf, entre otras; por ejemplo se dan condiciones suficientes y necesarias para que la clase de los espacios estrella numerable y la clase de los espacios tenuemente Lindelöf sean equivalentes. En los casos donde solamente tengamos la suficiencia proporcionaremos ejemplos que diversos autores han construido para evidenciar que las clases de los espacios no son equivalentes. Estos ejemplos son de gran importancia ya que nos ayudan a cuestionar bajo qué condiciones las clases de estos espacios podrían ser equivalentes.

En la parte final del trabajo se presentan dos propiedades más débiles que la propiedad estrella $P$, a las que denominamos estrellas débiles. En esta sección del trabajo se examina en qué clases de espacios topológicos estas dos propiedades son equivalentes y la relación que poseen con la propiedad estrella $P$ y con otras propiedades tipo compacidad. 


\section{Introducción}

El primer capítulo de este trabajo contiene resultados preliminares importantes para el desarrollo del estudio de las propiedades estrella $P$, consta de teoremas importantes sobre espacios pseudocompactos y numerablemente compactos. Además, se construye un espacio que se ocupará constantemente para demostrar la no equivalencia entre clases de espacios topológicos; este espacio es el espacio de Mröwka determinado por familias casi ajenas en algún cardinal infinito $\kappa$.

En el capítulo 2 se introduce la propiedad estrella $P$ y características elementales sobre ésta. El primer resultado relevante de este capítulo es que cualquier espacio topológico numerablemente compacto es estrella finito, pero la implicación recíproca no se cumple, ya que existe un espacio $T_{1}$ estrella finito que no es numerablemente compacto, demostraremos que en la clase de los espacios de Hausdorff estas dos propiedades son equivalentes. Además definiremos lo que es un espacio $n$-estrella finito y probaremos que un espacio topológico estrella compacto es 1-estrella finito, pero que la implicación recíproca no se cumple. En la segunda sección del capítulo 2 se muestra que en la clase de los espacios de Tychonoff cada espacio estrella finito de orden $\omega$ es pseudocompacto y que cada espacio tenuemente compacto es 2-estrella finito. Otros resultados importantes que se exponen en este capítulo son que el producto de un espacio estrella Lindelöf y un espacio compacto es un espacio estrella Lindelöf y que el producto de un espacio tenuemente Lindelöf y un espacio separable es un espacio tenuemente Lindelöf.

En el capítulo 3 se estudia la propiedad estrella $P$ cuando " $P=$ numerable" y cuando " $P=$ finito", en la primera parte del capítulo se estudian relaciones entre estas y otras propiedades del tipo compacidad. Se muestra que en la clase de los espacios $T_{1}$, cada espacio estrella Lindelöf es tenuemente Lindelöf y también se prueba que el producto de un espacio de Lindelöf y un espacio numerablemente compacto no necesariamente es un espacio estrella Lindelöf. En general, en esta primera sección se demuestran una serie de implicaciones de propiedades del tipo estrella $P$ las cuales no pueden ser reversibles en la clase de los espacios $T_{1}$. Por ejemplo en el capítulo 2 se muestra que cada espacio $T_{1}$ con extensión numerable es estrella numerable y en este capítulo se proporciona un ejemplo que demuestra que la implicación recíproca no se cumple. Este ejemplo es el mencionado anteriormente, el espacio de Mröwka $\Psi(\mathcal{A})=\omega \cup \mathcal{A}$ que es separable y tiene extensión no numerable. El teorema más importante de esta sección fue demostrado 
en [3] que nos proporciona condiciones suficientes y necesarias para que la clase de los espacios con extensión numerable y la clase de los espacios tenuemente Lindelöf sean equivalentes. Otro resultado interesante de esta sección es la existencia de un $P$-espacio de Tychonoff tenuemente Lindelöf que no es estrella Lindelöf bajo la suposición de que existe una familia $M C A G \mathcal{A}$ en $\omega_{1}$ tal que $|\mathcal{A}|^{\omega}=|\mathcal{A}|$, esta suposición es independiente de ZFC. La segunda sección de este capítulo está centrada en el estudio de los espacios $S C E$ que son aquellos que para cada cubierta abierta se puede encontrar un núcleo con extensión numerable, estos espacios fueron estudiados ampliamente en [18]. La clase de los espacios $S C E$ yace entre la clase de los espacios estrella Lindelöf y la clase de los espacios tenuemente lindelöf.

El capítulo 4 está dedicado a las propiedades estrella $P$ en la clase de los $P$-espacios y en la clase de los espacios de Moore. En la primera sección se define un espacio débilmente $\kappa$-metaLindelöf y se da una equivalencia entre la clase de los espacios con extensión numerable y la clase de los espacios estrella numerable. Además se proporciona un teorema que nos provee de la equivalencia de las siguientes propiedades en la clase de los $P$-espacios normales: estrella numerable, estrella Lindelöf, tenuemente Lindelöf, la dccc y extensión numerable. En esta sección también se da un ejemplo de un espacio de Hausdorff con la $d c c c$ que no es tenuemente Lindelöf. En la sección 2 de este capítulo se muestra que las propiedades estrella Lindelöf y estrella numerable que fueron estudiadas en el capítulo 3 son equivalentes a la propiedad de separabilidad en la clase de los espacios de Moore.

En el capítulo 5 se estudia la propiedad estrella $P$ debilitada, es decir, las propiedades casi estrella $P$ y débilmente estrella $P$. En la primer sección se muestran las relaciones entre éstas y la propiedad $D C\left(\omega_{1}\right)$. Por ejemplo se prueba que cualquier espacio topológico $T_{1}$ con la $D C\left(\omega_{1}\right)$ es débilmente estrella numerable y que cada espacio con celularidad numerable es débilmente estrella numerable. Además se proporciona un ejemplo de un espacio de Tychonoff que es casi estrella numerable pero no es estrella numerable. Un resultado importante en esta sección nos da condiciones suficientes y necesarias para que las siguientes clases de espacios sean equivalentes: débilmente estrella numerable, casi estrella numerable, $X$ tiene un subespacio denso con extensión numerable y estrella numerable. En la sección 2 de este capítulo se demuestran proposiciones análogas para " $P=$ finito". En la última sección se relacionan las propiedades débiles con propiedades como ser metacompacto y ser metaLindelöf y se demuestra que un espacio de Hausdorff débilmente estrella numerable con diagonal de orden 4 tiene cardinalidad a lo más $2^{\omega}$. 


\section{Índice}

1 Pseudocompacidad y compacidad numerable. 1

1.1 Propiedades básicas y ejemplos. . . . . . . . . . . . . . . . . 1

1.2 Caracterizaciones de pseudocompacidad. . . . . . . . . . . . 4

2 Propiedades estrella P y pseudocompacidad. 13

2.1 Espacios estrella P. . . . . . . . . . . . . . . . . . . . . . . . 13

2.2 Relaciones entre espacios pseudocompactos y espacios estrella P. . . . . 21

3 Espacios estrella P y la propiedad de Lindelöf. 25

3.1 Espacios estrella numerable y estrella Lindelöf . . . . . . . . . . . . . . . 25

3.2 Espacios estrella extensión numerable. . . . . . . . . . . . 36

4 P-espacios y espacios de Moore. 49

$4.1 \quad$ P-espacios . . . . . . . . . . . . . . . . . . . . . . . . . . . 49

4.2 Estrella $\mathrm{P}$ en espacios de Moore. . . . . . . . . . . . . . . 54

5 Estrellas débiles. $\quad 57$

5.1 Estrella débil y casi estrella. . . . . . . . . . . . . . . . . 57

5.2 Espacios débilmente estrella finita. . . . . . . . . . . . . . . 65

5.3 Espacios metacompactos y metaLindelöf. . . . . . . . . . . . . 66

6 Conclusiones $\quad 71$

$\begin{array}{ll}\text { Bibliografía } & 75\end{array}$ 


\section{Capítulo 1}

\section{Pseudocompacidad y compacidad numerable.}

\subsection{Propiedades básicas y ejemplos.}

En este trabajo $\mathbb{R}$ denotará al conjunto de los números reales con la topología Euclideana a menos que se especifique otra cosa. Para cada espacio topológico $X$ denotaremos por $C(X)$ al conjunto de todas las funciones continuas real valuadas definidas en $X$ y por $C^{*}(X)$ al subconjunto de $C(X)$ de las funciones acotadas. También denotaremos por $f \wedge g(f \vee g)$ a la función ínfimo (a la función supremo) de $f, g$. Por $\mathcal{P}(X)$ denotaremos el conjunto potencia del conjunto $X$ y por $[X]^{\kappa}$ la colección de subconjuntos de $X$ de cardinalidad $\kappa$, mientras que $[X]^{<\kappa}$ será la colección de subconjuntos de $X$ de cardinalidad menor que $\kappa$. Para indicar que $U$ es un subconjunto abierto en $X$ que contiene a un subconjunto $A \subset X$ utilizaremos la siguiente notación: $U \in \tau(A, X)$ y si $A=\{a\}$, entonces simplemente escribiremos $U \in \tau(a, X)$ en lugar de $U \in \tau(\{a\}, X)$.

En particular, en esta sección mencionaremos algunos resultados que serán de gran importancia para el entendimiento de las secciones siguientes, se demostrarán los resultados más relevantes para fines de este trabajo.

Definición 1.1.1. Un espacio topológico $X$ es numerablemente compacto si cada cubierta abierta numerable de $X$ tiene una subcubierta finita.

Definición 1.1.2. Sea $X$ un espacio topológico. Un subespacio $D \subseteq X$ se llama discreto si para cada $d \in D$ existe $V \in \tau(d, X)$ tal que $V \cap D=\{d\}$.

Proposición 1.1.3. Un espacio topológico $T_{1}$ es numerablemente compacto si y sólo si cada subespacio cerrado y discreto es finito.

Demostración. $(\Rightarrow)$ Supongamos que $D$ es un subespacio numerablemente infinito, cerrado y discreto. Para cada $d \in D$ sean $U_{d}$ una vecindad abierta de $d$ tal que 
$U_{d} \cap D=\{d\}$ y $\mathcal{U}=\left\{U_{d}: d \in D\right\} \cup\{X \backslash D\}$, así $\mathcal{U}$ es una cubierta abierta numerable que no tiene subcubierta finita.

$(\Leftarrow)$ Sean $\mathcal{U}=\left\{U_{n}: n \in \omega\right\}$ una cubierta abierta de $X$ que no posee subcubierta finita, es decir, para cada $m \in \omega, \bigcup\left\{U_{k}: k \leq m\right\} \neq X$, así podemos escoger $x_{m} \in X \backslash \bigcup\left\{U_{k}: k \leq m\right\}$ tal que si $k \neq m$, entonces $x_{k} \neq x_{m}$ y hacemos $D=\left\{x_{n}: n \in \omega\right\}$.

Para ver que $D$ es discreto sea $x_{m} \in D$, así existe $n \in \omega$ tal que $x_{m} \in U_{n}$, entonces $V_{m}=U_{n} \backslash\left\{x_{k}: k<n\right\}$ es una vecindad de $x_{m}$ tal que $V_{m} \cap D=\left\{x_{m}\right\}$.

Para demostrar que $D$ es cerrado sea $z \in X \backslash D$. Como $\mathcal{U}$ es una cubierta abierta, existe $n \in \omega$ tal que $z \in U_{n}$. Entonces $U_{n} \backslash\left\{x_{k}: k \leq n\right\}$ es una vecindad abierta de $z$ que no intersecta a $D$.

Definición 1.1.4. Un espacio de Tychonoff $X$ es pseudocompacto si para cada $f \in$ $C(X)$, se tiene que $f[X]$ es acotado en $\mathbb{R}$, es decir, $C(X)=C^{*}(X)$.

Proposición 1.1.5. Si $X$ es un espacio de Tychonoff numerablemente compacto, entonces $X$ es pseudocompacto.

Demostración. Sea $f \rightarrow \mathbb{R}$ una función continua, entonces $f[X]$ es numerablemente compacto en $\mathbb{R}$. Como ser compacto y numerablemente compacto son propiedades equivalentes en espacios métricos, tenemos que $f[X]$ compacto, por lo tanto $f[X]$ es acotado.

El siguiente lema es el Corolario 3.10.27 de [6].

Lema 1.1.6. Si $X$ es un espacio pseudocompacto y $Y$ es un espacio compacto, entonces $X \times Y$ es pseudocompacto.

Lema 1.1.7. Sea $X$ un espacio topológico de Tychonoff y $D \subseteq X$ un subespacio denso y pseudocompacto, entonces $X$ es pseudocompacto.

Demostración. Sea $f: X \rightarrow \mathbb{R}$ una función continua. Entonces $g=f_{\left.\right|_{D}}$ es una función continua en $D$. Como $D$ es pseudocompacto, existe $M>0$ tal que $g[D] \subseteq$ $[-M, M]$, así tenemos que $f[X]=f[\operatorname{cl}(D)] \subseteq \operatorname{cl}(f[D])=\operatorname{cl}(g[D]) \subseteq \operatorname{cl}([-M, M])=$ $[-M, M]$. Por lo tanto $X$ es pseudocompacto.

Ejemplo 1.1.8. Existe un espacio que es pseudocompacto y que no es numerablemente compacto. 
Demostración. El espacio es la Plancha de Tychonoff $T=\left(\left[0, \omega_{1}\right] \times[0, \omega]\right) \backslash\left\{\left(\omega_{1}, \omega\right)\right\}$ dotado con la topología de subespacio del producto $\left[0, \omega_{1}\right] \times[0, \omega]$. El subconjunto $D=\left\{\left(\omega_{1}, n\right): n<\omega\right\}$ es cerrado y discreto en $T$. Por el Lema 1.1.3, tenemos que $T$ no es numerablemente compacto.

Falta ver que $T$ es pseudocompacto. En efecto, como $\omega_{1}$ es numerablemente compacto, por el Lema 1.1.5 tenemos que $\omega_{1}$ es pseudocompacto. Como $\omega+1$ es compacto, por el Lema 1.1.6 tenemos que el subconjunto $\omega_{1} \times(\omega+1)$ es pseudocompacto y es denso en $T$; por el Lema 1.1.7 tenemos que $T$ es pseudocompacto.

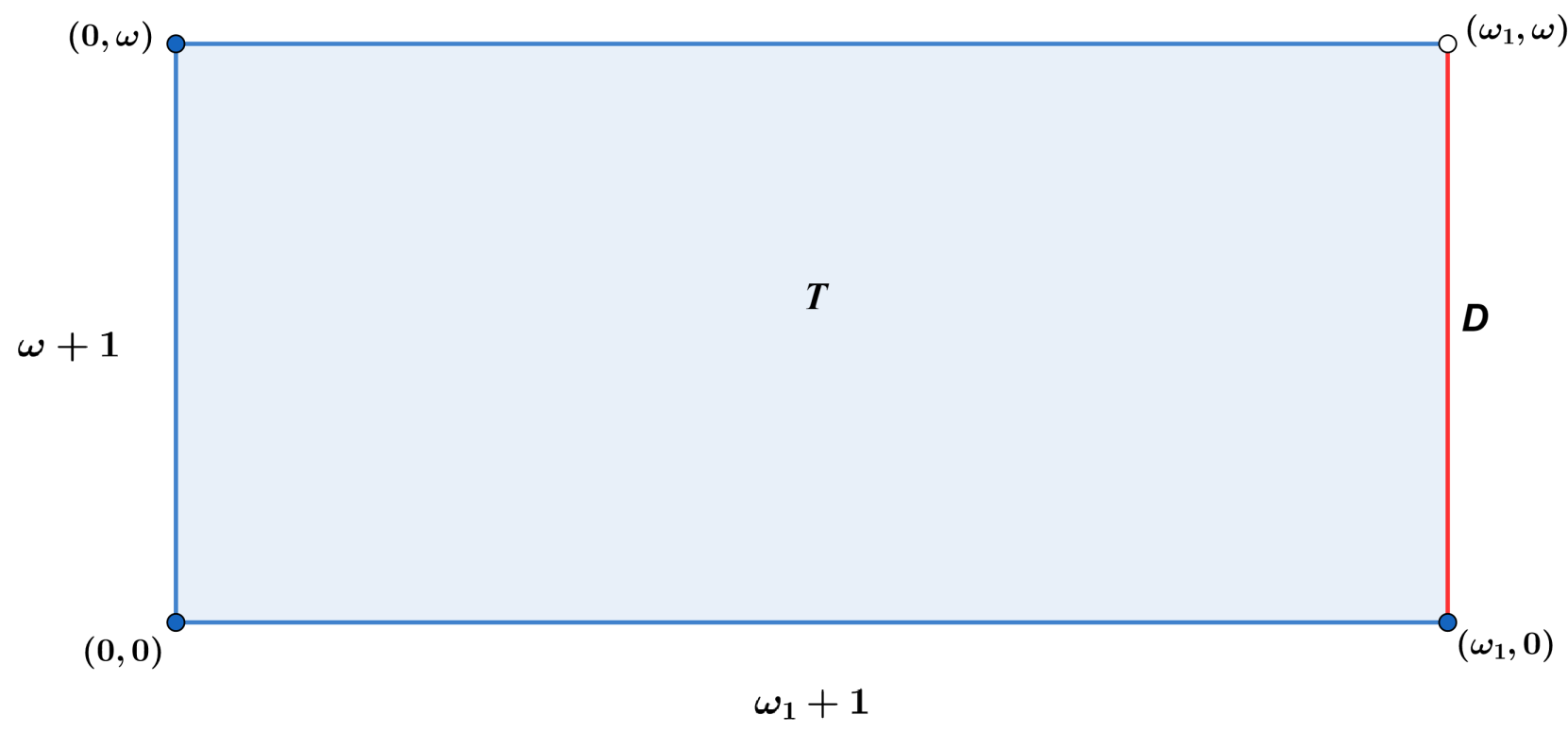

Figure 1.1: La Plancha de Tychonoff

Proposición 1.1.9. Si $X$ es un espacio pseudocompacto y $f: X \rightarrow Y$ es una función continua y sobreyectiva, entonces $Y$ es un espacio pseudocompacto. 
Demostración. Supongamos que $Y$ no es un espacio pseudocompacto, entonces existe una función $g: Y \rightarrow \mathbb{R}$ continua que no es acotada. Como $g \circ f \in C(X)$ y $(g \circ f)[X]$ no esacotado; así $X$ no es pseudocompacto.

La Plancha de Tychonoff es un ejemplo importante ya que en los siguientes capítulos mostraremos ejemplos tipo Plancha de Tychonoff. Si el lector está interesado en otras propiedades de este espacio puede consultar [19].

\subsection{Caracterizaciones de pseudocompacidad.}

Definición 1.2.1. Una familia $\mathcal{U}$ de subconjuntos en un espacio topológico $X$ se llama localmente finita si para cada $x \in X$ existe una vecindad $W$ de $x$ tal que $\mid\{U \in \mathcal{U}$ : $W \cap U \neq \emptyset\} \mid<\omega$.

Definición 1.2.2. Un espacio $X$ es tenuemente compacto si cada familia localmente finita de subconjuntos abiertos no vacíos es finita.

Lema 1.2.3. Sea $X$ un espacio topológico. Entonces las siguientes condiciones son equivalentes:

(1) $X$ es tenuemente compacto;

(2) cada familia localmente finita de subconjuntos abiertos no vacíos ajena por pares es finita;

(3) si $\left\{V_{n}: n \in \omega\right\}$ es una familia decreciente (es decir, $V_{n+1} \subseteq V_{n}$ para cada $n \in \omega$ ) de subconjuntos abiertos no vacíos de $X$, entonces $\bigcap\left\{\operatorname{cl}\left(V_{n}\right): n \in \omega\right\} \neq \emptyset$;

(4) cada cubierta abierta numerable de $X$ tiene una subfamilia finita cuya unión es densa en $X$.

Demostración. (1) $\Rightarrow$ (2) Obvio.

$(2) \Rightarrow(1)$ Supongamos que no se cumple (1), es decir, existe una familia $\left\{C_{n}: n \in \omega\right\}$ localmente finita de subconjuntos abiertos no vacíos de $X$. Sean $k_{1}=1$ y $x_{1} \in C_{k_{1}}$. Entonces podemos encontrar un subconjunto abierto $V_{1} \in \tau\left(x_{1}, X\right)$ y $k_{2} \in \omega$ tales que $V_{1} \cap C_{k}=\emptyset$ para todo $k \geq k_{2}$ y elegimos $x_{2} \in C_{k_{2}}$. Al haber elegido $x_{m-1} \in C_{k_{m-1}}$ y $V_{m-1} \in \tau\left(x_{m-1}, X\right)$ tal que $V_{m-1} \cap C_{k}=\emptyset$ para cada $k \geq k_{m}$; observemos que $\left\{V_{i}: i=1, \ldots, m-1\right\}$ es una sucesión de subconjuntos abiertos tales que $C_{k_{i}} \cap V_{i} \neq \emptyset$ y si $i<j$ entonces $V_{i} \cap C_{k_{j}}=\emptyset$. Sea $x_{m} \in C_{k_{m}}$, entonces podemos encontrar un subconjunto abierto $V_{m} \in \tau\left(x_{m}, X\right)$ y $k_{m+1} \in \omega$ tal que $V_{m} \cap C_{k}=\emptyset$ cuando $k \geq k_{m+1}$. Entonces $\left\{V_{n} \cap C_{k_{n}}: n \in \omega\right\}$ es una familia localmente finita de subconjuntos abiertos 
no vacíos ajenos dos a dos.

$(1) \Rightarrow(3)$ Supongamos que (3) es falso, sea $\left\{V_{n}: n \in \omega\right\}$ una sucesión decreciente de subconjuntos abiertos no vacíos de $X$ tal que $\bigcap\left\{\mathrm{cl}\left(V_{n}\right): n \in \omega\right\}=\emptyset$. Si $x \in X$, entonces existe $n \in \omega$ tal que si $k \geqslant n$, se tiene que $x \notin \mathrm{cl}\left(V_{k}\right)$, entonces $X \backslash \mathrm{cl}\left(V_{n}\right)$ es una vecindad abierta de $x$ que intersecta sólo a un número finito de elementos de la familia $\left\{V_{n}: n \in \omega\right\}$. Así $\left\{V_{n}: n \in \omega\right\}$ es una familia infinita localmente finita de conjuntos abiertos no vacíos de $X$.

$(3) \Rightarrow(4)$ Sea $\left\{C_{n}: n \in \omega\right\}$ una cubierta abierta de $X$ y sea $V_{m}=X \backslash \operatorname{cl}\left(\bigcup\left\{C_{j}: 1 \leqslant\right.\right.$ $j \leqslant m\})$. Entonces $\left\{V_{n}: n \in \omega\right\}$ es una sucesión decreciente de conjuntos abiertos no vacíos de $X$ y $\bigcap\left\{\operatorname{cl}\left(V_{n}\right): n \in \omega\right\} \subseteq X \backslash \bigcup\left\{C_{n}: n \in \omega\right\}=\emptyset$. Así, por hipótesis $V_{k}=\emptyset$ para algún $k \in \omega$, entonces $\bigcup\left\{C_{j}: 1 \leqslant j \leqslant k\right\}$ es densa en $X$.

$(4) \Rightarrow(2)$ Supongamos que no se cumple (2). Entonces existe una familia infinita localmente finita de conjuntos abiertos no vacíos ajenos dos a dos $\left\{V_{n}: n \in \omega\right\}$. Para cada $x \in X$ fijamos un conjunto abierto $U_{x}$ tal que $x \in U_{x}$ y tal $\left\{n \in \omega: U_{x} \cap V_{n} \neq\right.$ $\emptyset\}=F_{x}$ es un subconjunto finito de $\omega$. Sea $\mathcal{F}=[\omega]^{<\omega}$, ahora para cada $F \in \mathcal{F}$ sea $W_{F}=\bigcup\left\{U_{x}: x \in X\right.$ y $\left.F_{x}=F\right\}$. Entonces $\mathcal{C}=\left\{W_{F}: F \in \mathcal{F}\right\}$ es una cubierta abierta numerable de $X$, pues si $y \in X$ entonces existe un conjunto abierto $U_{y}$ tal que $y \in U_{y}$ y tal que $\left\{n \in \omega: U_{y} \cap V_{n} \neq \emptyset\right\}=F_{y}$, así $y \in U_{y} \subseteq W_{F_{y}}$. Sea $\left\{F_{1}, F_{2}, \ldots, F_{n}\right\}$ un subconjunto finito de $\mathcal{F}$, elegimos $k \in \omega \backslash \bigcup\left\{F_{i}: 1 \leqslant i \leqslant n\right\}$ y supongamos que $z \in\left(\bigcup\left\{W_{F_{i}}: 1 \leqslant i \leqslant n\right\}\right) \cap V_{k}$; entonces existe $j \in\{1, \ldots, n\}$ tal que $z \in W_{F_{j}} \cap V_{k}$. Entonces existe $w \in X$ tal que $z \in U_{w}$ y $F_{w}=F_{j}$. Como $k \notin F_{j}$, se sigue que $U_{w} \cap V_{k}=\emptyset$, lo cual es una contradicción. Así $\left(\bigcup\left\{W_{F_{i}}: 1 \leqslant i \leqslant n\right\}\right) \cap V_{k}=\emptyset$, por lo tanto la unión de ningún subconjunto finito de $\mathcal{C}$ es densa en $X$.

Teorema 1.2.4. Un espacio de Tychonoff $X$ es tenuemente compacto si y sólo si es pseudocompacto.

Demostración. $(\Rightarrow)$ Supongamos que $X$ es un espacio de Tychonoff tenuemente compacto y $f: X \rightarrow \mathbb{R}$ es una función continua. Para cada $n \in \omega$ sea $U_{n}=\{x \in X$ : $|f(x)|>n\}=f^{-1}[(-\infty,-n) \cup(n, \infty)]$. Ahora consideremos la familia de subconjuntos abiertos $\left\{U_{n}: n \in \omega\right\}$. Sea $x \in X$, entonces existe $m \in \omega$ tal que $|f(x)|<m$. Sea $V=f^{-1}[(f(x)-1, f(x)+1)]$, se sigue que $V$ es una vecindad de $x$ que intersecta a lo más $m$ elementos de las familia $\left\{U_{n}: n \in \omega\right\}$, por lo que la familia es localmente finita. Como $X$ es tenuemente compacto la familia es finita. Por lo tanto la función $f$ es acotada.

$(\Leftarrow)$ Supongamos que $X$ es un espacio de Tychonoff pseudocompacto y que $\mathcal{U}=\left\{U_{n}\right.$ : $n \in \omega\}$ es una familia localmente finita de subconjuntos abiertos no vacíos de $X$. Para cada $n \in \omega$ elegimos $x_{n} \in U_{n}$. Asimismo para cada $n \in \omega$ existe una función continua $f_{n}: X \rightarrow[0, n]$ tal que $f_{n}\left(x_{n}\right)=n$ y $f_{n}\left[X \backslash U_{n}\right] \subset\{0\}$. Como cada $x \in X$ está en a lo 
más un número finito de elementos de la familia $\mathcal{U}$, la función $f=\sum_{n<\omega} f_{n}$ está bien definida.

Sean $x \in X, f(x) \in(a, b)$ y $O \in \tau(x, X)$ tal que $C=\left\{n<\omega: U_{n} \cap O \neq \emptyset\right\}$ es finito. Consideremos el conjunto $A=\left\{n<\omega: f_{n}(x)>0\right\}$. Observemos que $A \subset C$ y que $f(y)=\sum_{n \in A} f_{n}(y)$ para cada $y \in O$, por lo que la función $\sum_{n \in A} f_{n}$ es continua. Se sigue que podemos encontrar una $W \in \tau(x, X)$ tal que $\sum_{n \in A} f_{n}[W] \subset(a, b)$. Sea $V=O \cap W$, entonces $x \in V$ y $f[V] \subset(a, b)$. Por lo tanto la función $f$ es continua y no acotada.

Esta equivalencia fue demostrada por Glicksberg en el año 1952. Puede consultar más información sobre la clase de los espacios pseudocompactos en [8]. Como consecuencia de este último teorema en espacios de Tychonoff podemos ocupar cualquier equivalencia del Lema 1.2.3 que caracterizan la pseudocompacidad.

Definición 1.2.5. Sea $X$ un espacio topológico; se le llama celularidad de $X$ al cardinal

$$
c(X)=\sup \{|\mathcal{U}|: \mathcal{U} \subset \tau(X) \backslash\{\emptyset\} \text { y } \mathcal{U} \text { es ajena dos a dos }\} .
$$

Si $\mathcal{F} \subseteq \tau(X)$ es una familia ajena dos a dos, diremos que $\mathcal{F}$ es una familia celular en $X$.

El siguiente lema es técnico pero es relevante para las secciones posteriores ya que lo utilizaremos un par de veces.

Lema 1.2.6. Cada familia localmente finita de subconjuntos abiertos no vacíos $\mathcal{F}$ de cardinalidad $\kappa$, tiene un refinamiento celular de cardinalidad $\kappa$.

Demostración. Sea $\mathcal{F}=\left\{F_{\alpha}: \alpha<\kappa\right\}$ una familia localmente finita de subconjuntos abiertos no vacíos. Elegimos $U_{0} \subseteq F_{0}$ tal que $U_{0}$ intersecta solamente a un número finito de elementos de $\mathcal{F}$, digamos $\mathcal{F}_{0}=\left\{F \in \mathcal{F}: F \cap U_{0} \neq \emptyset\right\}$. Al haber elegido subfamilias finitas $\mathcal{F}_{\beta}$ y subconjuntos abiertos mutuamente ajenos $U_{\beta}$ para cada $\beta<\alpha<\kappa$, notemos que $\left|\bigcup\left\{\mathcal{F}_{\beta}: \beta<\alpha\right\}\right|<|\mathcal{F}|$ y sea $\gamma=\min \left\{\xi: F_{\xi} \notin \bigcup\left\{\mathcal{F}_{\beta}: \beta<\alpha\right\}\right\}$. Como $F_{\gamma} \cap U_{\beta}=\emptyset$ para cada $\beta<\alpha$, tomamos $U_{\alpha}$ un subconjunto abierto y no vacío contenido en $F_{\gamma}$, el cual intersecta únicamente a un número finito de elementos de $\mathcal{F}$, digamos $\mathcal{F}_{\alpha}$. De esta forma la familia $\mathcal{U}=\left\{U_{\alpha}: \alpha<\kappa\right\}$ es un refinamiento celular de $\mathcal{F}$.

Corolario 1.2.7. Si $X$ es un espacio $T_{1}$ numerablemente compacto, entonces $X$ es tenuemente compacto.

Demostración. Supongamos que $\mathcal{U}=\left\{U_{n}: n \in \omega\right\}$ es una familia localmente finita de subconjuntos abiertos no vacíos de $X$, por Lema 1.2.6 $\mathcal{U}$ tiene un refinamiento celular $\mathcal{V}=\left\{V_{n}: n \in \omega\right\}$. Para cada $n \in \omega$ sea $d_{n} \in V_{n}$, se sigue que $D=\left\{d_{n}: n \in \omega\right\}$ es un subconjunto cerrado y discreto en $X$. Por lo tanto, $X$ no es numerablemente compacto. 
Lema 1.2.8. Si $X$ es un espacio y $\left\{C_{\alpha}\right\}_{\alpha<\kappa}$ es una familia localmente finita de conjuntos cerrados no vacíos, entonces $C=\bigcup_{\alpha \in \kappa} C_{\alpha}$ es cerrado.

Demostración. Sea $x \in X \backslash C$. Entonces, existe $U$ conjunto abierto que contiene $x$ tal que $F=\left\{\alpha \in \kappa: U \cap C_{\alpha} \neq \emptyset\right\}$ es un conjunto finito. Entonces el conjunto $V=U \cap\left(\bigcap_{\alpha \in F} X \backslash C_{\alpha}\right)$ es una vecindad abierta de $x$ tal que $V \cap\left(\bigcup_{\alpha \in \kappa} C_{\alpha}\right)=\emptyset$. Por lo tanto $C$ es cerrado.

Definición 1.2.9. Un espacio $X$ se llama paracompacto si cada cubierta abierta de $X$ tiene un refinamiento abierto localmente finito.

Lema 1.2.10. Si $X$ es un espacio topológico de Hausdorff y paracompacto, entonces $X$ es regular.

Demostración. Sea $D \subseteq X$ un subconjunto cerrado de $X$ y $x \in X \backslash D$. Para cada $d \in D$, existen subconjuntos ajenos $U_{d} \in \tau(x, X)$ y $V_{d} \in \tau(D, X)$. La familia $\mathcal{G}=\left\{V_{d}: d \in D\right\} \cup\{X \backslash D\}$ es una cubierta abierta de $X$; por ser $X$ paracompacto, $\mathcal{G}$ tiene un refinamiento localmente finito $\mathcal{F}$. Sea $\mathcal{U}=\{U \in \mathcal{F}: U \cap D \neq \emptyset\}$. Cada elemento de $\mathcal{U}$ es un subconjunto de un elemento de $V_{d} \in \mathcal{G}$. Como $x \notin \operatorname{cl}\left(V_{d}\right)$ para cada $d \in D, x \notin \operatorname{cl}(U)$ para cada $U \in \mathcal{U}$. Por el Lema 1.2.8, tenemos que $\bigcup\{\operatorname{cl}(U): U \in \mathcal{U}\}$ es cerrado, se sigue que $\bigcup\{U: U \in \mathcal{U}\}$ y $X \backslash \bigcup\{\operatorname{cl}(U): U \in \mathcal{U}\}$ son subconjuntos abiertos ajenos que contienen a $D$ y a $x$ respectivamente. Por lo tanto $X$ es un espacio regular.

En lo sucesivo presentaremos un espacio de gran importancia para este trabajo, ya que lo ocuparemos recurrentemente como ejemplo para demostrar que ciertas condiciones son necesarios pero no suficientes; este espacio es llamado espacio de Mrówka. Antes de definirlo requerimos los siguientes conceptos.

Definición 1.2.11. Sea $\kappa$ un cardinal. Una familia $\mathcal{A} \subset[\kappa]^{\omega}$ es una familia casi ajena $(A D)$ si para cada $A, B \in \mathcal{A}$ con $A \neq B$ se tiene que $|A \cap B|<\omega$.

Una familia $\mathrm{AD} \mathcal{A}$ es maximal casi ajena $(M A D)$ si $\mathcal{A} \subseteq \mathcal{B}$ y $\mathcal{B}$ es casi ajena implica que $\mathcal{A}=\mathcal{B}$.

Lema 1.2.12. Cada familia $\mathrm{AD}$ en $[\kappa]^{\omega}$ está contenida en una familia MAD.

Demostración. Sean $\mathcal{A}$ una familia $\mathrm{AD}$ en $[\kappa]^{\omega}$ y $\mathfrak{C}$ una cadena de familias $\mathrm{AD}$ en la familia:

$$
\mathfrak{B}=\{\mathcal{D}: \mathcal{D} \text { es una familia } \mathrm{AD} \text { y } \mathcal{A} \subseteq \mathcal{D}\}
$$

Sea $\mathcal{M}=\bigcup \mathfrak{C}$. Afirmamos que $\mathcal{M}$ es una familia $\mathrm{AD}$. Para demostrar este hecho, sean $A, B \in \mathcal{M}$, entonces existen $\mathcal{C}_{A}$ y $\mathcal{C}_{B}$ en $\mathfrak{C}$ tales que $A \in \mathcal{C}_{A}$ y $B \in \mathcal{C}_{B}$, como $\mathfrak{C}$ 
es una cadena podemos suponer sin pérdida de generalidad que $\mathcal{C}_{A} \subseteq \mathcal{C}_{B}$. Entonces $A, B \in \mathcal{C}_{B}$, ya que $\mathcal{C}_{B}$ es una familia $\mathrm{AD}$, se sigue que $|A \cap B|<\omega$. Entonces $\mathcal{M}$ es una familia $\mathrm{AD}$. Por el Lema de Zorn se sigue que $\mathfrak{C}$ tiene un elemento maximal.

Ejemplo 1.2.13. Existe una familia MAD finita en $\omega$.

Demostración. Sea $A$ el conjunto de los enteros no negativos pares y $B$ el conjunto de los enteros no negativos impares; si $C$ es cualquier conjunto infinito de $\omega$, entonces $C \cap A$ o $C \cap B$ es infinito. Por lo tanto $\{A, B\}$ es una familia MAD.

Teorema 1.2.14. Una familia MAD infinita en $[\kappa]^{\omega}$ es no numerable.

Demostración. Supongamos lo contrario, es decir, que existe una familia MAD numerablemente infinita $\mathcal{A}=\left\{A_{n}: n \in \omega\right\}$. Sea $x_{0} \in A_{0}$; al haber escogido elementos $x_{1}, \ldots, x_{n-1}$ tales que $x_{m} \in A_{m} \backslash A_{j}$ para cada $j<m<n$, consideremos el conjunto:

$$
S=A_{n} \backslash \bigcup\left\{A_{i}: 0 \leq i \leq n-1\right\}
$$

Por ser $\mathcal{A}$ una familia MAD, $S$ es infinito. Así podemos elegir $x_{n} \in S$. Sea $B=$ $\left\{x_{n}: n \in \omega\right\}$, entonces $B$ es un subconjunto infinito de $\omega$ y $B \cap A_{n}=\left\{x_{n}\right\}$, esto demuestra que $\mathcal{B}=\mathcal{A} \cup\{B\}$ es una familia $\mathrm{AD}$ y $\mathcal{A} \subsetneq \mathcal{B}$ lo que contradice que $\mathcal{A}$ es maximal.

Ejemplo 1.2.15. Existe una familia AD en $[\omega]^{\omega}$ de cardinalidad $\mathfrak{c}$.

Demostración. Para cada $r \in \mathbb{R}$ sea $\left\{x_{n}^{r}\right\}$ una sucesión no trivial de racionales que converge a $r$. Sea $\mathcal{A}=\left\{\left\{x_{n}^{r}\right\}: r \in \mathbb{R}\right\}$. Si $r, s \in \mathbb{R}$ son distintos, entonces los conjuntos $\left\{x_{n}^{r}: n \in \omega\right\}$ y $\left\{x_{n}^{s}: n \in \omega\right\}$ tienen intersección finita, ya que de lo contrario la intersección de los dos conjuntos determinaría una subsucesión de cada una de las sucesiones que convergería tanto a $r$ como a $s$. Por lo tanto $\mathcal{A}$ es una familia casi ajena de cardinalidad $\mathfrak{c}$.

Corolario 1.2.16. Existe una familia MAD $[\omega]^{\omega}$ de cardinalidad $\mathfrak{c}$.

Ahora procederemos a construir el espacio topológico prometido anteriormente. $\mathrm{Si}$ $\mathcal{A}$ es una familia MAD infinita en $[\kappa]^{\omega}$ el espacio Mrówka determinado por $\mathcal{A}$ se define como $\Psi(\mathcal{A})=\kappa \cup \mathcal{A}$ con la siguiente topología $\tau_{\Psi}$ :

(1) Si $\alpha \in \kappa$, entonces $\{\alpha\}$ es abierto.

(2) Si $A \in \mathcal{A}$, entonces una vecindad básica de $A$ es de la forma $\{A\} \cup(A \backslash F)$, donde $F \subseteq \kappa$ es finito. 
Proposición 1.2.17. Sea $\mathcal{A}$ una familia MAD infinita en $[\kappa]^{\omega} ;$ el espacio $\left(\Psi(\mathcal{A}), \tau_{\Psi}\right)$ tiene las siguientes propiedades:

(a) Es un espacio de Hausdorff;

(b) si $\kappa=\omega$ el espacio es separable;

(c) no es un espacio numerablemente compacto;

(d) es un espacio pseudocompacto.

Demostración. (a) Sean $A, B \in \mathcal{A}$ distintos. Entonces $F=A \cap B$ es finito y por tanto, $\{A\} \cup(A \backslash F)$ y $\{B\} \cup(B \backslash F)$ son vecindades ajenas de $A$ y $B$ respectivamente.

(b) El subconjunto $\omega$ es denso y numerable en $\Psi(\mathcal{A})$.

(c) Sea $x \in \Psi(\mathcal{A}) \backslash \mathcal{A}$, entonces $x \in \kappa$ y $\{x\}$ es una vecindad de $x$ que no intersecta a $\mathcal{A}$. Se sigue que $\mathcal{A}$ es un conjunto cerrado en $\Psi(\mathcal{A})$. Por otro lado, $V_{A}=\{A\} \cup A$ es una vecindad de $A$ para cada $A \in \mathcal{A}$. Más aún, $V_{A} \cap \mathcal{A}=\{A\}$, entonces $\mathcal{A}$ es un subespacio infinito, cerrado y discreto de $\Psi(\mathcal{A})$. Por el Lema 1.1.3 $\Psi(\mathcal{A})$ no es numerablemente compacto.

(d) Supongamos que $f: \Psi(\mathcal{A}) \rightarrow \mathbb{R}$ es una función continua y no acotada. Como $\kappa$ es denso en $\Psi(\mathcal{A})$ tenemos que $f$ no es acotada en $\kappa$. Entonces existe una sucesión inyectiva $\left\{x_{n}\right\}$ tal que $f\left(x_{n}\right)>n$ para cada $n \in \omega$; sea $B=\left\{x_{n}: n \in \omega\right\}$. Puesto que $\mathcal{A}$ es una familia MAD existe $A \in \mathcal{A}$ tal que $|A \cap B|=\omega$; entonces $f$ no es continua en $(A \cap B) \cup\{A\}$, pues $A \cap B$ es una sucesión que converge a $A$.

Proposición 1.2.18. Un espacio pseudocompacto y normal es numerablemente compacto.

Demostración. Supongamos que $X$ es un espacio normal pero no numerablemente compacto. Entonces por el Teorema 1.1.3, existe un subconjunto $D=\left\{d_{n}: n \in \omega\right\}$ cerrado y discreto; definimos $f: D \rightarrow \mathbb{R}$ por $f\left(d_{n}\right)=n$. Por el Teorema de Tietze, $f$ tiene una extensión continua a $X$, así hemos demostrado que $X$ no es pseudocompacto.

En el estudio de espacios topológicos y su relación con el conjunto de funciones continuas definidas en estos, es natural pensar en las propiedades que tienen las imágenes inversas y directas de dichas funciones. El conjunto $f^{-1}[\{0\}]$ será llamado el conjunto cero de la función $f: X \rightarrow \mathbb{R}$ y lo denotaremos por $Z(f)$, así que :

$$
Z(f)=\{x \in X: f(x)=0\} .
$$


$\mathrm{Al}$ complemento de un conjunto nulo lo llamaremos conjuntos cocero.

Omitiremos las demostraciones triviales de la siguiente proposición.

Proposición 1.2.19. Para un espacio topológico $X$ y dos funciones continuas $f, g$ : $X \rightarrow \mathbb{R}$ se tienen las siguientes propiedades:

(a) $Z(f)=Z(|f|)=Z\left(f^{n}\right)$ para cada $n \in \omega \backslash\{0\}$;

(b) $Z(0)=X$;

(c) $Z(f g)=Z(f) \cup Z(g)$;

(d) $Z\left(f^{2}+g^{2}\right)=Z(|f|+|g|)=Z(f) \cap Z(g)$.

Definición 1.2.20. Si $X$ es un espacio topológico, un subconjunto $G$ de $X$ se llama $G_{\delta}$ si es la intersección numerable de subconjuntos abiertos, es decir, si $G=\bigcap\left\{G_{n}: n \in \omega\right\}$ donde cada $G_{n}$ es un subconjunto abierto.

Definición 1.2.21. Si $X$ es un espacio topológico, un subconjunto $F$ de $X$ se llama $F_{\sigma}$ si es la unión numerable de subconjuntos cerrados, es decir, si $F=\bigcup\left\{F_{n}: n \in \omega\right\}$ donde cada $F_{n}$ es un subconjunto cerrado.

Proposición 1.2.22. Cada conjunto cero es un conjunto $G_{\delta}$.

Demostración. Sea $f: X \rightarrow \mathbb{R}$ una función continua, entonces:

$$
Z(f)=\bigcap_{n \in \omega}\left\{x \in X:-\frac{1}{n}<f(x)<\frac{1}{n}\right\}=\bigcap_{n \in \omega} f^{-1}\left[\left(-\frac{1}{n}, \frac{1}{n}\right)\right]
$$

Corolario 1.2.23. Cada conjunto cocero es un conjunto $F_{\sigma}$.

Para un subconjunto $A \subseteq C(X)$ tenemos que $Z[A]=\{Z(f): f \in A\}$, entonces $Z[C(X)]$ es el conjunto de todos los conjuntos cero de $X$.

Proposición 1.2.24. Si $X$ es un espacio topológico, entonces el conjunto $Z[C(X)]$ es cerrado bajo intersecciones numerables.

Demostración. Sea $f_{n} \in C(X)$ para cada $n \in \omega$ y definimos:

$$
g_{n}=\left|f_{n}\right| \wedge \frac{1}{2^{n}} \quad \text { y } g(x)=\sum_{n \in \omega} g_{n}(x)
$$


Como $\left|g_{n}(x)\right| \leq \frac{1}{2^{n}}$, por el criterio de Weierstrass la serie converge uniformemente y por lo tanto $g \in C(X)$. Entonces:

$$
Z(g)=\bigcap_{n \in \omega} Z\left(g_{n}\right)=\bigcap_{n \in \omega} Z\left(f_{n}\right) .
$$

Definición 1.2.25. Sea $X$ un espacio topológico; decimos que $G \subseteq X$ es $G_{\delta^{-}}$-denso en $X$ si para cada $A$ subconjunto $G_{\delta}$ no vacío de $X$ se cumple que $G \cap A \neq \emptyset$.

Teorema 1.2.26. Si $X$ es un espacio topológico pseudocompacto, entonces $X$ es $G_{\delta^{-}}$ denso en cada compactación de $X$.

Demostración. Sean $b X$ una compactación de $X$ y $G=\bigcap\left\{G_{n}: n \in \omega\right\}$ un subconjunto $G_{\delta}$ no vacío de $b X$, donde $G_{n}$ es un subconjunto abierto de $b X$ para cada $n \in \omega$. Supongamos que $G \cap X=\emptyset$ y sea $z \in G$; como $b X$ es un espacio de Tychonoff, para cada $n \in \omega$ existe un conjunto cero $Z_{n}$ de $b X$ tal que $z \in Z_{n} \subseteq G_{n}$.

El conjunto $Z=\bigcap\left\{Z_{n}: n \in \omega\right\}$ es un conjunto cero de $b X$ y $z \in Z \subseteq G$. Sea $f \in C(b X)$ tal que $Z=f^{-1}[\{0\}]$. Puesto que $Z(f)=Z(|f|)$ podemos suponer que $f(x) \geq 0$ para cada $x \in b X$. Así $f(x)>0$ para cada $x \in X$, como $z \in f^{-1}\left[\left[0, \frac{1}{n+1}\right)\right]$ para cada $n \in \omega$ y $X$ es denso en $b X$, entonces para cada $n \in W$ existe $x_{n} \in X$ tal que $f\left(x_{n}\right)<\frac{1}{n+1}$ por lo tanto $\left.\left(\frac{1}{f}\right)\right|_{X} \in C(X)$ y es no acotada, lo que contradice que $X$ sea pseudocompacto. 
12 CAPÍTULO 1. PSEUDOCOMPACIDAD Y COMPACIDAD NUMERABLE. 


\section{Capítulo 2}

\section{Propiedades estrella $\mathbf{P}$ y pseudocompacidad.}

\subsection{Espacios estrella P.}

En esta sección definiremos los espacios estrella $P$, donde $P$ es una propiedad topológica, y mencionaremos algunos resultados básicos sobre estos. En la primera sección nos enfocaremos en la propiedad " $P=$ finito", es decir, en los espacios estrella finita; estudiaremos la relación entre estos y los espacios topológicos con propiedades más conocidas de tipo compacidad, como la compacidad numerable y la pseudocompacidad. Además mostraremos la correspondencia que existe entre estos espacios con los espacios $n$-estrella finita y los espacios $n$-estrella numerable que primero fueron estudiados por E.K van Douwen, G.M. Roscoe y I.J. Tree en [5] y más adelante por diversos autores que iremos mencionando. Un trabajo importante sobre este tema es [16] de M. Matveev, pero en este trabajo utilizaremos la notación que fue introducida por van Mill, Tkachuk y Wilson en [3].

Definición 2.1.1. Si $X$ es un espacio topológico y $\mathcal{U}$ es una familia de subonjuntos de $X$, entonces la estrella de un conjunto $A \subseteq X$ con respecto a la familia $\mathcal{U}$ es el conjunto:

$$
S t(A, \mathcal{U})=\bigcup\{U \in \mathcal{U}: U \cap A \neq \emptyset\}
$$

Recursivamente definimos $S t^{n+1}(A, \mathcal{U})=S t\left(S t^{n}(A, \mathcal{U}), \mathcal{U}\right)$, donde $S t^{0}(A, \mathcal{U})=A$ y por tanto $S t^{1}(A, \mathcal{U})=S t(A, \mathcal{U})$.

Proposición 2.1.2. Si $X$ es un espacio topológico, $A \subseteq X$ y $\mathcal{U}$ es una cubierta abierta de $X$, entonces $\operatorname{cl}\left(S t^{n}(A, \mathcal{U})\right) \subseteq S t^{n+1}(A, \mathcal{U})$ para cada $n \in \omega$.

Demostración. Sea $z \in \operatorname{cl}\left(S t^{n}(A, \mathcal{U})\right)$, como $S t(z, \mathcal{U})$ es una vecindad abierta de z tenemos que $S t(z, \mathcal{U}) \cap S t^{n}(A, \mathcal{U}) \neq \emptyset$; se sigue que $z \in S t^{n+1}(A, \mathcal{U})$. 
Definición 2.1.3. Sea $P$ una propiedad topológica. Un espacio $X$ se dice que es estrella $P$ si para cada cubierta abierta $\mathcal{U}$ de $X$, existe un subconjunto $A \subseteq X$ que tiene la propiedad $P$ tal que $S t(A, \mathcal{U})=X$. El conjunto $A$ se llama el núcleo de la cubierta $\mathcal{U}$.

Cuando $X=S t^{n}(A, \mathcal{U})$, decimos que es estrella $P$ de orden $n$ y que $A$ es un núcleo de orden $n$.

Proposición 2.1.4. Sea $P$ una propiedad topológica. Si $X$ contiene un subespacio denso $D$ con la propiedad $P$, entonces $X$ es estrella $P$.

Demostración. Sean $\mathcal{U}$ una cubierta abierta de $X$ y $x \in X$; existe $U_{x} \in \mathcal{U}$ tal que $x \in U_{x}$ y como $D$ es denso en $X$, tenemos $U_{x} \cap D \neq \emptyset$. Así que $x \in U_{x} \subseteq S t(\mathcal{U}, D)$. Por lo que $X=S t(\mathcal{U}, D)$.

Corolario 2.1.5. Si $X$ es un espacio separable, entonces $X$ es estrella numerable.

Proposición 2.1.6. Todo espacio $T_{1}$ es estrella cerrado y discreto.

Demostración. Sean $X$ un espacio $T_{1}$ y $\mathcal{U}$ una cubierta abierta de $X$. Elegimos $x_{0} \in X$, si $S t\left(\left\{x_{0}\right\}, \mathcal{U}\right)=X$ tenemos el resultado. Si $\operatorname{St}\left(\left\{x_{0}\right\}, \mathcal{U}\right) \neq X$, entonces elegi$\operatorname{mos} x_{1} \in X \backslash S t\left(\left\{x_{0}\right\}, \mathcal{U}\right)$.

Al haber elegido $x_{\beta}$ para todo $\beta<\alpha$ tal que para cada $\beta<\alpha, x_{\beta} \notin S t\left(\left\{x_{\gamma}: \gamma<\right.\right.$ $\beta\}, \mathcal{U})$, elegimos $x_{\alpha} \in X \backslash S t\left(\left\{x_{\gamma}: \gamma<\alpha\right\}, \mathcal{U}\right)$.

Para algún $\kappa$ debemos tener que $S t\left(\left\{x_{\beta}: \beta<\kappa\right\}, \mathcal{U}\right)=X$, entonces el conjunto $D=\left\{x_{\beta}: \beta<\kappa\right\}$ es un núcleo para $\mathcal{U}$ y demostraremos que $D$ es cerrado y discreto en $X$.

Observemos que $x_{\beta} \notin S t\left(\left\{x_{\alpha}\right\}, \mathcal{U}\right)$ para cada $\beta<\alpha$ ya que si lo estuviera existiría $U \in \mathcal{U}$ tal que $x_{\beta}, x_{\alpha} \in U$ lo que implicaría que $x_{\alpha} \in S t\left(\left\{x_{\beta}\right\}, \mathcal{U}\right)$ lo que contradice la construcción. Por lo tanto $S t\left(\left\{x_{\alpha}\right\}, \mathcal{U}\right) \cap D=\left\{x_{\alpha}\right\}$, lo cual implica que $D$ es discreto.

Para ver que $D$ es cerrado, sea $z \in X \backslash D$, entonces podemos encontrar $\alpha<\kappa$ tal que $z \in S t\left(\left\{x_{\alpha}\right\}, \mathcal{U}\right)$ y sabemos que $D \cap S t\left(\left\{x_{\alpha}\right\}, \mathcal{U}\right)=\left\{x_{\alpha}\right\}$. Como $X$ es $T_{1}$ se tiene que $S t\left(\left\{x_{\alpha}\right\}, \mathcal{U}\right) \backslash\left\{x_{\alpha}\right\}$ es un conjunto abierto ajeno a $D$ que contiene a $z$; por lo que $D$ es un subconjunto cerrado.

Definición 2.1.7. Sea $X$ un espacio topológico, se le llama extensión de $X$ al cardinal

$$
e(X)=\sup \{|A|: A \text { es cerrado y discreto }\} .
$$

Corolario 2.1.8. Un espacio con extensión numerable es estrella numerable. 
Definición 2.1.9. Un espacio $X$ se dice $n$-estrella finito si para cada cubierta abierta $\mathcal{U}$ de $X$ existe un subconjunto finito $\mathcal{V}$ de $\mathcal{U}$ tal que $S t^{n}(\bigcup \mathcal{V}, \mathcal{U})=X$.

Proposición 2.1.10. Si $X$ es un espacio estrella finito de orden $n$, entonces es $n$-estrella finito.

Demostración. Sea $\mathcal{U}$ una cubierta abierta de $X$, entonces existe $F \subseteq X$ finito, $F=\left\{x_{1}, x_{2}, \ldots, x_{n}\right\}$ tal que $S t^{n}(F, \mathcal{U})=X$. Para cada $x_{i} \in F$ elegimos $U_{i} \in \mathcal{U}$ tal que $x_{i} \in U_{i}$, entonces $S t^{n}(F, \mathcal{U}) \subseteq S t^{n}\left(\bigcup_{i \leq n} U_{i}, \mathcal{U}\right)$, por lo tanto $X=S t^{n}\left(\bigcup_{i \leq n} U_{i}, \mathcal{U}\right)$.

Proposición 2.1.11. Si $X$ es un espacio estrella compacto, entonces es 1-estrella finito.

Demostración. Sea $\mathcal{U}$ una cubierta abierta de $X$. Entonces existe $F \subseteq X$ compacto, tal que $S t(F, \mathcal{U})=X$. Como $\mathcal{U}$ cubre a $F$ entonces existe un subconjunto finito $\mathcal{V}$ de $\mathcal{U}$ tal que $F \subseteq \bigcup \mathcal{V}$, por lo que $X=S t(F, \mathcal{U}) \subseteq S t(\bigcup \mathcal{V}, \mathcal{U})$.

Definición 2.1.12. Un espacio $X$ se dice n-estrella numerable, si para cada cubierta abierta $\mathcal{U}$ de $X$ existe un subconjunto numerable $\mathcal{V}$ de $\mathcal{U}$ tal que $S t^{n}(\bigcup \mathcal{V}, \mathcal{U})=X$.

Proposición 2.1.13. Si $X$ es un espacio estrella numerable, entonces es 1-estrella numerable.

Demostración. Sea $\mathcal{U}$ una cubierta abierta de $X$. Entonces existe $A \subseteq X$ numerable, $A=\left\{x_{n}: n \in \mathbb{N}\right\}$, tal que $S t(A, \mathcal{U})=X$. Para cada $n \in \omega$ elegimos $U_{n} \in \mathcal{U}$ tal que $x_{n} \in U_{n}$, entonces $S t\left(\bigcup_{n \in \omega} U_{n}, \mathcal{U}\right)=X$.

En el siguiente teorema es importante notar que no se necesita suponer axiomas de separación para el espacio $X$, en cambio para obtener la equivalencia de las dos propiedades se requiere que el espacio $X$ sea $T_{2}$.

Teorema 2.1.14. Si $X$ es numerablemente compacto, entonces es estrella finito.

Demostración. Supongamos que $X$ es numerablemente compacto y que no es estrella finito. Sea $\mathcal{U}$ una cubierta abierta tal que para todo conjunto finito $F \subseteq X$ se tiene que $S t(F, \mathcal{U}) \neq X$. Sea $x_{0} \in X$, por lo mencionado anteriormente podemos elegir $x_{1} \in X \backslash S t\left(\left\{x_{0}\right\}, \mathcal{U}\right)$. Recursivamente elegimos $x_{n} \in X \backslash S t\left(\left\{x_{0}, \ldots, x_{n-1}\right\}, \mathcal{U}\right)$, para cada $n \geq 1$. Sean $A=\left\{x_{n}: n \in \omega\right\}$ y la familia $\mathcal{V}=\left\{S t\left(\left\{x_{n}\right\}, \mathcal{U}\right): n \in \omega\right\}$.

Notemos que por la elección de $x_{n}$, cada miembro de $\mathcal{V}$ contiene exactamente un elemento de $A$, así que ningún subconjunto finito de $\mathcal{V}$ cubre a $A$.

Sea $y \in \operatorname{cl}(A)$, como $\mathcal{U}$ es cubierta de $X$ existe $U \in \mathcal{U}$ tal que $y \in U$, entonces $U \cap A \neq \emptyset$ así que $y \in \operatorname{St}\left(\left\{x_{n}\right\}, \mathcal{U}\right)$ para algún $n \in \omega$. 
Por lo tanto $\mathcal{V}$ es una cubierta abierta numerable $\operatorname{de~} \operatorname{cl}(A)$ por subconjuntos abiertos de $X$. Como $X$ es numerablemente compacto, entonces $\operatorname{cl}(A)$ también es numerablemente compacto, así que existe una subcubierta finita de $\mathcal{V}$ que cubre a $\operatorname{cl}(A)$ y por consecuencia también cubre a $A$. Esto contradice la definición de $\mathcal{V}$, pues cada elemento de $\mathcal{V}$ contiene un y sólo un elemento de $A$. Concluimos que lo supuesto es falso, así $X$ es estrella finito.

Corolario 2.1.15. Si $X$ es un espacio compacto, entonces es estrella finito.

Ejemplo 2.1.16. Existe un espacio $T_{1}$, estrella finito, que no es numerablemente compacto.

Demostración. Sea $\left(\mathbb{R}, \tau_{c}\right)$ donde $\tau_{c}$ es la topología conumerable, es decir,

$$
\tau_{c}=\{\emptyset\} \cup\{U \subseteq \mathbb{R}: \mathbb{R} \backslash U \text { es numerable }\} .
$$

Como $\{x\}=\mathbb{R} \backslash(\mathbb{R} \backslash\{x\})$, así tenemos que $\{x\}$ es cerrado para cada $x \in \mathbb{R}$, por lo que $\left(\mathbb{R}, \tau_{c}\right)$ es un espacio $T_{1}$. Además, $\left(\mathbb{R}, \tau_{c}\right)$ no es numerablemente compacto, pues $\mathbb{N}$ es un subespacio cerrado y discreto.

Para ver que $\left(\mathbb{R}, \tau_{c}\right)$ es un espacio estrella finito, sea $\mathcal{V}$ una cubierta abierta de $\left(\mathbb{R}, \tau_{c}\right)$ y elegimos un subconjunto no vacío $V \in \mathcal{V}$. Si $V=\mathbb{R}$, entonces $\{0\}$ es un núcleo finito para $\mathcal{V}$. Si $V \neq \mathbb{R}$, entonces $\mathbb{R} \backslash V$ es numerable, digamos $\mathbb{R} \backslash V=\left\{x_{n}: n \in \omega\right\}$. Para cada $n \in \omega$ sea $V_{n} \in \mathcal{V}$ tal que $x_{n} \in V_{n}$. Entonces $\mathcal{V}^{\prime}=\left\{V_{n}: n \in \omega\right\} \cup\{V\} \subseteq \mathcal{V}$ es una subcubierta de $X$. Ahora:

$$
\mathbb{R} \backslash \bigcap\left\{V_{n}: n \in \omega\right\}=\bigcup\left\{\mathbb{R} \backslash V_{n}: n \in \omega\right\}
$$

es un conjunto numerable, por lo que $\bigcap\left\{V_{n}: n \in \omega\right\}$ no es vacío. Sean $w \in \bigcap\left\{V_{n}: n \in\right.$ $\omega\}$ y $v \in V$. Entonces $S t(\{w, v\}, \mathcal{V})=S t(w, \mathcal{V}) \cup S t(v, \mathcal{V})=\left(\bigcup\left\{V_{n}: n \in \omega\right\}\right) \cup V=\mathbb{R}$; así que $\left(\mathbb{R}, \tau_{c}\right)$ es un espacio estrella finito.

Teorema 2.1.17. Si $X$ es estrella finito y de Hausdorff, entonces $X$ es numerablemente compacto.

Demostración. Supongamos que $X$ es un espacio de Hausdorff que no es numerablemente compacto. Entonces, existe un subconjunto infinito cerrado y discreto $D=\left\{x_{n}\right.$ : $n \in \omega\}$. Como $D$ es discreto, para cada $n \in \omega$, existe un subconjunto abierto $U_{n}$ tal que $U_{n} \cap D=\left\{x_{n}\right\}$.

Para cada $m \in \omega$, definimos $Y_{m}=\left\{x_{n} \in D: 2^{m} \leq n<2^{m+1}\right\}$, así $\left|Y_{m}\right|=2^{m}$. Como $Y_{m}$ es finito y $X$ es de Hausdorff, entonces existen subconjuntos abiertos ajenos $V_{n}$ tales que $x_{n} \in V_{n}$ para cada $2^{m} \leq n<2^{m+1}$. 
Definimos $\mathcal{V}_{m}=\left\{U_{n} \cap V_{n}: 2^{m} \leq n<2^{m+1}\right\}$ que es una colección de subconjuntos abiertos ajenos, tales que $\left(U_{n} \cap V_{n}\right) \cap D=\left\{x_{n}\right\}$. Sea $\mathcal{V}=\{X \backslash D\} \cup \bigcup\left\{\mathcal{V}_{m}: m \in \omega\right\}$. Así tenemos que $\mathcal{V}$ es una cubierta abierta numerable de $X$.

Sea $A$ un subconjunto finito de $X$, con $|A|=M$. Entonces $|A|<2^{M}=\left|\mathcal{V}_{M}\right|$. Así, para algún $m$ tal que $2^{M} \leq m \leq 2^{M+1}$ tenemos que $\left(U_{m} \cap V_{m}\right) \cap A=\emptyset$. Pero $U_{m} \cap V_{m}$ es el único elemento de $\mathcal{V}$ que contiene a $x_{m}$, así $x_{m} \notin S t(A, \mathcal{V})$, es decir, $S t(A, \mathcal{V}) \neq X$. Como $A$ se eligió arbitrariamente, se tiene que $X$ no es estrella finito.

Ejemplo 2.1.18. Existe un espacio de Hausdorff 1-estrella finito, segundo numerable que no es estrella finito.

Demostración. Sean $Y=\bigcup\{[0,1] \times\{n\}: n \in \omega\}$ y $X=Y \cup\{a\}$, donde $a \notin Y$. Definimos una base para la topología en $X$ de la siguiente manera:

i) $[0,1] \times \mathbb{N}$ tiene la topología producto.

ii) Un conjunto abierto básico que contiene a $a$ es de la forma $\{a\} \cup \bigcup\{[0,1) \times\{n\}$ : $n>m\}$ con $m \in \omega$.

Para demostrar que esta topología es de Hausdorff, sean $z_{1}, z_{2} \in X$ distintos:

1) Si $z_{1}, z_{2} \in Y$, entonces $z_{1} \in[0,1] \times\left\{n_{1}\right\}=V_{1}$ y $z_{2} \in[0,1] \times\left\{n_{2}\right\}=V_{2}$, tales que $n_{1}, n_{2} \in \omega$. Si $n_{1} \neq n_{2}$, entonces $V_{1}$ y $V_{2}$ son dos subconjuntos abiertos ajenos que separan a dichos puntos. Si $n_{1}=n_{2}$, sea $r=\left|z_{1}-z_{2}\right|$ y tomamos $\epsilon=\frac{r}{2}$, así las vecindades $W_{1}=\left(z_{1}-\epsilon, z_{1}+\epsilon\right) \times\left\{n_{1}\right\}$ y $W_{2}=\left(z_{2}-\epsilon, z_{2}+\epsilon\right) \times\left\{n_{1}\right\}$ son vecindades ajenas de $z_{1}$ y $z_{2}$ respectivamente.

2) Si $z_{1} \in Y$ y $z_{2}=a$, entonces $z_{1} \in[0,1] \times\left\{n_{1}\right\}=V_{1}$, con $n_{1} \in \omega$, por lo que basta tomar $U_{a}=\{a\} \cup\left\{[0,1) \times\{n\}: n>n_{1}\right\}$, así $U_{a} \cap V_{1}$ son vecindades ajenas de $a$ y $z_{1}$ respectivamente.

Para ver que este espacio no es estrella finito, basta fijarnos en el subconjunto $B=$ $\{(1, n): n \in \omega\}$ que es un subconjunto infinito, cerrado y discreto en $X$. Por lo tanto $X$ no es numerablemente compacto y por el Teorema 2.1 .17 no es estrella finito.

Para ver que $X$ es un espacio 1 -estrella finito. Sea $\mathcal{U}$ una cubierta por subconjuntos abiertos básicos de $X$, elegimos $W \in \mathcal{U}$ tal que $a \in W$, entonces $W=\{a\} \cup$ $\bigcup\{[0,1) \times\{n\}: n>m\}$ para algún $m \in \omega$. Notemos que $\bigcup\{[0,1] \times\{n\}: n \leq m\}$ es compacto, pues es la unión finita de subespacios compactos, por lo que podemos encontrar un subconjunto finito $\mathcal{U}^{\prime} \subset \mathcal{U}$ tal que $\bigcup\{[0,1] \times\{n\}: n \leq m\} \subseteq \cup \mathcal{U}^{\prime}$, haciendo $\mathcal{V}=\mathcal{U}^{\prime} \cup\{W\}$ tenemos que $S t(\bigcup \mathcal{V}, \mathcal{U})=X$; pues $\bigcup \mathcal{V}$ es denso en $X$. 


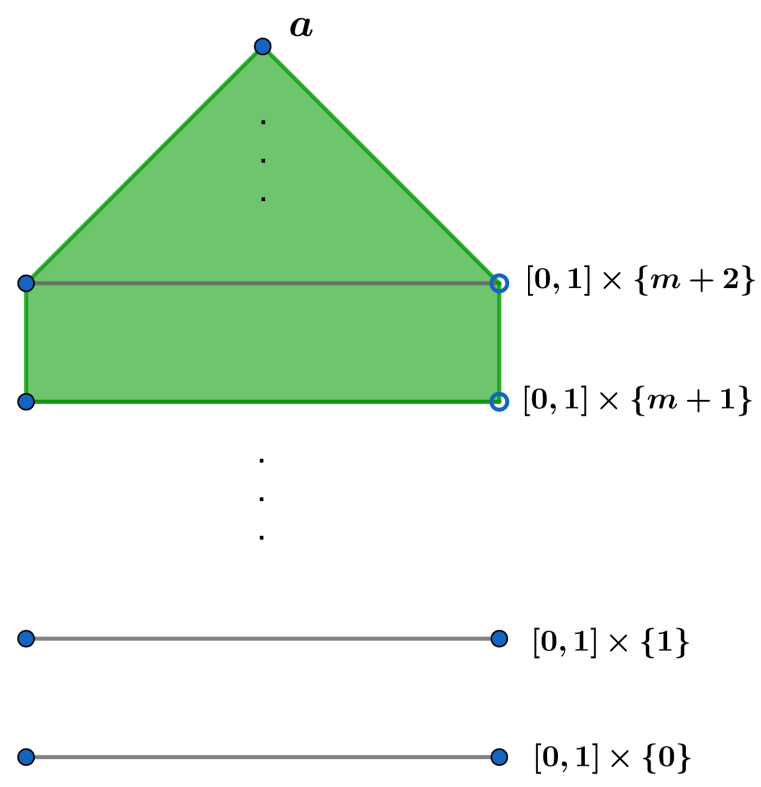

Figure 2.1: Espacio 1-estrella finito que no es estrella finito

Lema 2.1.19. Si $(X, \tau)$ es un espacio $T_{3}$ y $D=\left\{d_{n}: n \in \omega\right\} \subseteq X$ es un subespacio discreto, entonces existe $\left\{U_{n}: n \in \omega\right\} \subseteq \tau$ tal que $U_{n} \cap D=\left\{d_{n}\right\}$ para cada $n \in \omega$ y $U_{n} \cap U_{m}=\emptyset$ si $n \neq m$.

Demostración. Como $X$ es un espacio $T_{3}$, para cada $n \in \omega$ existen subconjuntos ajenos $V_{n} \in \tau\left(d_{n}, X\right)$ y $W_{n} \in \tau\left(\operatorname{cl}(D) \backslash\left\{d_{n}\right\}, X\right)$. Haciendo $U_{0}=V_{0}$ y para cada $n \in \omega U_{n}=V_{n} \cap\left(\bigcap\left\{W_{m}: m<n\right\}\right)$ tenemos que la familia $\left\{U_{n}: n \in \omega\right\}$ cumple las condiciones buscadas.

A continuación presentaremos un ejemplo de un espacio 1-estrella finito que no es estrella compacto, para ello necesitamos la construcción del siguiente espacio:

Ejemplo 2.1.20. El producto de dos espacios numerablemente compactos no es necesariamente numerablemente compacto.

Demostración. Consideremos a $\beta \omega$ la compactación de Stone-Čech de $\omega$. Para cada $M \subseteq \beta \omega$ nos fijamos en $[M]^{\omega}$; sea $f$ la función que asigna a cada elemento $A$ de $[\beta \omega]^{\omega}$ un punto de acumulación del conjunto $A$ en el espacio $\beta \omega$. Sea $X_{0}=\omega \mathrm{y}$

$$
X_{\alpha}=\left(\bigcup\left\{X_{\gamma}: \gamma<\alpha\right\}\right) \cup f\left[\left[\bigcup\left\{X_{\gamma}: \gamma<\alpha\right\}\right]^{\omega}\right] \text { para } 0<\alpha<\omega_{1} .
$$


Por inducción definimos la sucesión $\left\{X_{\alpha}: \alpha<\omega_{1}\right\}$ de subconjuntos de $\beta \omega$. El espacio $X=\bigcup\left\{X_{\alpha}: \alpha<\omega_{1}\right\}$ es numerablemente compacto, ya que si $C$ es un subconjunto infinito numerable de $X$, entonces existe $\beta<\omega_{1}$ tal que $C \subset X_{\beta}$ por lo que $C$ tiene un punto de acumulación en $X_{\beta+1}$ y por lo tanto en $X$. Observemos que si $X_{\alpha}$ tiene cardinalidad a lo más $\mathfrak{c}$, entonces hay $\mathfrak{c}^{\omega}=\mathfrak{c}$ subconjuntos de cardinalidad $\omega$ de $X_{\alpha}$ y por lo tanto $X_{\alpha+1}$ también tiene cardinalidad a lo más c. Además, si $\alpha$ es un ordinal límite y $X_{\beta}$ tiene cardinalidad $\mathfrak{c}$ para cada $\beta<\alpha$, entonces $\bigcup\left\{X_{\beta}: \beta<\alpha\right\}$ tiene cardinalidad $\omega \cdot \mathfrak{c}=\mathfrak{c}$ y otra vez como $\bigcup\left\{X_{\beta}: \beta<\alpha\right\}$ tiene a lo más $\mathfrak{c}$ subconjuntos de cardinalidad $\omega$ se sigue que $X_{\alpha}$ tiene cardinalidad $\mathfrak{c}$. Finalmente como $X=\bigcup\left\{X_{\beta}: \beta<\omega_{1}\right\}$ y cada $X_{\beta}$ tiene cardinalidad a lo mas $\mathfrak{c}$, se sigue que $|X| \leq \omega_{1} \cdot \mathfrak{c}=\mathfrak{c}$. El subespacio $Y=\omega \cup(\beta \omega \backslash X)$ es numerablemente compacto, ya que $|\operatorname{cl}(A)|=2^{\mathfrak{c}}$ para cada $A \in[\beta \omega]^{\omega}$. Si $B \subset Y$ es un subconjunto numerablemente infinito, tenemos que $B$ tiene $2^{\mathfrak{c}}$ puntos de acumulación en $\beta \omega$ de los cuales hay a lo más $\mathfrak{c}$ en $X$, por lo que existe $p \in Y$ tal que $p$ es punto de acumulación de $B$.

Ahora consideremos el producto cartesiano $X \times Y$ y sea $\triangle_{0}=(X \times Y) \cap \triangle$, donde $\triangle=\{(\delta, \delta): \delta \in \beta \omega\}$. Como $X \cap Y=\omega$, tenemos que $\triangle_{0}=\{(n, n): n \in \omega\}$. Ya que $\{n\}$ es un subconjunto abierto en $\beta \omega$ para cada $n \in \omega$, tenemos que $\triangle_{0}$ es un subconjunto abierto y discreto en $X \times Y$. Como $\triangle$ es un subconjunto cerrado en $\beta \omega$ tenemos que $\triangle_{0}$ es cerrado en $X \times Y$, por lo tanto el espacio producto no es numerablemente compacto.

El siguiente resultado es la Proposición 3.5 de [24].

Proposición 2.1.21. Cada subespacio numerable de $\beta \omega$ es $C^{*}$-inmerso.

Corolario 2.1.22. Sean $C, D$ dos subespacios discretos numerablemente infinitos y ajenos de $\beta \omega$, entonces $\operatorname{cl}(C) \cap \operatorname{cl}(D)=\emptyset$.

Demostración. Sea $f: C \cup D \rightarrow[0,1]$, tal que:

$$
f(x)=\left\{\begin{array}{lll}
0, & \text { si } & x \in C, \\
1, & \text { si } & x \in D .
\end{array}\right.
$$

Por la Proposición 2.1.21, existe una extensión continua $g$ de $f$, así $g[\operatorname{cl}(C)] \subseteq$ $\operatorname{cl}(g[C])=\{0\}$ y $g[\operatorname{cl}(D)] \subseteq \operatorname{cl}(g[D])=\{1\}$.

Ejemplo 2.1.23. Existe un espacio 1-estrella finito y estrella numerablemente compacto que no es estrella compacto.

Demostración. Consideremos el espacio $\omega^{*}=\beta \omega \backslash \omega$ y sean $X, Y$ los subespacios numerablemente compactos de $\beta \omega$ construidos en el ejemplo anterior, $G=(X \backslash \omega) \subset \omega^{*}$ y $H=\omega^{*} \backslash G$. Notemos que cada subconjunto compacto en $G$ y en $H$ es finito, pues 
cada subconjunto infinito de $G$ tiene puntos de acumulación en $H$ y viceversa.

Sean $D \subset G$ un subespacio numerablemente infinito y discreto, y $Z=G \backslash\left(\operatorname{cl}_{\omega^{*}}(D) \backslash D\right)$. Tenemos que $D$ es un subespacio cerrado y discreto de $Z$, ya que $D^{d}=\emptyset$ y por lo tanto $Z$ no es numerablemente compacto. Supongamos que $Z$ es estrella compacto, entonces para cada cubierta abierta $\mathcal{U}$ de $Z$, existe un subconjunto compacto $K$ tal que $S t(K, \mathcal{U})=Z$. Como todos los subconjuntos compactos de $Z$ son finitos, tenemos que $Z$ es estrella finito y por el Teorema 2.1 .17 podríamos concluir que $Z$ es numerablemente compacto, lo que es una contradicción.

Probaremos simultáneamente que $Z$ es estrella numerablemente compacto y 1-estrella finito; para esto sea $\mathcal{U}$ una cubierta abierta de $Z$. Para cada $z \in Z \backslash D$ elegimos $U_{z} \in \mathcal{U}$ tal que $z \in U_{z}$, como $\omega^{*}$ es un espacio regular podemos encontrar $W_{z} \in \tau\left(z, \omega^{*}\right)$ tal que $\mathrm{cl}_{\omega^{*}}\left(W_{z}\right) \cap \mathrm{cl}_{\omega^{*}}(D)=\emptyset$, por lo que existe un $U_{z}^{\prime} \subset U_{z}$ que contiene a $z \mathrm{y}$ $\operatorname{cl}_{\omega^{*}}\left(U_{z}^{\prime}\right) \cap \operatorname{cl}_{\omega^{*}}(D)=\emptyset$, hacemos $\mathcal{U}_{0}=\left\{U_{z}^{\prime}: z \in Z \backslash D\right\}$. Para cada $d \in D$ elegimos $U_{d} \in \mathcal{U}$ tal que $d \in U_{d}$ y podemos asegurar que $U_{d} \cap\left(\operatorname{cl}_{\omega^{*}}(D) \backslash D\right)=\emptyset$, ya que $\operatorname{cl}_{\omega^{*}}(D) \backslash D$ es un subconjunto cerrado; por el Lema 2.1.19 podemos encontrar una familia de subconjuntos abiertos ajenos dos a dos $\left\{V_{d}: d \in D\right\}$ tales que $V_{d} \cap D=\{d\}$ para cada $d \in D$. Sean $W_{d}=U_{d} \cap V_{d}$ para cada $d \in D$ y $\mathcal{U}_{1}=\left\{W_{d}: d \in D\right\}$, así tenemos que la familia $\mathcal{U}_{0} \cup \mathcal{U}_{1}$ es un refinamiento abierto de la familia $\mathcal{U}$. Para obtener el resultado basta encontrar un núcleo numerablemente compacto para $\mathcal{U}_{0} \cup \mathcal{U}_{1}$ y una subfamilia finita $\mathcal{V}$ de $\mathcal{U}_{0} \cup \mathcal{U}_{1}$ tal que $S t\left(\cup \mathcal{V}, \mathcal{U}_{0} \cup \mathcal{U}_{1}\right)=Z$. Sean $O(U) \in \tau\left(\omega^{*}\right)$ tal que $O(U) \cap Z=U$ para cada $U \in \mathcal{U}_{0} \cup \mathcal{U}_{1}$ y $\mathcal{O}=\left\{O(U): U \in \mathcal{U}_{0} \cup \mathcal{U}_{1}\right\}$. Como el subconjunto $M=\omega^{*} \backslash(\bigcup \mathcal{O})$ es cerrado, entonces es compacto y notemos que el subconjunto $F=\left(\operatorname{cl}_{\omega^{*}}(D) \backslash D\right) \subset M$. Afirmamos que $M \backslash F$ es finito. En efecto, si esto no fuera cierto, entonces podríamos encontrar un subconjunto infinito y discreto $C \subset M \backslash F$. Como el subespacio $D \cup C$ es discreto, por el Corolario 2.1.22 concluimos que $\operatorname{cl}(D) \cap \operatorname{cl}(C)=\emptyset$ y por lo tanto $\operatorname{cl}(C)$ es un subconjunto infinito y compacto de $H$, lo que es una contradicción.

Sea $M \backslash F=\left\{p_{1}, \ldots, p_{n}\right\}$, como $p_{i} \notin \operatorname{cl}(D)$ existen vecindades ajenas $U_{i} \in \tau\left(p_{i}, \omega^{*}\right)$ y $V_{i} \in \tau\left(\operatorname{cl}(D), \omega^{*}\right)$ para cada $1 \leq i \leq n$. Entonces $W=\bigcup\left\{U_{i}: 1 \leq i \leq n\right\}$ y $\bigcap\left\{V_{i}: 1 \leq i \leq n\right\}$ son vecindades ajenas de $M \backslash F$ y de $\operatorname{cl}(D)$ respectivamente. Notemos que $U_{d} \backslash W \neq \emptyset$ para cada $d \in D$, ya que $d \in U_{d} \backslash W$. Elegimos $x_{d} \in U_{d} \backslash(W \cup\{d\})$. Entonces $\operatorname{cl}\left(\left\{x_{d}: d \in D\right\}\right) \cap(M \backslash F)=\emptyset$, el conjunto $D \cup\left\{x_{d}: d \in D\right\}$ es discreto, $D \cap\left\{x_{d}: d \in D\right\}=\emptyset$ y por el Corolario $2.1 .22 \operatorname{cl}(D) \cap \operatorname{cl}\left(\left\{x_{d}: d \in D\right\}\right)=\emptyset$. Puesto que $N=\operatorname{cl}_{\omega^{*}}\left(\left\{x_{d}: d \in D\right\}\right)$ es ajeno a $M$, se sigue que $N$ es un subconjunto compacto de $\omega^{*} \backslash M$ y por lo tanto está cubierto por $\mathcal{O}$. Entonces podemos encontrar una subfamilia finita $\mathcal{U}^{\prime} \subset \mathcal{U}_{0} \cup \mathcal{U}_{1}$ tal que $N \subseteq \cup \mathcal{U}^{\prime}$. Ya que $N$ intersecta a todos los elementos de $\mathcal{U}_{1}$, entonces $S t\left(\bigcup \mathcal{U}^{\prime}, \mathcal{U}_{0} \cup \mathcal{U}_{1}\right) \supset \bigcup \mathcal{U}_{1}$. Notemos que también el conjunto $K=\operatorname{cl}\left(\left\{x_{d}: d \in D\right\}\right) \cap Z$ es numerablemente compacto y $S t\left(K, \mathcal{U}_{0} \cup \mathcal{U}_{1}\right) \supseteq \bigcup \mathcal{U}_{1}$.

Si $S \subset Z \backslash D$ es un subconjunto numerablemente infinito como $\operatorname{cl}_{\omega^{*}}(S) \cap \operatorname{cl}_{\omega^{*}}(D)=\emptyset$, 
entonces $S$ tiene un punto de acumulación en $G$ y por lo tanto en $Z \backslash D$, se sigue que el espacio $Z^{\prime}=Z \backslash D$ es numerablemente compacto; por lo que existe un subconjunto finito $E \subset Z^{\prime}$ tal que $S t\left(E, \mathcal{U}_{0} \cup \mathcal{U}_{1}\right) \supseteq Z \backslash \operatorname{cl}(D)$. Por lo tanto $L=E \cup K$ es un subespacio numerablemente compacto tal que $S t\left(L, \mathcal{U}_{0} \cup \mathcal{U}_{1}\right)=Z$, así concluimos que el espacio es estrella numerablemente compacto. Si elegimos una subfamilia finita $\mathcal{V} \subseteq \mathcal{U}_{0} \cup \mathcal{U}_{1}$ tal que $E \subset \cup \mathcal{V}$, entonces $\mathcal{W}=\mathcal{U}^{\prime} \cup \mathcal{V}$ es una subfamilia finita de $\mathcal{U}_{0} \cup \mathcal{U}_{1}$ tal que $S t\left(\cup \mathcal{W}, \mathcal{U}_{0} \cup \mathcal{U}_{1}\right)=Z$, se sigue que $Z$ es 1-estrella finito.

\subsection{Relaciones entre espacios pseudocompactos $y$ espacios estrella $P$.}

En esta sección mostraremos la relación entre los espacios estrella $P$ y los espacios pseudocompactos, notemos que en espacios $T_{2}$ la propiedad de ser estrella finito implica compacidad tenue como consecuencia del Teorema 2.1.17.

Definición 2.2.1. Decimos que un espacio topológico $X$ es estrella $P$ de orden $\omega$ si para cada cubierta abierta $\mathcal{U}$ de $X$ existe algún $n \in \omega$ y un subconjunto $A$ de $X$ con la propiedad $P$ tal que $S t^{n}(A, \mathcal{U})=X$.

Definición 2.2.2. Decimos que un espacio topológico $X$ es $\omega$-estrella finito si para cada cubierta abierta $\mathcal{U}$ de $X$ existe algún $n \in \omega$ y subfamilia finita $\mathcal{V} \subseteq \mathcal{U}$ tal que $S t^{n}(\bigcup \mathcal{V}, \mathcal{U})=X$.

Lema 2.2.3. Si $X$ es un espacio estrella finito de orden $\omega$, entonces $X$ es $\omega$-estrella finito.

Demostración. Sea $\mathcal{U}$ una cubierta abierta de $X$. Entonces existe $n \in \omega$ y un subconjunto finito $A$ de $X$ tal que $S t^{n}(A, \mathcal{U})=X$, para cada $x \in A$ sea $U_{x} \in \mathcal{U}$ tal que $x \in U_{x}$. Sea $\mathcal{V}=\left\{U_{x}: x \in A\right\} ;$ así $X=S t^{n}(A, \mathcal{U}) \subseteq S t^{n}(\bigcup \mathcal{V}, \mathcal{U})$.

Estas propiedades son más débiles que la compacidad numerable; en efecto, la clase de los espacios de Tychonoff con estas propiedades yacen entre la clase de los espacios numerablemente compactos y la de los espacios pseudocompactos.

Teorema 2.2.4. Si $X$ es un espacio de Tychonoff y estrella finito de orden $\omega$, entonces $X$ es pseudocompacto.

Demostración. Sea $f: X \rightarrow \mathbb{R}$ una función continua. Definimos $\mathcal{U}=\left\{f^{-1}[(k, k+2)]\right.$ : $k \in \mathbb{Z}\}$. Entonces $\mathcal{U}$ es una cubierta abierta de $X$. Por el Lema 2.2.3 se tiene que existen $n \in \omega$ y una subfamilia finita $\mathcal{V} \subseteq \mathcal{U}$ tal que $S t^{n}(\bigcup \mathcal{V}, \mathcal{U})=X$. Sea $M=\max \left\{k+2: f^{-1}[(k, k+2)] \in \mathcal{V}\right\}$ y $m=\min \left\{k: f^{-1}[(k, k+2)] \in \mathcal{V}\right\}$. 
Notemos que para cada $f^{-1}[(k, k+2)]$, se tiene que $S t\left(f^{-1}[(k, k+2)], \mathcal{U}\right) \subseteq f^{-1}[(k-$ $1, k+3)]$. Sea $x \in X$, entonces para $1 \leq j \leq n$ existe $f^{-1}\left[\left(k_{j}, k_{j}+2\right)\right] \in \mathcal{U}$ tal que $x \in f^{-1}\left[\left(k_{n}, k_{n}+2\right)\right]$ con $f^{-1}\left[\left(k_{j}, k_{j}+2\right)\right] \cap f^{-1}\left[\left(k_{j+1}, k_{j+1}+2\right)\right] \neq \emptyset$ y $f^{-1}\left[\left(k_{1}, k_{1}+\right.\right.$ $2)] \cap(\bigcup \mathcal{V}) \neq \emptyset$. Por construcción, $f(\bigcup \mathcal{V}) \subseteq(m, M)$, inductivamente podemos ver que $f(x) \in(m-n, M+n)$, lo que muestra que $f$ es una función acotada, por lo tanto $X$ es un espacio pseudocompacto.

Definición 2.2.5. Una familia $\mathcal{U}$ de subconjuntos de un espacio topológico $X$ se llama discreta si para cada $x \in X$ existe una vecindad $W$ de $x$ tal que $\mid\{U \in \mathcal{U}: W \cap U \neq$ $\emptyset\} \mid \leq 1$.

Teorema 2.2.6. Si $X$ es un espacio topológico tal que cada familia discreta de subconjuntos abiertos no vacíos es finita, entonces $X$ es 2 -estrella finito.

Demostración. Supongamos que $X$ no es 2-estrella finito y sea $\mathcal{U}$ una cubierta abierta de $X$ tal que para cada subfamilia finita $\mathcal{V}$ de $\mathcal{U}$ se tiene que $S t^{2}(\bigcup \mathcal{V}, \mathcal{U}) \neq X$.

Elegimos $U_{0} \in \mathcal{U}$ y definimos $\mathcal{V}_{0}=\left\{U_{0}\right\}$. Supongamos que para cada $0 \leq k<n$ hemos definido una familia discreta de $k$ elementos de $\mathcal{U}$ tal que $\mathcal{V}_{k-1} \subseteq \mathcal{V}_{k}$. Así podemos elegir $x_{n} \in X \backslash S t^{2}\left(\bigcup \mathcal{V}_{n-1}, \mathcal{U}\right)$ y $U_{n} \in \mathcal{U}$ tal que $x_{n} \in U_{n}$. Sea $\mathcal{V}_{n}=\mathcal{V}_{n-1} \cup\left\{U_{n}\right\}$; veamos que $\mathcal{V}_{n}$ es una familia discreta. Con este fin, supongamos que existen $y \in X$ y $l<m \leq n$ tales que $U_{l}, U_{m} \in \mathcal{V}_{n}$ con la siguiente condición $V \cap U_{l} \neq \emptyset \neq V \cap U_{m}$ para cada vecindad $V$ de $y$.

Sea $U_{y} \in \mathcal{U}$ tal que $y \in U_{y}$, como $U_{y} \cap U_{l} \neq \emptyset$ tenemos que $U_{y} \subseteq \operatorname{St}\left(\bigcup \mathcal{V}_{l}, \mathcal{U}\right)$. Ahora, como $U_{y} \cap U_{m} \neq \emptyset$ concluimos que $U_{m} \subseteq S t^{2}\left(\bigcup \mathcal{V}_{l}, \mathcal{U}\right)$. Entonces $x_{m} \in U_{m} \subseteq$ $S t^{2}\left(\bigcup \mathcal{V}_{l}, \mathcal{U}\right) \subseteq S t^{2}\left(\bigcup \mathcal{V}_{m-1}, \mathcal{U}\right)$, esto contradice la elección de $x_{m}$. Así para cada $y \in X$ existe un conjunto abierto que lo contiene y que intersecta a lo más a un elemento de $\mathcal{V}_{n}$.

Definiendo $\mathcal{V}=\bigcup\left\{\mathcal{V}_{n}: n \in \omega\right\}$, un argumento idéntico muestra que $\mathcal{V}$ es una familia numerable, infinita y discreta de subconjuntos abiertos.

Corolario 2.2.7. Cada espacio tenuemente compacto es 2-estrella finito.

Ejemplo 2.2.8. Existe un espacio de Tychonoff pseudocompacto (por lo tanto tenuemente compacto) que no es 1-estrella numerable.

Demostración. Sean $D=\left\{d_{\alpha}: \alpha<\omega_{1}\right\}$ un espacio discreto y $X=\left(\beta D \times\left(\omega_{1}+1\right)\right) \backslash$ $\left((\beta D \backslash D) \times\left\{\omega_{1}\right\}\right)$ un subespacio de $\beta D \times\left(\omega_{1}+1\right) ; X$ es un espacio pseudocompacto, ya que $\beta D \times \omega_{1}$ es un subconjunto denso numerablemente compacto de $X$. Por la Proposición 2.1.4 tenemos que $X$ es estrella numerablemente compacto. 
Mostremos que $X$ no es 1-estrella numerable. Para cada $\alpha<\omega_{1}$, sean $U_{\alpha}=\left\{d_{\alpha}\right\} \times$ $\left(\alpha, \omega_{1}\right]$ y $V_{\alpha}=\beta D \times[0, \alpha)$. Consideremos la cubierta abierta:

$$
\mathcal{U}=\left\{U_{\alpha}: \alpha<\omega_{1}\right\} \cup\left\{V_{\alpha}: \alpha<\omega_{1}\right\}
$$

Sea $\mathcal{V}$ un subconjunto numerable de $\mathcal{U}$, entonces existen $\alpha_{1}, \alpha_{2}<\omega_{1}$ tales que $U_{\alpha} \notin \mathcal{V}$ para cada $\alpha>\alpha_{1}$ y $V_{\alpha} \notin \mathcal{V}$ para cada $\alpha>\alpha_{2}$. Si tomamos $\alpha^{\prime}>\max \left\{\alpha_{1}, \alpha_{2}\right\}$, entonces el punto $\left(d_{\alpha^{\prime}}, \omega_{1}\right) \notin S t(\bigcup \mathcal{V}, \mathcal{U})$, ya que $U_{\alpha^{\prime}}$ es el único elemento de $\mathcal{U}$ que contiene a $\left(d_{\alpha^{\prime}}, \omega_{1}\right)$ y $U_{\alpha^{\prime}} \cap(\bigcup \mathcal{V})=\emptyset$, lo cual demuestra que $X$ no es 1-estrella numerable.

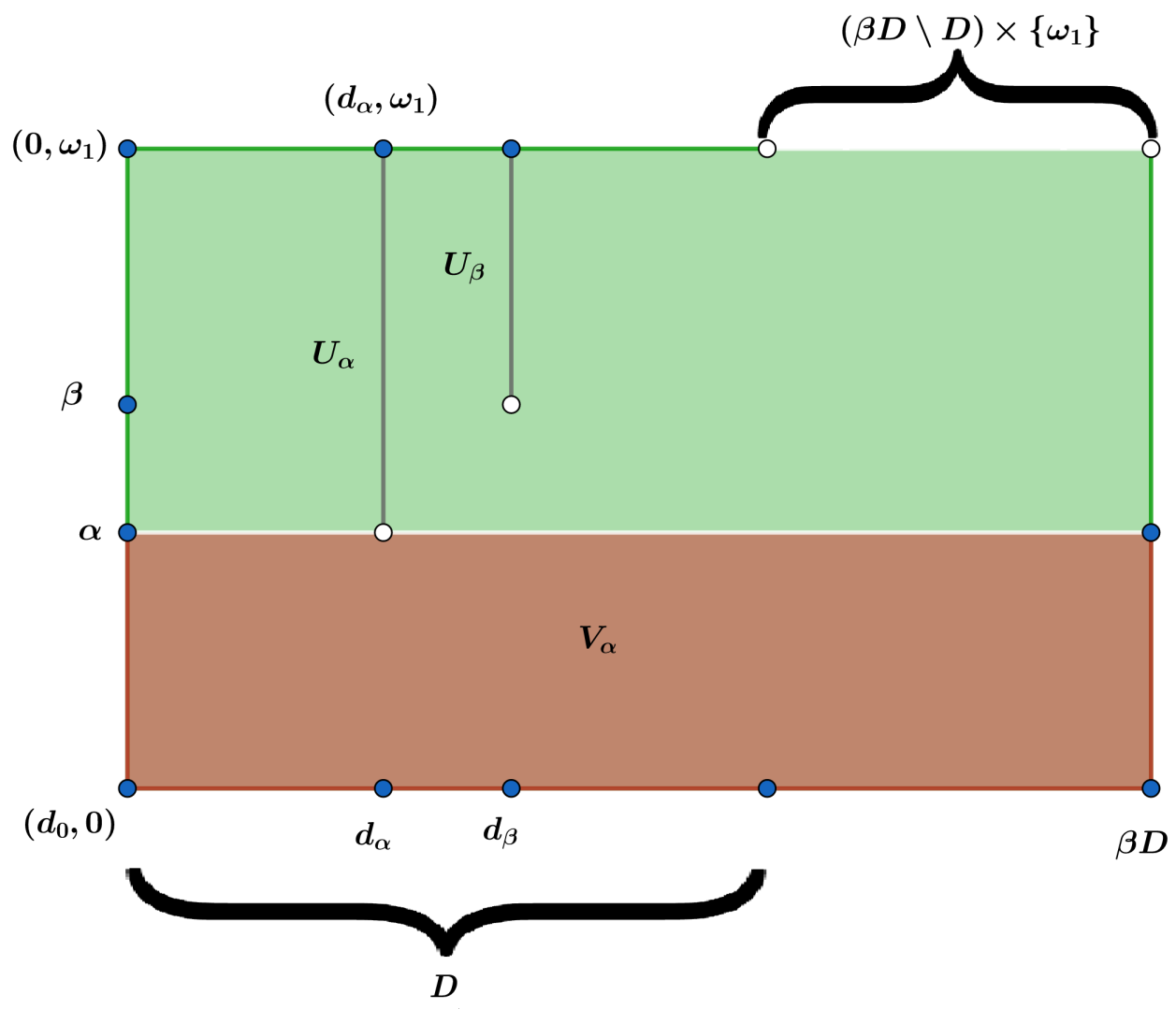

Figure 2.2: Espacio de Tychonoff pseudocompacto no 1-estrella numerable 
24 CAPÍTULO 2. PROPIEDADES ESTRELLA P Y PSEUDOCOMPACIDAD. 


\section{Capítulo 3}

\section{Espacios estrella $\mathbf{P}$ y la propiedad de Lindelöf.}

\subsection{Espacios estrella numerable y estrella Lindelöf}

En esta sección estudiaremos las clases de los espacios estrella numerable, estrella Lindelöf y la relación que tienen éstas con otras clases de espacios topológicos.

Definición 3.1.1. Decimos que un espacio topológico $X$ es de Lindelöf si cada cubierta abierta tiene una subcubierta numerable.

Proposición 3.1.2. Si $X$ es un espacio de Lindelöf, entonces es estrella numerable.

Demostración. Sea $\mathcal{U}$ una cubierta abierta de $X$. Entonces, existe $\left\{V_{n}: n \in \omega\right\} \subseteq \mathcal{U}$ tal que $\bigcup\left\{V_{n}: n \in \omega\right\}=X$. Para cada $n \in \omega$, escogemos $v_{n} \in V_{n}$, y sea $A=\left\{v_{n}: n \in\right.$ $\omega\}$. Entonces $S t(A, \mathcal{U}) \supseteq \bigcup\left\{V_{n}: n \in \omega\right\}=X$. Por lo tanto $X$ es estrella numerable.

Definición 3.1.3. Decimos que un espacio topológico $X$ es $\sigma$-compacto si es la unión numerable de subespacios compactos.

Es evidente que un espacio numerable es $\sigma$-compacto y de Lindelöf. Así que el siguiente lema es consecuencia inmediata de la aseveración anterior.

Lema 3.1.4. Si $X$ es un espacio estrella numerable, entonces $X$ es estrella $\sigma$-compacto y también es estrella Lindelöf.

El siguiente ejemplo muestra que la propiedad de ser estrella $\sigma$-compacto y la de ser estrella Lindelöf no son equivalentes.

Ejemplo 3.1.5. Existe un espacio de Tychonoff que es estrella Lindelöf, pero no es estrella $\sigma$-compacto. 
Demostración. Sean $Y=\left(\omega_{1}+1, \tau_{\omega}\right)$ donde $\tau_{\omega}$ es la topología generada por los subconjuntos $G_{\delta}$ en la topología del orden, $\omega_{2}+1$ con la topología del orden. Definimos $Z=\left(\left(\omega_{2}+1\right) \times Y\right) \backslash\left\{\left(\omega_{2}, \omega_{1}\right)\right\}$.

Notemos que cada subconjunto numerable de $Y$ es cerrado y discreto y en consecuencia, un subespacio compacto de $\omega_{1}+1$ tiene que ser finito. Para mostrar que $Z$ es estrella Lindelöf supongamos que $\mathcal{V}$ es una cubierta abierta de $Z$. Para cada $\alpha \in \omega_{1} \subset \omega_{1}+1$ podemos elegir $V_{\alpha} \in \mathcal{V}$ de modo que $\left(\omega_{2}, \alpha\right) \in V_{\alpha}$ y $\beta_{\alpha} \in \omega_{2}$ tal que $\left(\beta_{\alpha}, \omega_{2}\right] \times\{\alpha\} \subseteq V_{\alpha}$. Sean $\beta=\sup \left\{\beta_{\alpha}: \alpha \in \omega_{1}\right\} \in \omega_{2}$ y $A=\{\beta+1\} \times\left(\omega_{1}+1\right)$, entonces $S=\left(\beta+1, \omega_{2}\right] \times \omega_{1} \subseteq S t(A, \mathcal{V})$.

Además $Z \backslash S$ es la unión de los subespacios $[0, \beta+1] \times\left(\omega_{1}+1\right)$ y $\omega_{2} \times\left\{\omega_{1}\right\}$ y el primero es un espacio de Lindelöf, mientras que el segundo es numerablemente compacto. Así existe un conjunto $C \subset Z$ numerable y un conjunto finito $F \subset Z$ tales que $Z \backslash S \subseteq S t(C, \mathcal{V}) \cup S t(F, \mathcal{V})$ y así $A \cup C \cup F$ es el subespacio de Lindelöf en $Z$ que es un núcleo para la cubierta abierta $\mathcal{V}$.

Para terminar basta ver que $Z$ no es estrella $\sigma$-compacto y para ello es suficiente considerar la cubierta $\mathcal{U}=\left\{\omega_{2} \times\left(\omega_{1}+1\right)\right\} \cup\left\{\left(\omega_{2}+1\right) \times\{\alpha\}: \alpha \in \omega_{1}\right\}$. Supongamos que $R=\bigcup\left\{R_{n}: n \in \omega\right\}$ es un núcleo para la cubierta abierta $\mathcal{U}$, donde $R_{n}$ es un subespacio compacto para cada $n \in \omega$. Como $\pi_{Y}\left(R_{n}\right)$ es finito para cada $n \in \omega$, entonces el subconjunto $\pi_{Y}(R)$ está contenido en un subconjunto numerable de $Y$, por lo que existe $\alpha \in \omega_{1}$ tal que $\alpha>\beta$ para cada $\beta \in \pi_{Y}(R)$. Por lo tanto $\left(\omega_{2}, \alpha\right) \notin S t(R, \mathcal{U})$, lo que es una contradicción.

Lema 3.1.6. Sea $X$ un espacio topológico $T_{1}$ y sea $\mathcal{F}=\left\{F_{\alpha}: \alpha<\kappa\right\}$ una familia localmente finita. Si para cada $\alpha<\kappa$, elegimos $x_{\alpha} \in F_{\alpha}$, entonces el conjunto $D=$ $\left\{x_{\alpha}: \alpha<\kappa\right\}$ es un subconjunto cerrado y discreto.

Demostración. Primero demostremos que $D$ es un subconjunto cerrado. Sea $z \in$ $X \backslash D$. Entonces, existe $U \in \tau(z, X)$ y una subfamilia $\left\{F_{\alpha_{1}}, \ldots, F_{\alpha_{n}}\right\} \subseteq \mathcal{F}$ tal que si $\alpha \in \kappa \backslash\left\{\alpha_{1}, \ldots, \alpha_{n}\right\}$, entonces $F_{\alpha} \cap U=\emptyset$; así el conjunto $V=U \backslash\left\{x_{\alpha_{1}}, \ldots, x_{\alpha_{n}}\right\}$ es abierto, contiene a $z$ y es ajeno a $D$.

Para ver que $D$ es discreto, sea $x_{\beta} \in D$. Entonces existe $W \in \tau\left(x_{\beta}, X\right)$ y una subfamilia $\left\{F_{\beta_{1}}, \ldots, F_{\beta_{m}}\right\} \subseteq \mathcal{F}$ tal que si $\alpha \in \kappa \backslash\left\{\beta_{1}, \ldots, \beta_{m}\right\}$, entonces $F_{\alpha} \cap W=\emptyset$. Así el conjunto $O=W \backslash\left(\left\{x_{\beta_{j}}: 1 \leq j \leq n\right\} \backslash\left\{x_{\beta}\right\}\right)$ cumple $O \cap D=\left\{x_{\beta}\right\}$. Por lo tanto $D$ es discreto.

A continuación mostraremos una clase de espacios contenida en la clase de los espacios estrella Lindelöf y algunas consecuencias de la siguiente observación: cada partición abierta (cada elemento de la partición es un subconjunto abierto) de un espacio 
topológico es una familia discreta de subconjuntos abiertos y por lo tanto es una familia localmente finita.

Definición 3.1.7. Decimos que un espacio topológico $X$ es tenuemente Lindelöf si cada famila localmente finita de subconjuntos abiertos no vacíos es numerable.

Teorema 3.1.8. Si $X$ es un espacio $T_{1}$ estrella Lindelöf, entonces $X$ es tenuemente Lindelöf.

Demostración. Supongamos lo contrario, que existe una familia localmente finita de subconjuntos abiertos no vacíos $\mathcal{F}$ de tamaño $\omega_{1}$. Por el Lema 1.2.6, existe una familia celular localmente finita $\mathcal{F}^{\prime}=\left\{F_{\alpha}: \alpha<\omega_{1}\right\}$. Para cada $\alpha<\omega_{1}$, elegimos $x_{\alpha} \in F_{\alpha}$, entonces el conjunto $A=\left\{x_{\alpha}: \alpha<\omega_{1}\right\}$ es un conjunto cerrado y discreto por el Lema 3.1.6.

Sea $\mathcal{V}=\{X \backslash A\} \cup\left\{F_{\alpha}: \alpha \in \omega_{1}\right\}$. Si $N$ es un núcleo para $\mathcal{V}$, entonces para cada $\alpha<\omega_{1}$ se tiene $N \cap F_{\alpha} \neq \emptyset$. Para cada $\alpha<\omega_{1}$, sea $d_{\alpha} \in N \cap F_{\alpha}$, entonces $D=\left\{d_{\alpha}: \alpha<\omega_{1}\right\}$ es un subconjunto cerrado y discreto en $N$. Por lo tanto, $N$ no es de Lindelöf. Así, $X$ no es estrella Lindelöf.

Corolario 3.1.9. Si $X$ es un espacio estrella numerable, entonces $X$ es tenuemente Lindelöf.

Demostración. Se sigue del Lema 3.1.4 y del Teorema 3.1.8.

Corolario 3.1.10. Si $X$ es un espacio topológico que posee una partición abierta no numerable, entonces $X$ no es estrella Lindelöf.

Corolario 3.1.11. El producto de un espacio de Lindelöf y un espacio numerablemente compacto no necesariamente es estrella Lindelöf.

Demostración. Sean $Y=\left(\omega_{1}+1, \tau_{\omega}\right)$ como en el Ejemplo 3.1 .5 y $X=\omega_{1} \times Y$. Tomando la siguiente partición abierta $\mathcal{P}=\left\{U_{\alpha}: \alpha \in \omega_{1}\right\} \cup\{V\}$, donde $V=\{(\alpha, \beta)$ : $\alpha \in \omega_{1}, \beta \in \omega_{1}+1$ y $\left.\beta \geq \alpha\right\}$ y para cada $\alpha \in \omega_{1} U_{\alpha}=\left[\alpha+1, \omega_{1}\right) \times\{\alpha\} ; \mathcal{P}$ es no numerable y por lo tanto $X$ no es estrella Lindelöf.

Definición 3.1.12. Un espacio $X$ es $\omega_{1}$-Lindelöf si cada cubierta abierta de tamaño $\omega_{1}$ tiene una subcubierta numerable.

Teorema 3.1.13. Si $X$ es un espacio $\omega_{1}$-Lindelöf, entonces $e(X)=\omega$.

Demostración. Supongamos que $D=\left\{d_{\alpha}: \alpha<\omega_{1}\right\}$ es un subespacio cerrado y discreto de $X$, entonces para cada $\alpha<\omega_{1}$, existe $V_{\alpha} \in \tau\left(d_{\alpha}, X\right)$ tal que $V_{\alpha} \cap D=\left\{d_{\alpha}\right\}$. Como $X \backslash D$ es un subconjunto abierto tenemos que $\mathcal{V}=\left\{V_{\alpha}: \alpha<\omega_{1}\right\} \cup\{X \backslash D\}$ es una cubierta abierta de $X$ de tamaño $\omega_{1}$, que no posee una subcubierta numerable. 
Proposición 3.1.14. Si $P$ es una propiedad topológica que se preserva bajo imágenes continuas, entonces la propiedad estrella $P$ también se preserva bajo imágenes continuas.

Demostración. Sea $f: X \rightarrow Y$ una función continua y sobreyectiva, supongamos que $P$ es una propiedad topológica que se preserva bajo funciones continuas y que además $X$ es estrella $P$.

Sea $\mathcal{V}$ una cubierta abierta de $Y$. Entonces $\mathcal{U}=\left\{f^{-1}[V]: V \in \mathcal{V}\right\}$ es una cubierta abierta de $X$. Por ser $X$ estrella $P$, entonces existe un subconjunto $A \subseteq X$ con la propiedad $P$ tal que $X=S t(A, \mathcal{U})=\bigcup\left\{f^{-1}[V]: f^{-1}[V] \cap A \neq \emptyset\right\}$.

Observemos que $f[A]$ tiene $P$ y $S t(f[A], \mathcal{V})=\bigcup\{V \in \mathcal{V}: V \cap f[A] \neq \emptyset\}=Y$.

Con este resultado se ha probado que la imagen continua de un espacio estrella numerable (estrella compacto, estrella $\sigma$-compacto, estrella Lindelöf) es estrella numerable (respectivamente, estrella compacto, estrella $\sigma$-compacto, estrella Lindelöf).

Proposición 3.1.15. Un espacio $X$ es estrella numerable si y sólo si es estrella separable.

Demostración. $(\Rightarrow)$ Es inmediato.

$(\Leftarrow)$ Sea $\mathcal{U}$ una cubierta abierta de $X$, entonces existe $A \subseteq X$ separable tal que $S t(A, \mathcal{U})=X$. Sea $D$ el subconjunto denso numerable de $\bar{A}$. Si $x \in X$, entonces existe $a \in A$ tal que $x \in S t(a, \mathcal{U})$, así que existe $U \in \mathcal{U}$ tal que $a, x \in U$. Como $D$ es denso en $A$, existe $d \in D$ tal que $d \in U$, así $x \in S t(D, \mathcal{U})$.

Definición 3.1.16. Decimos que una propiedad topológica $P$ es compactamente productiva si para cada espacio topológico $X$ que tiene la propiedad $P$ y cada espacio compacto $K, X \times K$ tiene la propiedad $P$.

Observación 3.1.17. Supongamos que $K$ es un espacio compacto y $\mathcal{W}=\{U \times V: U \in$ $\mathcal{B}_{X}$ y $\left.V \in \mathcal{B}_{K}\right\}$ es una cubierta de $X \times K$, donde $\mathcal{B}_{X}$ y $\mathcal{B}_{K}$ son bases para las topologías de $X$ y $K$ respectivamente. Sean $x \in X$ y $\mathcal{W}_{x}=\{U \times V \in \mathcal{W}:(U \times V) \cap(\{x\} \times K) \neq \emptyset\}$, así la colección $\mathcal{V}=\left\{V: U \times V \in \mathcal{W}_{x}\right\}$ es una cubierta abierta de $K$ por lo que podemos encontrar una subcubierta finita $\left\{V_{1}(x), \ldots, V_{n_{x}}(x)\right\} \subseteq \mathcal{V}$. Ahora notemos que $\left\{U_{k}(x) \times V_{k}(x): 1 \leq k \leq n_{x}\right\} \subseteq \mathcal{W}_{x}$ es una subcubierta finita de $\{x\} \times K$. Mas aún, si tomamos $O_{x}=\bigcap\left\{U_{k}(x): 1 \leq k \leq n_{x}\right\}$ tenemos que $O_{x} \times K \subseteq \bigcup\left\{U_{k}(x) \times V_{k}(x): 1 \leq\right.$ $\left.k \leq n_{x}\right\}$.

Teorema 3.1.18. Si $K$ es compacto y $X$ es estrella $P$, donde $P$ es una propiedad topológica compactamente productiva, entonces $X \times K$ también es estrella $P$. 
Demostración. Sea $X$ un espacio estrella $P$ y $K$ un espacio compacto. Sea $\mathcal{W}$ una cubierta abierta de $X \times K$ por abiertos básicos. Usando la notación de la Observación 3.1.17, sea $\mathcal{O}=\left\{O_{x}: x \in X\right\}$. Ya que $X$ es estrella $P$, existe un subespacio $A \subseteq X$ con la propiedad $P$ tal que $S t(A, \mathcal{O})=X$. Entonces $A \times K$ tiene $P$ y $S t(A \times K, \mathcal{W})=X \times K$.

Corolario 3.1.19. El producto de un espacio estrella Lindelöf (estrella $\sigma$-compacto, estrella numerablemente compacto) y un espacio compacto es estrella Lindelöf (respectivamente estrella $\sigma$-compacto, estrella numerablemente compacto).

Teorema 3.1.20. El producto de un espacio estrella numerable $X$ y un espacio compacto y separable $K$ es estrella numerable.

Demostración. Sea $\mathcal{U}$ una cubierta abierta de $X \times K$ por abiertos básicos y usando de nuevo la notación de la Observación 3.1.17, sea $\mathcal{O}=\left\{O_{x}: x \in X\right\}$. Entonces existe $A \subseteq X$ numerable tal que $\operatorname{St}(A, \mathcal{O})=X$ y existe un subespacio denso numerable $D \subseteq K$. Así $A \times D$ es numerable y tenemos que $\operatorname{St}(A \times D, \mathcal{U})=X$.

Corolario 3.1.21. El producto de un espacio estrella separable $X$ y un espacio compacto y separable $K$ es estrella separable.

Teorema 3.1.22. Si $X$ es un espacio tenuemente Lindelöf y $Y$ es separable, entonces $X \times Y$ es tenuemente Lindelöf.

Demostración. Supongamos lo contrario, es decir, que existe una familia localmente finita de subconjuntos abiertos no vacíos $\mathcal{C}$ no numerable en $X \times Y$. Podemos asumir, sin pérdida de generalidad, que $|\mathcal{C}|=\omega_{1}$ y que cada elemento de $\mathcal{C}$ es un abierto básico en el producto, esto es:

$$
\mathcal{C}=\left\{U_{\alpha} \times V_{\alpha}: \alpha \in \omega_{1}\right\}
$$

donde $U_{\alpha}$ es un conjunto abierto en $X$ y $V_{\alpha}$ es un conjunto abierto en $Y$. Sea $D \subseteq Y$ un conjunto denso numerable. Para cada $d \in D$, consideremos a la familia $\left\{U_{\alpha}: \alpha \in \omega_{1} \mathrm{y}\right.$ $\left.d \in V_{\alpha}\right\}$. Supongamos que la familia $\left\{U_{\alpha}: \alpha \in \omega_{1}\right.$ y $\left.d \in V_{\alpha}\right\}$ no es localmente finita en $X$, entonces existe $x \in X$ tal que para cada $W \in \tau(x, X), W$ intersecta una cantidad infinita de elementos de $\left\{U_{\alpha}: \alpha \in \omega_{1} \mathrm{y} d \in V_{\alpha}\right\}$; entonces cualquier vecindad de $(x, d)$ intersecta una cantidad infinita de elementos de $\mathcal{C}$, lo que contradice que $\mathcal{C}$ es una familia localmente finita. Como el espacio $X$ es tenuemente Lindelöf, entonces la familia $\left\{U_{\alpha}: \alpha \in \omega_{1}\right.$ y $\left.d \in V_{\alpha}\right\}$ es numerable.

Veamos que el conjunto $I=\left\{\alpha \in \omega_{1}: d \in V_{\alpha}\right\}$ es finito para cada $d \in D$. Para ello supongamos lo contrario, es decir, que existe $d_{0} \in D$ tal que $\left\{\alpha \in \omega_{1}: d_{0} \in V_{\alpha}\right\}$ es infinito. Como la familia $\left\{U_{\alpha}: \alpha \in \omega_{1}\right.$ y $\left.d \in V_{\alpha}\right\}$ es numerable, entonces existe $J \subseteq I$ tal que la cardinalidad de $J$ es infinita y un subconjunto abierto $O$ tal que 
si $\alpha \in J$ se tiene que $U_{\alpha}=O$. Sea $x \in O$, entonces para todo $\alpha \in J$ se tiene que $\left(x, d_{0}\right) \in U_{\alpha} \times V_{\alpha}$. Esto es una contradicción ya que la familia $\mathcal{C}$ es localmente finita. Por lo tanto el conjunto $\left\{\alpha \in \omega_{1}: d \in V_{\alpha}\right\}$ es finito para cada $d \in D$. Se sigue que $\omega_{1}=\bigcup\left\{\left\{\alpha \in \omega_{1}: d \in V_{\alpha}\right\}: d \in D\right\}$, el cual es numerable, lo que nos lleva a una contradicción.

Al principio de esta sección se mostró que cada espacio estrella numerable es estrella $\sigma$-compacto y estrella Lindelöf, después exhibimos un ejemplo de un espacio estrella Lindelöf que no es estrella $\sigma$-compacto y naturalmente nace la pregunta ¿todo espacio estrella $\sigma$-compacto es estrella numerable?, la respuesta es que estas dos propiedades topológicas no son equivalentes y a continuación se muestra un ejemplo de esto.

Ejemplo 3.1.23. Existe un espacio de Tychonoff estrella $\sigma$-compacto que no es estrella numerable.

Demostración. Sean $\Psi(\mathcal{A})=\omega \cup \mathcal{A}$ un espacio de Mrowka, donde $\mathcal{A}$ es una familia MAD en $\omega$ de cardinalidad $\mathfrak{c}, Y$ la compactación unipuntual del espacio discreto $D$ de cardinalidad $\mathfrak{c}$ y $X=\Psi(\mathcal{A}) \times Y$. Como $\Psi(\mathcal{A})$ es separable, entonces es estrella numerable y por lo tanto es estrella $\sigma$-compacto. Como la propiedad de ser estrella $\sigma$ compacto es compactamente productiva, concluimos que $X$ es estrella $\sigma$-compacto. Si $\left\{A_{\alpha}: \alpha \in \mathfrak{c}\right\}$ y $\left\{d_{\alpha}: \alpha \in \mathfrak{c}\right\}$ son enumeraciones de $\mathcal{A}$ y de $D$ respectivamente, entonces la cubierta

$\mathcal{U}=\left\{\Psi(\mathcal{A}) \times\left\{d_{\alpha}\right\}: \alpha<\mathfrak{c}\right\} \cup\left\{\left(A_{\alpha} \cup\left\{A_{\alpha}\right\}\right) \times\left(Y \backslash\left\{d_{\alpha}\right\}\right): \alpha<\mathfrak{c}\right\} \cup\{\{n\} \times Y: n \in \omega\}$

no posee núcleo numerable, pues si $C$ es un subconjunto numerable en $X$, tenemos que $B=\left\{\alpha: d_{\alpha} \notin \pi_{2}[C]\right\}$ es no numerable. Sea $\beta \in B$, entonces $\left(A_{\beta}, d_{\beta}\right) \notin S t(C, \mathcal{U})$.

Teorema 3.1.24. Cada espacio de Tychonoff puede estar inmerso como un subconjunto cerrado y $G_{\delta}$ en un espacio de Tychonoff estrella $\sigma$-compacto.

Demostración. Sea $Y=(\beta X \times \omega) \cup(X \times\{\omega\})$, donde $Y$ tiene la topología relativa como subespacio de $\beta X \times(\omega+1)$. Observemos que $\beta X \times \omega$ es un subconjunto $\sigma$ compacto y denso en $Y$, por lo tanto es un núcleo $\sigma$-compacto para cada cubierta abierta de $Y$.

Definición 3.1.25. Decimos que $X$ es un $P$-espacio si cada subconjunto $G_{\delta}$ es abierto.

Observemos que en un $P$-espacio la unión de $\omega$ subconjuntos cerrados es un subconjunto cerrado como consecuencia de las leyes de De Morgan.

Definición 3.1.26. Un espacio topológico $X$ se llama 0-dimensional si es $T_{1}$ y tiene una base que consiste de conjuntos abiertos-cerrados. 
Lema 3.1.27. $\mathrm{Si} \quad X$ es un $P$-espacio de Tychonoff, entonces $X$ es un espacio 0-dimensional.

Demostración. Sean $x \in X$ y $U \in \tau(x, X)$. Como $X$ es un espacio completamente regular, existe una función continua $f: X \rightarrow[0,1]$ tal que $f(x)=0$ y $f(y)=1$ para cada $y \in X \backslash U$. Entonces $f^{-1}\left[\left[0, \frac{1}{2}\right]\right.$ es una vecindad cerrada de $x$ contenida en $U$, pero como $\left[0, \frac{1}{2}\right]=\bigcap\left\{\left[0, \frac{1}{2}+\frac{1}{n}\right): n \in \omega\right\}$, entonces

$$
f^{-1}\left[\left[0, \frac{1}{2}\right]\right]=f^{-1}\left[\bigcap\left\{\left[0, \frac{1}{2}+\frac{1}{n}\right): n \in \omega\right\}\right]=\bigcap\left\{f^{-1}\left[\left[0, \frac{1}{2}+\frac{1}{n}\right)\right]: n \in \omega\right\} .
$$

Así $f^{-1}\left[\left[0, \frac{1}{2}\right]\right.$ es la intersección numerable de subconjuntos abiertos, ya que $X$ es un $P$-espacio se tiene que $f^{-1}\left[\left[0, \frac{1}{2}\right]\right.$ es abierto, por lo tanto $X$ es 0 -dimensional.

Hasta este momento hemos demostrado las siguientes implicaciones en la clase de los espacios $T_{1}$ :

$$
\begin{gathered}
\text { Extensión numerable } \Rightarrow \text { Estrella numerable } \Rightarrow \text { Estrella } \sigma \text {-compacto } \\
\Rightarrow \text { Estrella Lindelöf } \Rightarrow \text { Tenuemente Lindelöf. }
\end{gathered}
$$

El siguiente teorema nos porporciona condiciones necesarias y suficientes para obtener las implicaciones recíprocas en el diagrama anterior.

Teorema 3.1.28. Un $P$-espacio normal $X$ es tenuemente Lindelöf si y sólo si $e(X)=\omega$.

Demostración. $(\Rightarrow)$ Supongamos que $D=\left\{d_{\alpha}: \alpha<\omega_{1}\right\}$ es un subespacio cerrado y discreto de $X$. Por el Lema 3.1.27, $X$ es un espacio 0-dimensional. Elegimos un conjunto cerrado-abierto $U_{0}$ tal que $U_{0} \cap D=\left\{d_{0}\right\}$. Supongamos que para cada número ordinal $\alpha<\beta<\omega_{1}$ hemos elegido subconjuntos cerrado-abiertos ajenos $U_{\alpha}$ tales que $U_{\alpha} \cap D=\left\{d_{\alpha}\right\}$, como $X$ es un $P$-espacio se sigue que $\bigcup\left\{U_{\alpha}: \alpha<\beta\right\}$ es un subconjunto cerrado; por lo cual podemos encontrar un subconjunto abierto $U_{\beta}$ tal que $U_{\beta} \cap D=\left\{d_{\beta}\right\}$ y $U_{\beta} \cap U_{\alpha}=\emptyset$ para cada $\alpha<\beta$. Así $\mathcal{U}=\left\{U_{\alpha}: \alpha<\omega_{1}\right\}$ es una familia mutuamente ajena de subconjuntos cerrado-abiertos. Los subconjuntos cerrados $X \backslash \bigcup \mathcal{U}$ y $D$ son ajenos por lo que existen subconjuntos abiertos ajenos $U$ y $V$ tales que $D \subseteq U$ y $X \backslash \bigcup \mathcal{U} \subset V$, así $\mathcal{V}=\left\{U_{\alpha} \cap U: \alpha<\omega_{1}\right\}$. Sea $x \in X$, si $x \in \backslash \bigcup \mathcal{U}$, entonces $x \in V$ y $V \cap\left(U_{\alpha} \cap U\right)=\emptyset$ para cada $\alpha<\omega_{1}$; si $x \in \bigcup \mathcal{U}$, entonces existe $\beta<\omega_{1}$ tal que $x \in U_{\beta}$ y esta es una vecindad de $x$ que solamente intersecta a $U_{\beta} \cap U$, por lo tanto $\mathcal{V}$ es una familia localmente finita.

$(\Leftarrow)$ Sea $\mathcal{U}=\left\{U_{\alpha}: \alpha<\omega_{1}\right\}$ una familia localmente finita de subconjuntos abiertos no vacíos en $X$, si para cada $\alpha<\omega_{1}$ elegimos $d_{\alpha} \in U_{\alpha}$, por el Lema 3.1.6 tenemos que el subconjunto $D=\left\{d_{\alpha}: \alpha<\omega_{1}\right\}$ es cerrado y discreto en $X$. 
Notemos que para demostrar la suficiencia en el enunciado del Teorema 3.1.28 no es necesario que el espacio $X$ sea un $P$-espacio. Una pregunta interesante que aparece en [3] es la siguiente:

(i) ¿Es cierto que todo $P$-espacio tenuemente Lindelöf es estrella Lindelöf? Para poder responder a esta pregunta es necesaria la siguiente definición.

Definición 3.1.29. Sea $\kappa$ un cardinal infinito; una familia $\mathcal{A} \subset[\kappa]^{\kappa}$ es una familia casi ajena generalizada (CAG) si para cada $A, B \in \mathcal{A}$ se tiene que $|A \cap B|<\kappa$.

Una familia $C A G \mathcal{A}$ es maximal casi ajena generalizada $(M C A G)$ si $\mathcal{A} \subseteq \mathcal{B}$ y $\mathcal{B}$ es $C A G$ implica que $\mathcal{A}=\mathcal{B}$.

Notemos que esta definición es muy parecida a la de una familia casi ajena definida en el Capítulo 1, es por eso que para diferenciarlas usamos las siglas en español. Como consecuencia del Lema De Zorn se puede demostrar de forma análoga a las familias casi ajenas que cada familia casi ajena generalizada está contenida en una familia maximal casi ajena generalizada. Si $\mathcal{A} \subseteq\left[\omega_{1}\right]^{\omega_{1}}$ es una familia $M C A G$ en $\omega_{1}$ de cardinalidad $\kappa$, tal que $\bigcup \mathcal{A}=\omega_{1}$, entonces podemos definir una topología $\tau$ en $X=\omega_{1} \cup \mathcal{A}$ de la siguiente manera:

(1) Si $\alpha \in \omega_{1}$, entonces $\{\alpha\}$ es abierto.

(2) Si $A \in \mathcal{A}$, entonces una vecindad básica de $A$ es de la forma $\{A\} \cup(A \backslash C)$, donde $C$ es un subconjunto numerable.

El espacio $X$ se llama un espacio tipo Mröwka en $\omega_{1}$.

La respuesta a la pregunta $(i)$ es negativa bajo una suposición importante presentada en el enunciado del siguiente ejemplo.

Ejemplo 3.1.30. Si existe una familia $M C A G \mathcal{A}$ en $\omega_{1}$ tal que $|\mathcal{A}|^{\omega}=|\mathcal{A}|=\kappa$, entonces existe un $P$-espacio de Tychonoff tenuemente Lindelöf que no es estrella Lindelöf.

Demostración. Sea $X=\omega_{1} \cup \mathcal{A}$ un espacio tipo Mröwka en $\omega_{1}$. Primero observemos que $X$ es tenuemente Lindelöf, pues si $\mathcal{W}$ es una familia de tamaño $\omega_{1}$ de puntos aislados, entonces existe $A \in \mathcal{A}$ tal que $|A \cap(\bigcup \mathcal{W})|=\omega_{1}$. Dado que $A$ tiene punto de acumulación $p$, se sigue que $p$ también es punto de acumulación de la familia $\mathcal{W}$. Para ver que $X$ es un $P$-espacio basta notar que para cada $A \in \mathcal{A}$ la intersección numerable de vecindades de $A \in \mathcal{A}$ es una vecindad de $A$.

Para ver que el espacio $X$ no es estrella Lindelöf, como $\bigcup \mathcal{A}=\omega_{1}$ se tiene que $\{A \cup\{A\}: A \in \mathcal{A}\}$ es una cubierta abierta de $X$. Por lo tanto cada subespacio de Lindelöf de $X$ tiene una cubierta abierta numerable de la forma $\{A \cup\{A\}: A \in \mathcal{F}\}$, para alguna subfamilia numerable $\mathcal{F} \subset \mathcal{A}$. Además cada subconjunto $\bigcup\{F \cup\{F\}: F \in \mathcal{F}\}$ 
es de Lindelöf y cerrado en $X$ por ser la unión numerable de subconjuntos cerrados en un $P$-espacio. Se sigue que si $L \subseteq X$ es un subespacio de Lindelöf, entonces existe una subcolección numerable $\mathcal{F} \subseteq \mathcal{A}$ tal que $L \subseteq \bigcup\{F \cup\{F\}: F \in \mathcal{F}\}$. Como $\kappa^{\omega}=\kappa$ se sigue que a lo más hay $\kappa$ subespacios de este tipo en $X$.

Ahora vamos a definir $\phi: \mathcal{A} \rightarrow[\mathcal{A}]^{\omega}$ de tal forma que para cada $A \in \mathcal{A}, A \notin \phi(A)$. Para hacer esto, enumeramos los elementos de $\mathcal{A}$ y los elementos de $[\mathcal{A}]^{\omega}$ de la siguiente forma $\mathcal{A}=\left\{A_{\alpha}: \alpha \in \kappa\right\}$ y $[\mathcal{A}]^{\omega}=\left\{\mathcal{G}_{\beta}: \beta \in \kappa\right\}$. Para cada $A_{\alpha}$ definimos $\phi\left(A_{\alpha}\right)=\mathcal{G}_{\beta_{\alpha}}$, donde $\beta_{\alpha} \in \kappa$ es el mínimo ordinal tal que $A_{\alpha} \notin \mathcal{G}_{\beta_{\alpha}}$ y $\mathcal{G}_{\beta_{\alpha}} \neq \mathcal{G}_{\beta_{\gamma}}$ para cada $\gamma<\alpha$. Notemos que podemos hacer esta elección ya que para cada $\alpha<\kappa$, se tiene que $\left|\left\{\xi \in \kappa: A_{\alpha} \notin \mathcal{G}_{\xi}\right\}\right|=\kappa$. Supongamos que $\phi$ no es sobreyectiva, entonces existe $\mathcal{G}_{\beta} \in[\mathcal{A}]^{\omega}(\beta<\kappa)$ tal que para cada $\alpha<\kappa, \phi\left(A_{\alpha}\right) \neq \mathcal{G}_{\beta}$, es decir, $\beta \neq \beta_{\alpha}$ para cada $\alpha<\kappa$. Sea $\mathcal{G}_{\beta}=\left\{A_{\beta_{n}}: n \in \omega\right\}$. Para cada $\alpha \in \kappa \backslash\left\{\beta_{n}: n \in \omega\right\}$ tenemos que $A_{\alpha} \notin \mathcal{G}_{\beta}$; por lo que $\beta_{\alpha}<\beta<\kappa$ para cada $\alpha<\kappa$. Esto es imposible, pues $|\beta|<\kappa \mathrm{y}$ $\left|\kappa \backslash\left\{\beta_{n}: n \in \omega\right\}\right|=\kappa$. Se sigue que $\phi$ es sobreyectiva.

Para cada $\alpha \in \kappa$ definimos:

$$
U_{\alpha}=\left\{A_{\alpha}\right\} \cup\left(A_{\alpha} \backslash \bigcup\left\{B \cap A_{\alpha}: B \in \phi\left(A_{\alpha}\right)\right\}\right) .
$$

Como $\mathcal{A}$ es una familia MCAG, cada $U_{\alpha}$ es un subconjunto abierto. Ahora definimos:

$$
\mathcal{U}=\left\{U_{\alpha}: \alpha \in \kappa\right\} \cup\left\{\{d\}: d \in \omega_{1}\right\} .
$$

Afirmamos que $\mathcal{U}$ es una cubierta abierta de $X$ que no posee un núcleo de Lindelöf. Para demostrar esta afirmación, supongamos lo contrario, es decir, que existe un subconjunto de Lindelöf $L$ tal que $S t(L, \mathcal{U})=X$. Entonces existe $\mathcal{F} \in[\mathcal{A}]^{\omega}$ tal que $L \subseteq \bigcup\{A \cup\{A\}$ : $A \in \mathcal{F}\}$ y existe $A_{\xi} \in \mathcal{A}$ tal que $\phi\left(A_{\xi}\right)=\mathcal{F}$. Además, $A_{\xi} \in S t(L, \mathcal{U})$ por lo que existe $d \in L$ y $U \in \mathcal{U}$ tal que $d, A_{\xi} \in U$. Necesariamente $U=U_{\xi}=\left\{A_{\xi}\right\} \cup\left(A_{\xi} \backslash \cup\left\{B \cap A_{\xi}\right.\right.$ : $\left.B \in \phi\left(A_{\xi}\right)\right\}$ ); entonces $d \in A_{\xi} \backslash \bigcup\left\{B \cap A_{\xi}: B \in \phi\left(A_{\xi}\right)\right\}$ y $d \in \bigcup \phi\left(A_{\xi}\right)$, lo que es una contradicción.

La existencia de una familia $M C A G \mathcal{A}$ en $\omega_{1}$ tal que $\left|\mathcal{A}^{\omega}\right|=|\mathcal{A}|$, es independiente de ZFC y corresponde al ejercicio B5 del capítulo 8 en la página 290 de [14].

Definición 3.1.31. Sean $(X, \tau)$ un espacio topológico, $F \subseteq X$ y $\mathcal{U} \subseteq \tau(F, X)$, decimos que $\mathcal{U}$ es una base de vencindades para $F$ si para cada subconjunto $V \in \tau(F, X)$ existe $U \in \mathcal{U}$ tal que $F \subseteq U \subseteq V$. Denotaremos

$$
\chi(F, X)=\min \{|\mathcal{U}|: \mathcal{U} \text { es una base de vecindades para } F\} .
$$

Si $x \in X$ escribiremos $\chi(x, X)$ en lugar de $\chi(\{x\}, X)$.

Se define el carácter del espacio topológico $X$ como:

$$
\chi(X)=\sup \{\chi(x, X): x \in X\} .
$$


Teorema 3.1.32. $\left(2^{\omega}<2^{\omega_{1}}\right)$ Si $X$ es un espacio normal, tenuemente Lindelöf y $\chi(X) \leq$ $\mathfrak{c}$, entonces $X$ tiene extensión numerable.

Demostración. Supongamos lo contrario, es decir, que $X$ es un espacio normal, tenuemente Lindelöf, $\chi(X) \leq \mathfrak{c}$ y $e(X) \geq \omega_{1}$. Sea $F$ un subconjunto cerrado y discreto de cardinalidad $\omega_{1}$. Para cada $x \in F$, sea $\mathcal{B}_{x}$ una base local en $x$ de cardinalidad a lo más c. Como $X$ es normal, para cada subconjunto no vacío $G \subset F$ fijamos un subconjunto abierto $U_{G}$ tal que $G \subseteq U_{G}$ y $\operatorname{cl}\left(U_{G}\right) \cap(F \backslash G)=\emptyset$. Para cada $G$ elegimos una colección maximal ajena dos a dos de elementos de $\bigcup\left\{\mathcal{B}_{x}: x \in G\right\}$ que esté contenida en $U_{G}$, denotamos a esta familia por $\mathcal{A}_{G}$. Veamos que $G \subseteq \operatorname{cl}\left(\bigcup \mathcal{A}_{G}\right)$. Para esto supongamos lo contrario, es decir, que existe $x \in G \backslash \operatorname{cl}\left(\bigcup \mathcal{A}_{G}\right) \subseteq U_{G} \backslash \operatorname{cl}\left(\bigcup \mathcal{A}_{G}\right)$, entonces existe $B \in \mathcal{B}_{x}$ tal que $B \subseteq U_{G} \backslash \operatorname{cl}\left(\bigcup \mathcal{A}_{G}\right)$, lo que contradice que $\mathcal{A}_{G}$ es una familia maximal. Entonces $G \subseteq \operatorname{cl}\left(\bigcup \mathcal{A}_{G}\right)$.

Sean $G, H \subset F$ y $G \nsubseteq H$ y tomemos $y \in G \backslash H$. Entonces $y \in U_{G}$ pero $y \notin U_{H}$ puesto que $\operatorname{cl}\left(U_{H}\right) \cap(F \backslash H)=\emptyset$. Como $y \in G \backslash H$, existe una vecindad $W$ de $y$ ajena a $H$, por lo que $y \notin \operatorname{cl}\left(\bigcup \mathcal{A}_{H}\right)$. Por lo tanto, $\mathcal{A}_{H}$ no es maximal para $G$. Se sigue que el mapeo $G \mapsto \mathcal{A}_{G}$ es inyectivo.

Ahora consideramos dos casos:

(1) Cada familia $\mathcal{A}_{G}$ es numerable; o

(2) Existe alguna familia $\mathcal{A}_{G}$ no numerable.

Supongamos que se cumple (1). Como $|F|=\omega_{1}$ y para cada $x \in F,\left|\mathcal{B}_{x}\right| \leq \mathfrak{c}$, se sigue que $\left|\bigcup\left\{\mathcal{B}_{x}: x \in F\right\}\right| \leq \mathfrak{c}$. Como $\mathcal{A}_{G}$ es una subcolección numerable de la colección $\bigcup\left\{\mathcal{B}_{x}: x \in G\right\}$ tenemos que $\left|\left\{\mathcal{A}_{G}: \emptyset \subsetneq G \subsetneq F\right\}\right| \leq \mathfrak{c}^{\omega}=\mathfrak{c}$. Además la función $G \mapsto \mathcal{A}_{G}$ es inyectiva, así que $\left|\left\{\mathcal{A}_{G}: \emptyset \subsetneq G \subsetneq F\right\}\right|=2^{\omega_{1}}$, lo que es una contradicción.

Si se cumple (2), entonces podemos elegir $\emptyset \subsetneq G \subsetneq F$, tal que $\mathcal{A}_{G}$ es no numerable, por lo tanto encontramos un subconjunto no numerable $A \subseteq G$ que esté separado por los elementos de $\mathcal{A}_{G}$. Como $X$ es normal, existen subconjuntos abiertos ajenos $U, V$ tales que $A \subseteq U$ y $X \backslash\left(\bigcup \mathcal{A}_{G}\right) \subseteq V$. Así la familia $\mathcal{U}=\left\{U \cap W: W \in \mathcal{A}_{G}\right\}$ es una familia localmente finita no numerable de subconjuntos abiertos.

Corolario 3.1.33. (CH) Si $X$ es un espacio normal, primero numerable y tenuemente compacto, entonces $X$ tiene extensión numerable.

En general hemos visto que un espacio que es tenuemente Lindelöf no necesariamente tiene extensión numerable. A continuación estudiamos una clase especial de subconjuntos de $\omega_{1} \times \omega_{1}$, pues en este espacio las últimas propiedades son equivalentes, para ello necesitamos los siguientes conceptos y un teorema que puede consultar en [12]. 
Definición 3.1.34. Un conjunto club de un cardinal regular no numerable $\kappa$ es un subconjunto $C \subseteq \kappa$ cerrado y no acotado.

Definición 3.1.35. Un conjunto $S \subset \kappa$ es estacionario si $S \cap C \neq \emptyset$ para cada subconjunto club $C$ de $\kappa$. Denotaremos por $S(\kappa)$ a la colección de todos los subconjuntos estacionarios de $\kappa$.

Teorema 3.1.36. [12]. Si $A, B \in S\left(\omega_{1}\right)$, entonces los siguientes enunciados son equivalentes:

1) $A \cap B \in S\left(\omega_{1}\right)$;

2) $A \times B$ es normal;

3) cada subconjunto no numerable de $A \times B$ tiene un punto de acumulación.

Teorema 3.1.37. Si $A, B \in S\left(\omega_{1}\right)$, entonces los siguientes enunciados son equivalentes:

1) $A \times B$ es tenuemente Lindelöf;

2) $A \times B$ tiene extensión numerable;

3) $A \times B$ es normal.

Demostración. Del Teorema 3.1 .28 se sigue $(2) \Rightarrow(1)$ y del Teorema anterior obtemos la equivalencia $(2) \Leftrightarrow(3)$. Así que basta probar $(1) \Rightarrow(3)$. Supongamos que $A, B \in S\left(\omega_{1}\right)$ pero $A \cap B \notin S\left(\omega_{1}\right)$. Sea $C$ un subconjunto club en $\omega_{1}$ tal que $C \cap A \cap B=\emptyset$ y enumeramos a $C$ como $\left\{\gamma_{\alpha}: \alpha \in \omega\right\}$. Ahora definimos:

$\alpha_{0}=\min (C \cap A)$,

$\beta_{0}=\min \left((C \cap B) \backslash\left(\alpha_{0}+1\right)\right)$

$\alpha_{\delta}=\min \left((C \cap A) \backslash\left(\sup \left\{\beta_{\mu}: \mu<\delta\right\}+1\right)\right)$,

$\beta_{\delta}=\min \left((C \cap B) \backslash\left(\sup \left\{\alpha_{\mu}: \mu \leq \delta\right\}+1\right)\right)$.

Veamos que el conjunto $D=\left\{\left(\alpha_{\delta}, \beta_{\delta}\right): \delta<\omega_{1}\right\}$ es cerrado y discreto. Por la construcción, tenemos que si $\left\{\left(\alpha_{\delta_{n}}, \beta_{\delta_{n}}\right)\right\} \rightarrow(\alpha, \beta)$, entonces $\alpha=\beta$, lo que implica que $\alpha \in A \cap B \cap C$ y esto claramente es una contradicción. Ahora consideremos los puntos de la forma $\left(\alpha_{\delta+1}, \beta_{\delta+1}\right) \in D$. Si $\left(\alpha_{\delta+1}, \beta_{\delta+1}\right)$ es un punto aislado en $A \times B$, definimos $\left(a_{\delta+1}, b_{\delta+1}\right)=\left(\alpha_{\delta+1}, \beta_{\delta+1}\right)$, de otra forma, como $A \times B$ es disperso, podemos 
elegir un punto aislado:

$$
\left(a_{\delta+1}, b_{\delta+1}\right) \in V_{\delta+1}=\left(\left(\alpha_{\delta}, \alpha_{\delta+1}\right] \times\left(\beta_{\delta}, \beta_{\delta+1}\right]\right) \cap(A \times B)
$$

Notemos que $\alpha_{\delta}<a_{\delta+1} \leq \alpha_{\delta+1}$ y $\beta_{\delta}<b_{\delta+1} \leq \beta_{\delta+1}$; estas desigualdades implican que si $\left\{\left(a_{\delta}, b_{\delta}\right): \delta<\omega_{1}\right\}$ tuviera un punto de acumulación, entonces $D=\left\{\left(\alpha_{\delta}, \beta_{\delta}\right): \delta<\omega_{1}\right\}$ también tendría un punto de acumulación pero $D$ es un subconjunto cerrado y discreto. Así la familia discreta no numerable de subconjuntos abiertos $\left\{\left(a_{\delta}, b_{\delta}\right): \delta<\omega_{1}\right\}$ prueba que $A \times B$ no es tenuemente Lindelöf.

Definición 3.1.38. Un espacio topológico $X$ es Hausdorff por colecciones si cada para cada subconjunto cerrado y discreto $C \subseteq X$ existe una familia celular que separa a los elementos del subconjunto $C$.

La prueba del siguiente teorema la omitiremos, ya que está fuera del propósito de este trabajo. Se puede consultar una demostración en [13].

Teorema 3.1.39. Todos los subespacios de $\omega_{1}^{2}$ son Hausdorff por colecciones.

Teorema 3.1.40. Un subespacio de $\omega_{1}^{2}$ es estrella Lindelöf si y sólo si tiene extensión numerable.

Demostración. Asumamos lo contrario, es decir, que $X \subseteq \omega_{1}^{2}$ es estrella Lindelöf y $e(X)>\omega$; sea $F \subset X$ un subconjunto cerrado y discreto de cardinalidad $\omega_{1}$. Por el Teorema 3.1.39 el subespacio $X$ es Hausdorff por colecciones, existe una familia de subconjuntos abiertos ajena dos a dos $\mathcal{F}=\left\{U_{x}: x \in F\right\}$ tal que $x \in U_{x}$ para cada $x \in F$. Consideremos la cubierta abierta $\mathcal{U}=\mathcal{F} \cup\{X \backslash F\}$; como $X$ es estrella Lindelöf, existe un subespacio de Lindelöf $L \subseteq X$ tal que $S t(L, \mathcal{U})=X$. Para cada $x \in F$, existe $a \in L$ y $U \in \mathcal{U}$ tal que $a, x \in U$. Sin embargo, esto implica que $U=U_{x}$ y por lo tanto $a \in U_{x}$. Se sigue que $\left\{U_{x} \cap L: x \in F\right\}$ es una familia celular no numerable en $L$. Esto es una contradicción, pues cada subespacio de Lindelöf de $\omega_{1}^{2}$ es numerable.

\subsection{Espacios estrella extensión numerable.}

En esta sección estudiaremos espacios estrella extensión numerable, que es una clase importante de espacios topológicos ya que yace entre la clase de los espacios estrella Lindelöf y la de los espacios tenuemente Lindelöf. Un espacio $X$ es estrella extensión numerable si para cada cubierta abierta $\mathcal{U}$ de $X$ existe un subconjunto $A \subseteq X$ tal que $\operatorname{ext}(A)=\omega$ y $S t(A, \mathcal{U})=X$. En este trabajo a los espacios que tienen esta propiedad los abreviaremos como espacios $S C E$, esta abreviatura viene del inglés star countable extent.

Lema 3.2.1. Si $X=\bigcup\left\{Y_{n}: n<\omega\right\}$ es un espacio topológico y $Y_{n}$ es un espacio con extensión numerable para cada $n<\omega$, entonces $X$ tiene extensión numerable. 
Demostración. Supongamos que $\operatorname{ext}(X)>\omega$, entonces existe un subconjunto $D \subseteq$ $\bigcup\left\{Y_{n}: n<\omega\right\}$ no numerable, cerrado y discreto. Sin perdida de generalidad supongamos que $|D|=\omega_{1}$. Entonces, existe $m<\omega$ tal que $\left|D \cap Y_{m}\right|=\omega_{1}$ y $D \cap Y_{m}$ es un subespacio cerrado y discreto de $Y_{m}$ lo que contradice que $\operatorname{ext}\left(Y_{m}\right)=\omega$. Por lo tanto $\operatorname{ext}(X)=\omega$.

Corolario 3.2.2. Sea $X=\bigcup\left\{Y_{n}: n<\omega\right\}$ un espacio topológico y $Y_{n}$ es un espacio $S C E$ para cada $n<\omega$, entonces $X$ es $S C E$.

Demostración. Sea $\mathcal{U}$ una cubierta abierta de $X$. Para cada $n<\omega$, existe $A_{n} \subseteq Y_{n}$ con $\operatorname{ext}\left(A_{n}\right)=\omega$ y $S t\left(A_{n}, \mathcal{U}\right)=Y_{n}$. Por el Lema 3.2.1 tenemos que $A=\bigcup\left\{A_{n}: n<\kappa\right\}$ tiene extensión numerable y $S t(A, \mathcal{U})=X$.

En las sección 3.1 se demostraron las siguientes implicaciones en la clase de los espacios $T_{1}$ :

$\omega_{1}$-Lindelöf $\Rightarrow$ Extensión numerable $\Rightarrow$ Estrella numerable $\Rightarrow$ Estrella $\sigma$-compacto $\Rightarrow$ Estrella Lindelöf $\Rightarrow$ Tenuemente Lindelöf.

El siguiente resultado es relevante ya que como consecuencia de este podemos relacionar los espacios estrella Lindelöf con los espacios $S C E$.

Lema 3.2.3. Si $X$ es un espacio de Lindelöf, entonces $X$ tiene extensión numerable.

Demostración. Supongamos que $D=\left\{x_{\alpha}: \alpha<\kappa\right\} \subseteq X$ es un subespacio no numerable, cerrado y discreto. Para cada $\alpha<\kappa$, sea $U_{\alpha} \in \tau\left(x_{\alpha}, X\right)$ tal que $U_{\alpha} \cap D=$ $\left\{x_{\alpha}\right\}$, entonces $\mathcal{U}=\left\{U_{\alpha}: \alpha<\kappa\right\} \cup\{X \backslash D\}$ es una cubierta abierta de $X$ que no tiene subcubierta numerable, así $X$ no es de Lindelöf.

Corolario 3.2.4. Si $X$ es un espacio estrella Lindelöf, entonces $X$ es un espacio $S C E$.

Demostración. Sea $\mathcal{U}$ una cubierta abierta de $X$, como $X$ es estrella Lindelöf existe un subconjunto $L$ Lindelöf tal que $S t(L, \mathcal{U})=X$ y por el Lema $3.2 .3 \operatorname{ext}(L)=\omega$.

Corolario 3.2.5. Si $X$ es un espacio que tiene una familia celular no numerable y localmente finita de subconjuntos no vacíos, entonces $X$ no es un espacio $S C E$.

Demostración. Sea $\mathcal{U}=\left\{U_{\alpha}: \alpha<\kappa\right\}$ una familia no numerable, celular y localmente finita de subconjuntos no vacíos. Por el Lema 3.1.6, si elegimos $d_{\alpha} \in U_{\alpha}$ para cada $\alpha<\kappa$ el subconjunto $D=\left\{d_{\alpha}: \alpha<\kappa\right\}$ es no numerable, cerrado y discreto; así la cubierta $\mathcal{V}=\mathcal{U} \cup\{X \backslash D\}$ es una cubierta abierta sin núcleo con extensión numerable.

Este último Corolario y el Lema 1.2.6 implican el siguiente resultado: 
Corolario 3.2.6. Si $X$ es un espacio $S C E$, entonces $X$ es tenuemente Lindelöf.

A continuación daremos un ejemplo de un espacio que es tenuemente Lindelöf pero no estrella Lindelöf, más aún, este espacio tiene un subconjunto denso con extensión numerable, lo que nos muestra que la clase de los espacios $S C E$ y la clase de los espacios estrella Lindelöf mantienen una distancia considerablemente grande. Con este mismo ejemplo podemos observar que tener un subconjunto denso con una propiedad topológica $P$ no es equivalente a tener un subconjunto denso estrella $P$.

Ejemplo 3.2.7. Existe un espacio topológico Tychonoff que es $S C E$ y que no es estrella Lindelöf.

Demostración. Sean $S=\left\{\alpha+1: \alpha<\omega_{1}\right\}$ y $X=\left(\omega_{1} \times \omega\right) \cup(S \times\{\omega\})$ considerado como subespacio de $\omega_{1} \times(\omega+1)$. Notemos que $Z=\omega_{1} \times \omega$ es un subconjunto denso de $X$. Como $Z$ es $\sigma$-numerablemente compacto, se sigue que $e(Z) \leq \omega$, por lo tanto $X$ es un espacio $S C E$.

Falta ver que $X$ no es estrella Lindelöf. Consideremos la siguiente cubierta abierta:

$$
\mathcal{U}=\left\{\omega_{1} \times \omega\right\} \cup\{\{\alpha\} \times(\omega+1): \alpha \in S\} .
$$

Si $L \subseteq X$ es un subespacio Lindelöf, entonces $L$ debe ser acotado en sus primeras coordenadas, es decir, existe $\alpha_{0}<\omega_{1}$ tal que si $(\alpha, n) \in L$, entonces $\alpha \leq \alpha_{0}$. Tenemos que $L$ es ajeno a $\left\{\alpha_{0}+1\right\} \times(\omega+1)$, así $\left(\alpha_{0}+1, \omega\right) \notin S t(L, \mathcal{U})$, por lo tanto $X$ no puede ser estrella Lindelöf.

Ahora estudiaremos el comportamiento de los subespacios de los espacios $S C E$. Se darán las condiciones necesarias para que la propiedad $S C E$ sea heredada a subespacios. El primer ejemplo que daremos muestra que la propiedad $S C E$ no se hereda por subconjuntos cerrados y el segundo muestra que tampoco se hereda por subconjuntos abiertos.

Ejemplo 3.2.8. Existe un espacio $S C E$ que tiene subconjuntos cerrados que no son $S C E$.

Demostración. Si $X=\psi(A)$ es un espacio de Mrówka para alguna familia MAD $\mathcal{A}$ en $\omega$, entonces $X$ es un espacio SCE ya que $X$ es separable. Además, $\mathcal{A}$ es un subespacio no numerable, cerrado y discreto ; por lo tanto no puede ser un espacio $S C E$. Esto prueba que la propiedad $S C E$ no necesariamente es heredable a subconjunto cerrados o a subconjuntos cerrados $G_{\delta}$ o a subconjuntos nulos.

Ejemplo 3.2.9. Existe un espacio $S C E$ que tiene subconjuntos abiertos que no son $S C E$. 
Demostración. Sean $D$ un espacio no numerable y discreto y sea $X$ la compactación unipuntual de $D$, entonces $X$ es un espacio $S C E$, pero $D$ no lo es. Esto prueba que la propiedad SCE no necesariamente se hereda a subconjunto abiertos o a subespacios densos, ni siquiera a subespacios abiertos densos.

Proposición 3.2.10. Si $X$ es un espacio $S C E$ y $A \subseteq X$ es un subconjunto abierto $F_{\sigma}$, entonces $A$ es $S C E$.

Demostración. Sea $A \subseteq X$ un subespacio abierto $F_{\sigma}$, es decir, $A=\bigcup\left\{F_{n}: n<\omega\right\}$ y cada $F_{n}$ es un subconjunto cerrado en $X$. Sea $\mathcal{U}$ una cubierta abierta de $A$. Como $A$ es un subconjunto abierto en $X$ se sigue que cada elemento de la cubierta $\mathcal{U}$ es un subconjunto abierto en $X$. Para cada $n \in \omega$ consideremos la familia $\mathcal{U}_{n}=\mathcal{U} \cup\left\{X \backslash F_{n}\right\}$. Así $\mathcal{U}_{n}$ es una cubierta abierta de $X$. Como $X$ es $S C E$, para cada $n<\omega$ existe un núcleo con extensión numerable $M_{n}$ para $\mathcal{U}_{n}$.

Para cada $n<\omega$ tenemos que $F_{n} \subseteq S t\left(M_{n} \cap A, \mathcal{U}\right)$. Entonces $A=\operatorname{St}(M, \mathcal{U})$, donde $M=\bigcup\left\{M_{n} \cap A: n<\omega\right\}$. Afirmamos que $e(M) \leq \omega$, para esto es suficiente demostrar que cada $M_{n} \cap A$ tiene extensión numerable. Como el subconjunto $M_{n} \cap A=$ $\bigcup\left\{\left(M_{n} \cap F_{m}\right): m<\omega\right\}$, ya que $F_{m}$ es un subconjunto cerrado en $X$ y $e\left(M_{n}\right) \leq \omega$, tenemos que $e\left(M_{n} \cap F_{m}\right) \leq \omega$, y así $e\left(M_{n} \cap A\right) \leq \omega$. Esto prueba que $A$ es $S C E$.

Corolario 3.2.11. Si $X$ es un espacio $S C E$ y $A \subseteq X$ es un subconjunto abierto y cerrado, entonces $A$ es $S C E$.

Demostración. Sea $\mathcal{U}$ una cubierta abierta de $A$, entonces $\mathcal{V}=\mathcal{U} \cup\{X \backslash A\}$ es una cubierta abierta de $X$, entonces existe un núcleo con extensión numerable $M \subseteq X$ para $\mathcal{V}$, así $S t(M \cap A, \mathcal{U})=A$.

Corolario 3.2.12. Si $X$ es un espacio $S C E$ y $A \subseteq X$ es un subconjunto conulo, entonces $A$ es $S C E$.

Demostración. Cada subconjunto conulo es subconjunto $F_{\sigma}$.

Definición 3.2.13. Decimos que un subespacio $U$ de un espacio topológico $X$ es un dominio abierto si $U=\operatorname{int}(\operatorname{cl}(U))$.

Ejemplo 3.2.14. Existe un espacio $S C E$ que tiene subconjuntos que son dominios abiertos que no son $S C E$.

Demostración. Sea $L$ la Lindelöficación unipuntual de un subespacio no numerable discreto y sea $S$ una sucesión convergente no trivial junto con su límite. Sea $X$ el espacio cociente obtenido de $L \oplus S$ por la identificación de los puntos no aislados de 
$L$ y $S$. Como $X$ es la imagen continua de un espacio de Lindelöf, tenemos que $X$ es un espacio de Lindelöf. Por otra parte, es fácil verificar que los puntos aislados de $L$ forman un dominio abierto de $X$ que no es SCE. Por lo tanto la propiedad $S C E$ no se hereda a dominios abiertos.

Definición 3.2.15. Decimos que un subespacio $C$ de un espacio topológico $X$ es un dominio cerrado si $C=\operatorname{cl}(\operatorname{int}(C))$.

Ejemplo 3.2.16. Existe un espacio Tychonoff estrella numerable con un subespacio que es un dominio cerrado y que no es $S C E$.

Demostración. Vamos a construir recursivamente una familia MAD de subconjuntos numerables de $\omega_{1}$ de tamaño $2^{\omega}$.

Para cada $\alpha<\omega_{1}$ sean $\omega_{\alpha}=\omega \times\{\alpha\}$ y $N_{0}=\emptyset$. Ahora para cada $0<\beta \leq \omega_{1}$ sea $N_{\beta}=\bigcup\left\{\omega_{\alpha}: \alpha<\beta\right\}$.

Sea $\mathcal{A}_{1}$ una familia MAD en $N_{1}$ con $\left|\mathcal{A}_{1}\right|=\mathfrak{c}$ y $\mathcal{A}_{2}$ una familia MAD en $N_{2}$ tal que $\mathcal{A}_{2}$ extiende a $\mathcal{A}_{1}\left(\mathcal{A}_{1} \subset \mathcal{A}_{2}\right)$. Asumamos que para cada $\alpha<\beta$ hemos construido una familia MAD $\mathcal{A}_{\alpha}$ en $N_{\alpha}$ tal que si $\delta<\alpha$, entonces $\mathcal{A}_{\delta} \subset \mathcal{A}_{\alpha}$. Como $\bigcup\left\{\mathcal{A}_{\alpha}: \alpha<\beta\right\}$ es una familia casi ajena en $N_{\beta}$ podemos extenderla a una familia MAD $\mathcal{A}_{\beta}$.

Sea $\mathcal{A}=\bigcup\left\{\mathcal{A}_{\alpha}: \alpha<\omega_{1}\right\}$, entonces $\mathcal{A}$ es $\mathrm{AD}$ en $N_{\omega_{1}}$ y demostraremos que $\mathcal{A}$ es una familia MAD. Con este fin, si $A \subset N_{\omega_{1}}$ y $|A|=\omega$ se tiene que existe $\delta \in \omega_{1}$ tal que $A \subset N_{\delta}$ y $\mathcal{A}_{\delta}$ es $\mathrm{MAD}$ en $N_{\delta}$, así que existe $B \in \mathcal{A}_{\delta}$ tal que $|A \cap B|=\omega$. Por lo tanto, existe $B \in \mathcal{A}$ tal que $|A \cap B|=\omega$; así $\mathcal{A}$ es maximal.

Sea $X_{1}=\Psi(\mathcal{A})$, el primer paso es mostrar que $X_{1}$ no es un espacio SCE. Notemos que si $A \in \mathcal{A}_{\alpha+1} \backslash \mathcal{A}_{\alpha}$, entonces $\left|A \cap N_{\alpha}\right|<\omega$, pues si esto no fuera cierto por la maximalidad de $\mathcal{A}_{\alpha}$ existiría un $B \in \mathcal{A}_{\alpha} \subset \mathcal{A}_{\alpha+1}$ tal que $|A \cap B|=\omega$, pero esto no es posible ya que $\mathcal{A}_{\alpha+1}$ es casi ajena. Puesto que $\{A\} \cup A$ es una vecindad abierta de $A$ en $N_{\alpha+1} \cup\{A\}$ se sigue que $\{A\} \cup\left(A \backslash\left(A \cap N_{\alpha}\right)\right)$ es una vecindad abierta de $A$.

Para cada $A \in \mathcal{A}$ definimos:

(1) $U_{A}=\{A\} \cup\left(A \backslash N_{\alpha}\right)$, si $A \in \mathcal{A}_{\alpha+1} \backslash \mathcal{A}_{\alpha}$ para algún $\alpha<\omega_{1} \mathrm{y}$

(2) $U_{A}=\{A\} \cup A$ en otro caso.

Ahora:

$\mathcal{U}=\left\{U_{A}: A \in \mathcal{A}\right\} \cup\left\{\{p\}: p \in N_{\omega_{1}}\right\}$, es una cubierta abierta de $X_{1}$. 
Sea $M \subset X_{1}$ un subespacio con $e(M)=\omega$. Notemos que $M \cap \mathcal{A}$ es un subconjunto cerrado y discreto en $M$, se sigue que $M \cap \mathcal{A}$ es numerable. Como $M \backslash \bigcup\left\{U_{A}: A \in\right.$ $M \cap \mathcal{A}\} \subset N_{\omega_{1}}$ es cerrado y discreto en $M$, tenemos que $M$ es en efecto numerable. Tomamos $\alpha<\omega_{1}$ tal que $M \cap N_{\omega_{1}} \subset N_{\alpha}$. Veamos que $\mathcal{A}_{\alpha+1} \backslash \mathcal{A}_{\alpha}$ es no numerable, para ello supongamos lo contrario, es decir, $\mathcal{A}_{\alpha+1} \backslash \mathcal{A}_{\alpha}=\left\{A_{\delta}: \delta<\omega\right\}$ y elegimos $x_{\delta} \in A_{\delta} \backslash \bigcup\left\{A_{\beta}: \beta<\delta\right\}$ para cada $\delta<\omega$, entonces $C=\left\{x_{\delta}: \delta<\omega\right\}$ es un subconjunto numerable y $\mathcal{A}_{\alpha+1} \cup\{C\}$ es una familia casi ajena en $N_{\alpha+1}$ lo que contradice que $\mathcal{A}_{\alpha+1}$ es maximal. Por lo tanto podemos escoger $A \in \mathcal{A}_{\alpha+1} \backslash\left(\mathcal{A}_{\alpha} \cup(M \cap \mathcal{A})\right)$. Por construcción, $U_{A} \cap M=\emptyset$, así $A \notin S t(M, \mathcal{U})$. Por lo tanto $\mathcal{U}$ no tiene un núcleo con extensión numerable.

Ahora sea $\mathcal{A}^{\prime}$ una familia MAD en $\omega$ con $\left|\mathcal{A}^{\prime}\right|=2^{\omega}$ y sea $X_{2}=\Psi\left(\mathcal{A}^{\prime}\right)$ el espacio de Mrówka asociado a $\mathcal{A}^{\prime}$. Sea $i: \mathcal{A} \rightarrow \mathcal{A}^{\prime}$ una biyección y sea $X$ el espacio cociente obtenido de $X_{1} \oplus X_{2}$ al identificar $A$ con $i(A)$. Sea $q: X_{1} \oplus X_{2} \rightarrow X$ el mapeo cociente. Consideramos a $X_{1}$ y a $X_{2}$ como subespacios de $X$ con $X_{1} \cap X_{2}=\mathcal{A}$.

$$
X=\omega_{1} \cup\{(A, i(A)): A \in \mathcal{A}\} \cup \omega .
$$

Tenemos que $X_{1}$ es un dominio cerrado en $X$. Para ver que $X$ es estrella numerable, sea $\mathcal{U}$ una cubierta abierta de $X$. Como $X_{2}$ es separable, existe un subconjunto numerable $K \subseteq X_{2}$ tal que $X_{2} \subseteq S t(K, \mathcal{U})$; en particular $\mathcal{A} \subset S t(K, \mathcal{U})$. Sea $L=X_{1} \backslash S t(K, \mathcal{U})$ y notemos que $L$ es finito. De otra forma, podemos tomar $B \subset X_{1} \backslash S t(K, \mathcal{U}) \subset \omega \times \omega_{1}$ con $|B|=\omega$ y por la maximalidad de $\mathcal{A}$ existe un $A \in \mathcal{A}$ tal que $|A \cap B|=\omega$. Como $A \in S t(K, \mathcal{U})$, existe $F \subset A$ finito tal que $A \backslash F \subset S t(K, \mathcal{U})$, pero esto implica que $B \cap S t(K, \mathcal{U}) \neq \emptyset$ lo cual no es posible. Por lo tanto $K \cup L$ es un núcleo numerable para $\mathcal{U}$. Así $X$ es estrella numerable.

A continuación estudiaremos las imágenes continuas de espacios $S C E$ y proporcionaremos condiciones para que la propiedad $S C E$ se conserve bajo funciones continuas.

Lema 3.2.17. Si $X$ es un espacio topológico con extensión numerable y $f: X \rightarrow Y$ es una función continua suprayectiva, entonces $\operatorname{ext}(Y)=\omega$.

Demostración. Supongamos que $e(Y)>\omega$, entonces existe $D=\left\{d_{\alpha}: \alpha<\omega_{1}\right\} \subseteq Y$ cerrado y discreto; para cada $\alpha<\omega_{1}$ escogemos $c_{\alpha} \in f^{-1}\left[d_{\alpha}\right]$ y sea $C=\left\{c_{\alpha}: \alpha \in \omega_{1}\right\}$. Afirmamos que $C$ es cerrado y discreto. Para ver que es discreto, notemos que para cada $\alpha \in \omega_{1}$, existe $V_{\alpha}$ subconjunto abierto de $Y$ tal que $V_{\alpha} \cap D=\left\{d_{\alpha}\right\}$, entonces $c_{\alpha} \in f^{-1}\left[V_{\alpha}\right]$ y $f^{-1}\left[V_{\alpha}\right] \cap C=\left\{c_{\alpha}\right\}$, por lo que $C$ es discreto en $X$.

Para ver que $C$ es cerrado, sea $z \in X \backslash C$, hay dos posibilidades:

(1) $f(z) \notin D$, entonces $f(z) \in Y \backslash D$, así $x \in f^{-1}[Y \backslash D]$ que es un subconjunto 
abierto en $X$ y $f^{-1}[Y \backslash D] \cap C=\emptyset$.

(2) $f(z) \in D$, es decir, $f(z)=d_{\alpha}$ y notemos que $z \neq c_{\alpha}$, entonces existe una vecindad $V_{\alpha}$ de $d_{\alpha}$ tal que $V_{\alpha} \cap D=\left\{d_{\alpha}\right\}$, por lo que $f^{-1}\left[V_{\alpha}\right] \cap C=\left\{c_{\alpha}\right\}$; así $f^{-1}\left[V_{\alpha}\right] \backslash\left\{c_{\alpha}\right\}$ es una vecindad abierta de $z$ ajena a $C$.

Estos hechos contradicen que $e(X)$ es numerable, por lo tanto lo supuesto es falso, así $e(Y)=\omega$.

El siguiente resultado es consecuencia del Lema 3.2.17 y de la Proposición 3.1.14.

Corolario 3.2.18. La propiedad $S C E$ se preserva bajo imágenes continuas.

Definición 3.2.19. Sean $X, Y$ espacios topológicos. Una función $f: X \rightarrow Y$ se llama función perfecta si es continua, cerrada, suprayectiva y $f^{-1}[\{y\}]$ es compacto para cada $y \in Y$.

Lema 3.2.20. Sean $X, Y$, espacios topológicos con $e(Y)=\omega$ y $f: X \rightarrow Y$ una función perfecta. Entonces $e(X)=\omega$.

Demostración. Sea $f: X \rightarrow Y$ una función perfecta, donde $Y$ es un espacio con extensión numerable. Fijemos un subespacio cerrado y discreto $M \subseteq X$. Primero verificamos que $f[M]$ es un subespacio discreto en $Y$. Dado $p \in f[M]$, el conjunto $A=M \backslash f^{-1}[\{p\}]$ es un conjunto cerrado en $X$ ya que $M$ es un subconjunto cerrado y discreto en $X$. Por lo tanto $Y \backslash f[A]$ es un subconjunto abierto en $Y$ tal que $(Y \backslash f[A]) \cap f[M]=\{p\}$. Con esto tenemos que $f[M]$ es discreto en $Y$, pero también es cerrado, pues $f$ es una función perfecta. Por lo tanto $f[M]$ es numerable.

Por otra parte, ya que $f$ es una función perfecta y $M$ es cerrado, entonces $f_{\left.\right|_{M}}: M \rightarrow$ $f[M]$ es una función perfecta. Así para cada $p \in f[M]$, se tiene que $\left(f_{\left.\right|_{M}}\right)^{-1}[\{p\}]$ es compacto en $M$ y por lo tanto es finito. Finalmente como $M=\bigcup\left\{\left(f_{\left.\right|_{M}}\right)^{-1}[\{p\}]: p \in f[M]\right\}$, tenemos que $|M| \leq \omega$, esto prueba que $e(X)=\omega$.

Lema 3.2.21. Sean $X, Y$ espacios topológicos con $Y$ un espacio $S C E$. Si $f: X \rightarrow Y$ es una función perfecta y abierta, entonces $X$ es un espacio $S C E$.

Demostración. Sea $\mathcal{U}$ una cubierta abierta de $X$. Para cada $y \in Y$ existe una subfamilia finita $\mathcal{U}_{y} \subset \mathcal{U}$ tal que $f^{-1}[\{y\}] \subset \bigcup \mathcal{U}_{y}$. Podemos asumir que para cada $U \in \mathcal{U}_{y}$, $U \cap f^{-1}[\{y\}] \neq \emptyset$. Sea $V_{y}=\left(Y \backslash f\left[X \backslash \bigcup \mathcal{U}_{y}\right]\right) \cap \bigcap\left\{f[U]: U \in \mathcal{U}_{y}\right\}$, así $V_{y}$ es un subconjunto abierto, ya que $\mathcal{U}_{y}$ es finita y $f$ es una función abierta y cerrada.

La condición $U \cap f^{-1}[\{y\}] \neq \emptyset$ y el hecho de que $f^{-1}[\{y\}] \subset \bigcup \mathcal{U}_{y}$ implican que $y \in V_{y}$. Esto prueba que la familia $\mathcal{V}=\left\{V_{y}: y \in Y\right\}$ es una cubierta abierta de $Y$. Entonces 
existe un subconjunto $N$ en $Y$ con $e(N)=\omega$ y tal que $S t(N, \mathcal{V})=Y$. Sea $M=f^{-1}[N]$. Por el Lema 3.2.20 tenemos que $M$ tiene extensión numerable.

Ahora mostremos que $M$ es un núcleo para la cubierta $\mathcal{U}$. Sea $x \in X$, entonces existe $y \in Y$ tal que $f(x) \in V_{y}$ y $V_{y} \cap N \neq \emptyset$. Como $V_{y} \subset Y \backslash f\left[X \backslash \bigcup \mathcal{U}_{y}\right]$ y $f^{-1}\left[V_{y}\right] \subset \bigcup \mathcal{U}_{y}$, entonces $x \in U$ para algún $U \in \mathcal{U}_{y}$. Como $V_{y} \subset f[U]$ y $V_{y} \cap N \neq \emptyset$, tenemos que $U \cap M \neq \emptyset$, así $x \in S t(M, \mathcal{U})$. Por lo tanto $X$ es un espacio $S C E$.

A continuación mostraremos que la propiedad $S C E$ no se preserva bajo imágenes inversas de funciones perfectas. Para ello necesitamos definir un espacio importante en diversas áreas de la Topología y algunas propiedades básicas relacionadas con funciones perfectas. $\mathrm{Si}(X, \tau)$ es un espacio topológico, entonces se define el Duplicado de Alexandroff como $A(X)=X \times\{0,1\}$ con la topología generada por la siguiente base $\mathcal{B}$ :

1) Para cada $x \in X,\{(x, 1)\} \in \mathcal{B}$;

2) Para cada $x \in X$ y $U \in \tau(x, X),(U \times\{0\}) \cup((U \backslash\{x\}) \times\{1\}) \in \mathcal{B}$.

Lema 3.2.22. Sea $X$ un espacio topológico y $A(X)=X \times\{0,1\}$ el duplicado de Alexandroff de $X$. Entonces la función $f: A(X) \rightarrow X$ definida como $f(x, i)=x$, para $i=0,1$, es una función perfecta.

Demostración. (1) Tenemos que $f^{-1}[\{x\}]=\{(x, i): i=0,1\}=\{(x, 0),(x, 1)\}$; así $f^{-1}[\{x\}]$ es compacto para cada $x \in X$.

(2) Sea $U \subseteq X$ un subconjunto abierto, entonces $f^{-1}[U]=(U \times\{0\}) \cup(U \times\{1\})$ que es un subconjunto abierto en $A(X)$; por lo tanto $f$ es una función continua.

(3) Para cada $B \subseteq X$, identificamos $B$ con $B \times\{0\} \subseteq A(X)$. Sea $\pi: A(X) \rightarrow$ $X_{0} \equiv X$ la proyección y $C \subseteq A(X)$ un subconjunto cerrado de $A(X)$. Se define $C_{i}=C \cap(X \times\{i\})$ para $i \in\{0,1\}$. Entonces $\pi[C]=\pi\left[C_{1} \cup C_{0}\right]=\pi\left[C_{1}\right] \cup C_{0}$. Notamos primero que $X \times\{1\}$ es abierto en $A(X)$ y por tanto $X \times\{0\}$ es cerrado. Así, $C_{0}=X_{0} \cap C$ es cerrado en $A(X)$.

Para probar que $\pi[C]$ es cerrado, sea $p \in \operatorname{cl}(\pi[C])$; se requiere demostrar que $p \in \pi[C]$. Vamos a ver que $(p, 0) \in \operatorname{cl}(C)=C$. Sea $W \subseteq \tau((p, 0), A(X))$, entonces existe $V \in \tau(X)$ tal que $p \in V$ y $(V \times\{0\}) \cup((V \backslash\{p\}) \times\{1\}) \subseteq W$. Como $V \cap \pi[C] \neq \emptyset$, tenemos que existe $(x, i) \in C$ tal que $\pi(x, i)=(x, 0) \in(V \times\{0\}) \cap C$. Si $i=0$, $(x, 0) \in W \cap C$. Si $i=1$ y $x \neq p$, entonces $(x, 1) \in W \cap C$. Si $i=1$ y $x=p$, entonces $(p, 0) \in W \cap C$. En cualquier caso $W \cap C \neq \emptyset$. Por lo tanto $(p, 0) \in \operatorname{cl}(C)=C$, se sigue que $p=\pi((p, 0)) \in \pi[C]$. 


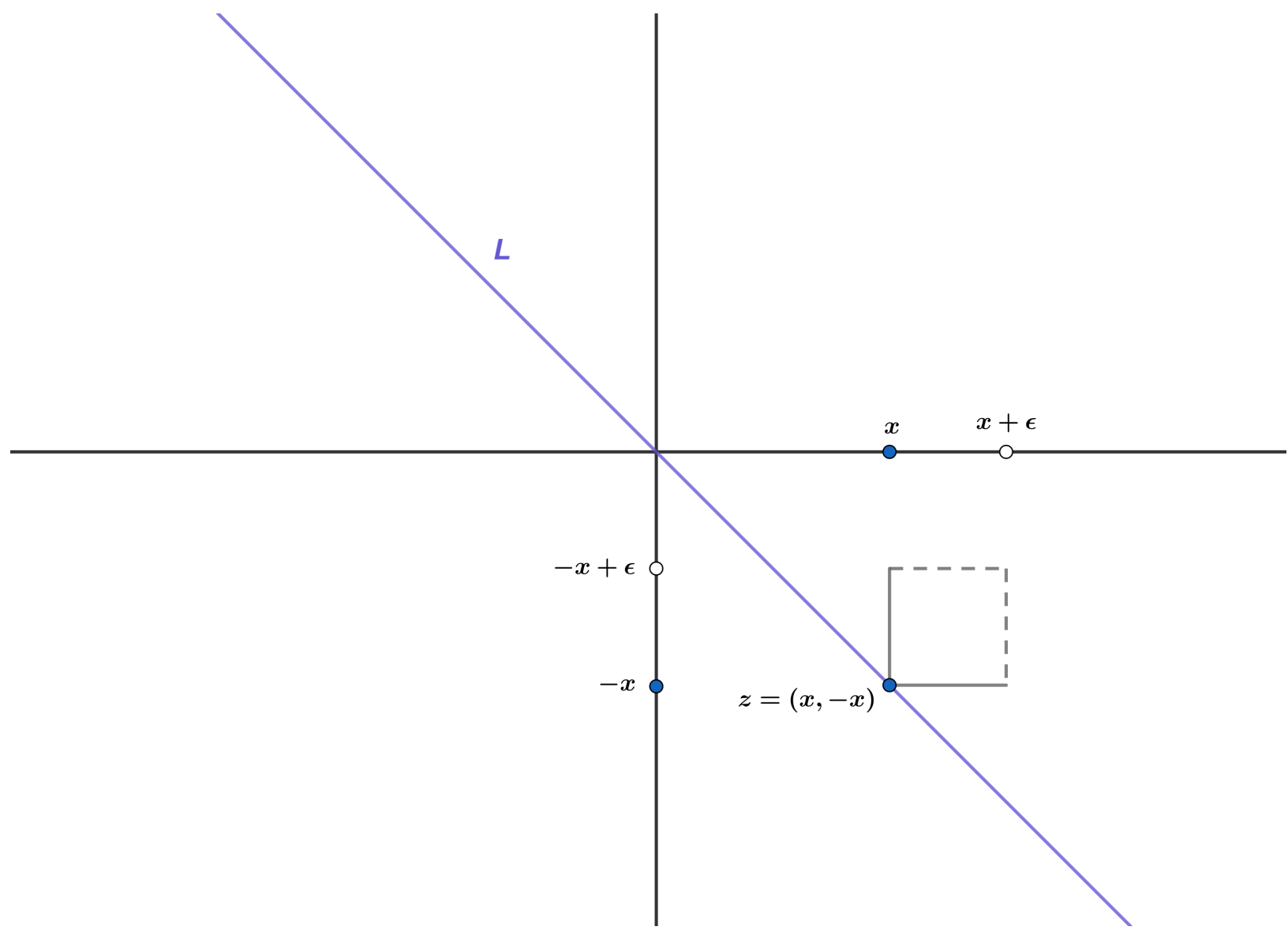

Figure 3.1: El cuadrado de la recta de Sorgenfrey

Lema 3.2.23. Sea $S^{2}$ el cuadrado de la Recta de Sorgenfrey, entonces el conjunto $L=\left\{(x,-x) \in S^{2}: x \in S\right\}$ es un subconjunto cerrado y discreto.

Demostración. Sean $z=(x,-x) \in L$ y $\epsilon>0$, entonces $z \in[x, x+\epsilon) \times[-x,-x+\epsilon)$ y tenemos que $([x, x+\epsilon) \times[-x,-x+\epsilon)) \cap L=\{z\}$, por lo que $L$ es un subconjunto discreto de $S^{2}$.

Ejemplo 3.2.24. La propiedad $S C E$ no se preserva bajo imágenes inversas de funciones perfectas.

Demostración. Sea $S^{2}$ el cuadrado de la Recta de Sorgenfrey. Como $S^{2}$ es separable, entonces también es un espacio $S C E$. Por el Lema 3.2.22, $f: A\left(S^{2}\right) \longrightarrow S^{2}$ definida como $f(p, i)=p$, para $i=0,1$, es una función perfecta. Consideremos el subconjunto $L=\left\{(x,-x) \in S^{2}: x \in S\right\}$; por el Lema 3.2.23 tenemos que $L$ es un subconjunto cerrado y discreto en $S^{2}$. 
Para cada $p \in S^{2} \backslash L$ definimos $U_{p}=U \times\{0,1\}$, donde $U \in \tau\left(p, S^{2}\right)$ y $U \cap L=\emptyset$. Similarmente para cada $q \in L$ definimos $U_{q}=(U \times\{0,1\}) \backslash\{(q, 1)\}$, donde $U \in \tau\left(q, S^{2}\right)$ y $U \cap L=\{q\}$.

Notemos que para cualquier $s \in S^{2}$ se tiene que $U_{s} \cap(L \times\{1\})=\emptyset$. Esto implica en particular que $L \times\{1\}$ es un subconjunto cerrado en $A\left(S^{2}\right)$, pero todos los puntos de $L \times\{1\}$ son aislados. Por lo tanto $L \times\{1\}$ es un subconjunto abierto, cerrado, discreto y no numerable de $A\left(S^{2}\right)$; se sigue que $L \times\{1\}$ no es un espacio $S C E$. Como la propiedad $S C E$ se hereda a subconjuntos abiertos y cerrados tenemos que el espacio $A\left(S^{2}\right)$ no es un espacio $S C E$.

Proposición 3.2.25. Si para un espacio topológico $X$ tenemos que $A(X)$ es un espacio $S C E$, entones $e(X) \leq \omega$.

Demostración. Supongamos que $e(X)>\omega$, entones existe un subconjunto cerrado, discreto y no numerable $D$ en $X$. Para cada $z \in X \backslash D$ definimos $U_{x}=U \times\{0,1\}$, donde $U \in \tau(x, X)$ y $U \cap D=\emptyset$. Similarmente para cada $d \in D$ definimos $U_{d}=$ $(U \times\{0,1\}) \backslash\{(d, 1)\}$, donde $U \in \tau(d, X)$ y $U \cap D=\{d\}$.

Notemos que para cualquier $x \in X$ se tiene que $U_{x} \cap(D \times\{1\})=\emptyset$. Esto implica que $D \times\{1\}$ es un subconjunto cerrado en $A(X)$, pero todos los puntos de $D \times\{1\}$ son aislados. Por lo tanto $D \times\{1\}$ es un subconjunto abierto, cerrado, discreto y no numerable de $A(X)$; se sigue que $D \times\{1\}$ no es un espacio $S C E$. Como la propiedad $S C E$ se hereda a subconjuntos abiertos y cerrados tenemos que el espacio $A(X)$ no es un espacio $S C E$.

Proposición 3.2.26. Si $e(X) \leq \omega$, entonces $e(A(X)) \leq \omega$.

Demostración. Observemos que $A(X)$ es imagen inversa de $X$ de una función perfecta y como $e(X)=\omega$, por el Lema 3.2.20, obtenemos el resultado.

De estos últimos dos resultados podemos inferir que en la clase de los espacios que son duplicados de Alexandroff, las propiedades extensión numerable y $S C E$ son equivalentes. Así tenemos el sisguiente corolario.

Corolario 3.2.27. Para cualquier espacio topológico $X$ tenemos que $e(A(X)) \leq \omega$ si y sólo si $A(X)$ es un espacio $S C E$.

Definición 3.2.28. Sea $(X, \tau)$ un espacio topológico. Se llama número de Lindelöf de $X$ a:

$l(X)=\min \{\kappa:$ cada cubierta abierta de $X$ tiene una subcubierta de cardinalidad $\leq \kappa\}$. 
Definición 3.2.29. Sea $(X, \tau)$ un espacio topológico. Se llama densidad de $X$ a:

$$
d(X)=\min \{|D|: \operatorname{cl}(D)=X\}+\omega .
$$

En el Corolario 3.2.27 se demostró que el duplicado de Alexandroff de un espacio topológico tiene extensión numerable si y sólo si es un espacio $S C E$. Considerando que cada espacio es la imagen de una función continua de un duplicado de Alexandroff, es claro que en el Corolario 3.2.27 no podemos sustituir $e(A(X)) \leq \omega$ por $l(A(X)) \leq \omega$ o por $d(A(X)) \leq \omega$.

En la sección 3.2.1 se demostró que cada espacio $S C E$ es tenuemente Lindelöf. A continuación se muestra que la implicación recíproca no se cumple.

Ejemplo 3.2.30. Existe un P-espacio de Tychonoff tenuemente Lindelöf que no es $S C E$.

Demostración. El espacio es el del Ejemplo 3.1.30. Sean $M \subset X=\omega_{1} \cup \mathcal{A}$, con $e(M)=\omega$ y $\mathcal{U}$ una cubierta abierta de $M$. Puesto que $\mathcal{A}$ es cerrado y discreto, se sigue que $M \cap \mathcal{A}$ es numerable, y por tanto existe una subfamilia numerable $\mathcal{V} \subseteq \mathcal{U}$ tal que $M \cap \mathcal{A} \subseteq \bigcup \mathcal{V}$. Como el subconjunto $B=M \backslash \bigcup \mathcal{V}$ de $\omega_{1}$ es cerrado y discreto, y $e(M)=\omega$, entonces $B$ es numerable. Por tanto existe una subfamilia $\mathcal{W} \subseteq \mathcal{U}$ que cubre a $B$ y $\mathcal{V} \cup \mathcal{W}$ es una subcubierta numerable de $M$ de $\mathcal{U}$.

Por lo anterior y por el Teorema 3.1.13 se tiene que en el espacio $X$ un subespacio es de Lindelöf si y sólo si $e(X)=\omega$. Como $X$ no es estrella Lindelöf, se sigue que $X$ no es $S C E$.

Ahora veremos que la propiedad $S C E$ no es estable bajo productos finitos, incluso esta propiedad no se conserva bajo productos con espacios con propiedades más fuertes que $S C E$.

Como consecuencia del Lema 3.2.21 tenemos que la propiedad $S C E$ es compactamente productiva, es decir:

Proposición 3.2.31. Si $X$ es un espacio $S C E$ y $K$ es un espacio compacto, entonces $X \times K$ es un espacio $S C E$.

Demostración. Basta tomar la función $f: X \times K \rightarrow X$, dada por $f((x, k))=x$.

Corolario 3.2.32. Si $X$ es un espacio $S C E$ y $Y$ es un espacio $\sigma$-compacto, entonces $X \times Y$ es un espacio $S C E$. 
Demostración. Supongamos que $Y=\bigcup\left\{K_{n}: n \in \omega\right\}$, donde $K_{n}$ es un subespacio compacto para cada $n \in \omega$. Entonces $X \times Y=\bigcup\left\{X \times K_{n}: n \in \omega\right\}$ y por la Proposición 3.2.31 cada $X \times K_{n}$ es un espacio $S C E$. Ahora por el Corolario 3.2.2 tenemos que $X \times Y$ es un espacio $S C E$.

A continuación exhibimos un ejemplo que muestra que si en la Proposición 3.2.31 debilitamos alguna hipótesis podemos obtener un producto que no es $S C E$.

Ejemplo 3.2.33. Existe un espacio numerablemente compacto y un espacio de Lindelöf tal que su producto no es un espacio $S C E$.

Demostración. Sea $Z=\omega_{1} \times L$, donde $L$ es la Lindelöficación unipuntual de $\omega_{1}$. Sea:

$$
\mathcal{U}=\left\{\left(\alpha, \omega_{1}\right) \times\{\alpha\}: \alpha<\omega_{1}\right\} .
$$

Es fácil ver que el conjunto $U=\{(\alpha, \beta) \in Z: \beta \geq \alpha\}$ es un subconjunto abierto de $Z$. Así $\mathcal{V}=\mathcal{U} \cup\{U\}$ es una partición abierta de $Z$. Entonces también es una partición de subconjuntos abiertos y cerrados, por lo que $\mathcal{V}$ es una familia discreta no numerable. Por el Corolario 3.2.5, $Z$ no es un espacio $S C E$.

Definición 3.2.34. Decimos que $A \subseteq \mathbb{R}$ es totalmente imperfecto si no contiene una copia del conjunto de Cantor.

Observación 3.2.35. Notemos que si $A \subseteq \mathbb{R}$ es un subconjunto totalmente imperfecto y si $F \subseteq \mathbb{R}$ es un subconjunto cerrado y no numerable, entonces $F \nsubseteq A$. El siguiente Teorema lo demostró Bernstein y puede ver la demostración en [15].

Teorema 3.2.36. Existe $A \subset \mathbb{R}$ tal que $|A|=|\mathbb{R} \backslash A|=2^{\omega}$ y tanto $A$ como $\mathbb{R} \backslash A$ son conjuntos totalmente imperfectos.

Ejemplo 3.2.37. Existen dos espacios de Lindelöf tal que su producto no es un espacio $S C E$.

Demostración. Sea $A \subseteq \mathbb{R}$ un subconjunto que cumple las condiciones del Teorema 3.2.36. Tomemos el duplicado de Alexandroff de $\mathbb{R}, A(\mathbb{R})=\mathbb{R} \times\{0,1\}$ y sean:

$$
X_{1}=(A \times\{0\}) \cup(\mathbb{R} \times\{1\}) \text { y } X_{2}=((\mathbb{R} \backslash A) \times\{0\}) \cup(\mathbb{R} \times\{1\})
$$

subespacios de $A(\mathbb{R})$.

Sean $\mathcal{B}$ la colección de los intervalos abiertos de $\mathbb{R}$ y $\mathcal{B}_{x}=\{B \in \mathcal{B}: x \in B\}$. La colección $\mathcal{A}_{1}=\left\{U_{x} \times(\{0,1\} \backslash\{(x, 1)\}): x \in A\right.$ y $\left.U_{x} \in \mathcal{B}_{x}\right\} \cup\{\{(x, 1)\}: x \in \mathbb{R}\}$ es una base para el subconjunto $X_{1}$; y la colección $\mathcal{A}_{2}=\left\{U_{x} \times(\{0,1\} \backslash\{(x, 1)\}): x \in\right.$ $\mathbb{R} \backslash A$ y $\left.U_{x} \in \mathcal{B}_{x}\right\} \cup\{\{(x, 1)\}: x \in \mathbb{R}\}$ es una base para el subconjunto $X_{2}$. 
Ahora veamos que $X_{1}$ y $X_{2}$ son espacios de Lindelöf. Sea $\mathcal{U}$ una cubierta abierta de $X_{1}$ por subconjuntos de $\mathcal{A}_{1}$. Podemos asumir que:

$$
\mathcal{U}=\left\{\left(U_{x} \times\{0,1\}\right) \backslash\{(x, 1)\}: x \in F\right\} \cup\{\{(x, 1)\}: x \in G\}
$$

donde $F, G \subseteq \mathbb{R}$. Notemos que $\left\{U_{x}: x \in F\right\}$ es una cubierta abierta de $A$. Como $A \subseteq \mathbb{R}$, existe un subconjunto numerable $F_{0} \subseteq F$ tal que $A \subseteq \bigcup\left\{U_{x}: x \in F_{0}\right\}$. Definimos $U=\bigcup\left\{U_{x}: x \in F_{0}\right\}$. Entonces $\mathbb{R} \backslash U$ es un subconjunto cerrado. Como $\mathbb{R} \backslash A$ es totalmente imperfecto, por la Observación 3.2.35 tenemos que $\mathbb{R} \backslash U$ es un subconjunto numerable. Para cada $x \in F_{0} \cup(\mathbb{R} \backslash U)$ fijamos $W_{x} \in \mathcal{U}$ tal que $(x, 1) \in W_{x}$. Sea

$$
\mathcal{V}=\left\{\left(U_{x} \times\{0,1\}\right) \backslash\{(x, 1)\}: x \in F_{0}\right\} \cup\left\{W_{x}: x \in F_{0} \cup(\mathbb{R} \backslash U)\right\} .
$$

Así tenemos que $\mathcal{V}$ es una subfamilia numerable de $\mathcal{U}$. Ahora veamos que $\mathcal{V}$ también cubre a $\mathbb{R} \times\{1\}$. Tomemos $(t, 1) \in \mathbb{R} \times\{1\}$ y asumamos que $t \notin F_{0} \cup(\mathbb{R} \backslash U)$, entonces $t \in U=\bigcup\left\{U_{x}: x \in F_{0}\right\}$, así existe $x \in F_{0}$ tal que $t \in U_{x}$. Como $t \neq x$ tenemos que $(t, 1) \in\left(U_{x} \times\{0,1\}\right) \backslash\{(x, 1)\} \in \mathcal{V}$. Por lo tanto $X_{1}$ es un espacio de Lindelöf. Se demuestra similarmente que $X_{2}$ también es un espacio de Lindelöf.

Para ver que $X_{1} \times X_{2}$ no es un espacio $S C E$, consideremos la diagonal $\triangle=\{(p, p)$ : $p \in A(\mathbb{R})\}$ de $A(\mathbb{R}) \times A(\mathbb{R})$. Como $\triangle$ es un subconjunto cerrado de $A(\mathbb{R}) \times A(\mathbb{R})$, tenemos que $D=\triangle \cap\left(X_{1} \times X_{2}\right)$ es un subconjunto cerrado en $X_{1} \times X_{2}$. Ya que $D=\{(p, p): p \in \mathbb{R} \times\{1\}\}$, tenemos que cada elemento de $D$ es aislado en $X_{1} \times X_{2}$, así $D$ es un subconjunto abierto, cerrado, discreto y no numerable, por lo que no puede ser un espacio $S C E$. Como los espacios $S C E$ se heredan a subespacios abierto y cerrados, tenemos que $X_{1} \times X_{2}$ no es un espacio $S C E$. 


\section{Capítulo 4}

\section{P-espacios y espacios de Moore.}

\subsection{P-espacios}

En el Capítulo 2 se demostró que cada espacio topológico $X$ que es $T_{1}$ con extensión numerable es estrella numerable. En este capítulo mostraremos que en algunas clases de espacios topológicos ser estrella Lindelöf y tener extensión numerable son propiedades equivalentes.

Definición 4.1.1. Si $X$ es un espacio topológico y $A \subseteq X$, decimos que una familia $\mathcal{U}$ es una expansión abierta de $A$ si $\mathcal{U}=\left\{U_{a}: a \in A\right\}$ y $U_{a} \in \tau(a, X)$ para cada $a \in A$.

Definición 4.1.2. Una colección $\mathcal{F}$ de subconjuntos de un espacio $X$ se llama punto numerable si cada $x \in X$ pertenece a lo más a un número numerable de elementos de la colección $\mathcal{F}$.

Definición 4.1.3. Dado un cardinal infinito $\kappa$, decimos que un espacio topológico $X$ es débilmente $\kappa$-metaLindelöf si para cada subconjunto cerrado y discreto $D \subseteq X$ con $|D|=\kappa$ podemos encontrar un subconjunto $D^{\prime} \subseteq D$ tal que $\left|D^{\prime}\right|=\kappa$ y $D^{\prime}$ tiene una expansión abierta punto numerable.

Definición 4.1.4. Dado un cardinal infinito $\kappa$, decimos que un espacio topológico $X$ es débilmente $\kappa$-Hausdorff por colecciones si para cada subconjunto cerrado y discreto $D \subseteq X$ con $|D|=\kappa$ podemos encontrar un subconjunto $D^{\prime} \subseteq D$ tal que $\left|D^{\prime}\right|=\kappa$ y $D^{\prime}$ tiene una expansión abierta ajena.

Proposición 4.1.5. Cada espacio débilmente $\kappa$-Hausdorff por colecciones es débilmente $\kappa$-metaLindelöf.

Demostración. Cada expansión abierta disjunta es una expansión abierta punto numerable. 
Lema 4.1.6. Sea $X$ un espacio topológico con un subconjunto cerrado y discreto no numerable $E$ que tiene una expansión abierta punto numerable, entonces $X$ no es estrella numerable.

Demostración. Supongamos que $X$ es estrella numerable y tomemos una expansión abierta punto numerable $\mathcal{V}=\left\{V_{d}: d \in E\right\}$ del subconjunto $E$ tal que $V_{d} \cap E=\{d\}$ para cada $d \in E$. La familia $\mathcal{V}^{\prime}=\mathcal{V} \cup\{X \backslash E\}$ es una cubierta abierta de $X$, así que existe un subconjunto numerable $A \subseteq X$ tal que $S t\left(A, \mathcal{V}^{\prime}\right)=X$; como $\mathcal{V}^{\prime}$ es punto numerable, podemos encontrar un conjunto numerable $B \subseteq E$ tal que para cada $d \in E$, si $V_{d} \cap A \neq \emptyset$, entonces $d \in B$. Tomamos algún $d \in E \backslash B$ y observemos que $d \notin \operatorname{St}\left(A, \mathcal{V}^{\prime}\right)=X$, lo cual es una contradicción.

Corolario 4.1.7. Para cada espacio débilmente $\omega_{1}$-metaLindelöf $X$ las siguientes condiciones son equivalentes:

(i) el espacio $X$ es estrella numerable;

(ii) $\operatorname{ext}(X) \leq \omega$.

Demostración. (i) $\Rightarrow$ (ii). Supongamos que $D$ es un subconjunto discreto y cerrado tal que $|D|=\omega_{1}$. Como $X$ es débilmente $\omega_{1}$-metaLindelöf podemos encontrar un subconjunto $D^{\prime} \subseteq D$ con $\left|D^{\prime}\right|=\omega_{1}$ que tiene una expansión punto numerable. Por el Lema 4.1.6 se tiene que $X$ no es estrella numerable.

$($ ii $) \Rightarrow(i)$ Es el Corolario 2.1.8.

Corolario 4.1.8. Si un espacio $X$ es débilmente $\omega_{1}$-Hausdorff por colecciones, entonces $X$ es estrella numerable si y sólo si $\operatorname{ext}(X) \leqslant \omega$.

Demostración. Es inmediato del Corolario 4.1.7.

Lema 4.1.9. Si $X$ es un $P$-espacio regular y $D=\left\{d_{\alpha}: \alpha \in \omega_{1}\right\}$ es un subconjunto cerrado y discreto, entonces existe una expansión abierta ajena dos a $\operatorname{dos} \mathcal{U}=\left\{U_{\alpha}\right.$ : $\left.\alpha \in \omega_{1}\right\}$ de $D$.

Demostración. Como $X$ es un espacio regular para $d_{0}$ y $D \backslash\left\{d_{0}\right\}$, existen subconjuntos abiertos ajenos $U_{0}$ y $V_{0}$ tales que $d_{0} \in U_{0}$ y $D \backslash\left\{d_{0}\right\} \subseteq V_{0}$. Al haber elegido subconjuntos abiertos ajenos $U_{\beta}$ y $V_{\beta}$ para cada $\beta<\alpha<\omega_{1}$ tales que $d_{\beta} \in U_{\beta},\left\{d_{\gamma}: \gamma>\beta\right\} \subseteq$ $V_{\beta}$ y $U_{\beta}, V_{\beta} \subseteq V_{\xi}$ para cada $\xi<\beta<\alpha$, por ser $X$ un $P$-espacio podemos escoger subconjuntos abiertos ajenos $U_{\alpha}$ y $V_{\alpha}$ tales que $d_{\alpha} \in U_{\alpha},\left\{d_{\gamma}: \gamma>\alpha\right\} \subseteq V_{\alpha}$ y $U_{\alpha}, V_{\alpha} \subseteq$ $\bigcap\left\{V_{\beta}: \beta<\alpha\right\}$. Con esta contrucción tenemos que $\mathcal{U}=\left\{U_{\alpha}: \alpha \in \omega_{1}\right\}$ es una expansión abierta ajena dos a dos de $D$. 
Corolario 4.1.10. Un $P$-espacio $X$ regular es estrella numerable si y sólo $e(X) \leq \omega$.

Demostración. Por el Lema 4.1.9 tenemos que $X$ es débilmente $\omega_{1}$-Hausdorff por colecciones y aplicamos el Corolario 4.1.8.

Definición 4.1.11. Un espacio topológico $X$ tiene la condición de la cadena numerable (ccc) si cada familia ajena dos a dos de subconjuntos abiertos no vacíos en $X$ es numerable.

Lema 4.1.12. Sea $X$ un espacio topológico. Si $X$ tiene la ccc, entonces $X$ es tenuemente Lindelöf.

Demostración. Sea $\mathcal{U}$ una familia de tamaño $\omega_{1}$ localmente finita de subconjuntos abiertos no vacíos de $X$. Por el Lema 1.2.6 existe una familia $\mathcal{V}$ de tamaño $\omega_{1}$ ajena dos a dos de subconjuntos abiertos no vacíos en $X$. Se sigue que $X$ no tiene la ccc.

Definición 4.1.13. Un espacio topológico $X$ tiene la condición de la cadena discreta numerable (dccc) si cada familia discreta de subconjuntos abiertos no vacíos en $X$ es numerable.

Corolario 4.1.14. Cada espacio topológico $X$ que tiene la ccc tiene la decc.

Lema 4.1.15. Sea $X$ un espacio topológico regular. Entonces $X$ es tenuemente Lindelöf si y sólo si $X$ tiene la decc.

Demostración. $(\Rightarrow)$ Inmediato.

$(\Leftarrow)$ Sea $\mathcal{U}=\left\{U_{\alpha}: \alpha<\omega_{1}\right\}$ una familia localmente finita de subconjuntos abiertos no vacíos. Por el Lema 1.2.6 existe una familia localmente finita ajena dos a dos $\mathcal{V}=\left\{V_{\alpha}: \alpha<\omega_{1}\right\}$. Como $X$ es un espacio regular, para cada $\alpha<\omega_{1}$ existe $W_{\alpha} \in \tau(X)$ tal que $W_{\alpha} \subset \operatorname{cl}\left(W_{\alpha}\right) \subset V_{\alpha}$. Así la familia $\mathcal{W}=\left\{W_{\alpha}: \alpha<\omega_{1}\right\}$ es una familia discreta.

Lema 4.1.16. En el espacio $\mathbb{R}$ existe un subconjunto denso no numerable que se puede escribir como una unión no numerable de subconjuntos numerables ajenos dos a dos y densos en $\mathbb{R}$.

Demostración. Sea $D_{0}=\mathbb{Q}$, donde $\mathbb{Q}$ es el conjunto de los números racionales. Al haber escogido $D_{\beta}$ para cada $\beta<\alpha<\omega_{1}$, tal que $D_{\beta} \subset \mathbb{R} \backslash \bigcup\left\{D_{\gamma}: \gamma<\beta\right\}$ con $D_{\beta}$ numerable y denso en $\mathbb{R}$; se sigue que $\bigcup\left\{D_{\beta}: \beta<\alpha\right\}$ es numerable y por tanto, $\mathbb{R} \backslash \bigcup\left\{D_{\beta}: \beta<\alpha\right\}$ es denso en $\mathbb{R}$. Puesto que $\mathbb{R}$ es hereditariamente separable, existe un subconjunto numerable $D_{\alpha} \subseteq \mathbb{R} \backslash \bigcup\left\{D_{\beta}: \beta<\alpha\right\}$, el cual es denso en $\mathbb{R} \backslash \bigcup\left\{D_{\beta}: \beta<\alpha\right\}$ y por tanto denso en $\mathbb{R}$. De esta manera tenemos que $X=\bigcup\left\{D_{\beta}: \beta<\omega_{1}\right\} \subset \mathbb{R}$ es la unión de $\omega_{1}$ subconjuntos de $\mathbb{R}$, los cuales son numerables, ajenos dos a dos y densos en $\mathbb{R}$. 
Ejemplo 4.1.17. Existe un espacio topológico de Hausdorff que tiene la dccc, pero no es tenuemente Lindelöf.

Demostración. Sea $\left(\mathbb{R}, \tau_{e}\right)$ el espacio topológico de los números reales con la topología usual. Consideramos el subconjunto $X$ de $\mathbb{R}$ construido en el Lema 4.1.16 y lo escribimos como la siguiente unión ajena

$$
X=\left(\bigcup\left\{Y_{\alpha}: \alpha<\omega_{1}\right\}\right) \cup\left(\bigcup\left\{Z_{\alpha \beta}: \alpha, \beta<\omega_{1}\right\}\right)
$$

donde cada $Y_{\alpha}$ y cada $Z_{\alpha \beta}$ son densos numerables en $\left(\mathbb{R}, \tau_{e}\right)$. Consideremos la siguiente topología $\tau$ en $X$ generada por la siguiente base $\mathcal{W}$ :

1) Para cada $y \in Y_{\alpha}, V_{U_{y}, \alpha}=U_{y} \cap Y_{\alpha} \in \mathcal{W}$ con $U_{y} \in \tau_{e}(y, \mathbb{R})$.

2) Para cada $z \in Z_{\alpha \beta},\{z\} \cup\left(U_{z} \cap\left(Y_{\alpha} \cup Y_{\beta}\right)\right) \in \mathcal{W}$ con $U_{z} \in \tau_{e}(z, \mathbb{R})$.

La topología $\tau$ es más fina que la topología $\tau_{e}$, así $(X, \tau)$ es un espacio de Hausdorff. Para mostrar que $(X, \tau)$ no es tenuemente Lindelöf basta observar que $\left\{Y_{\alpha}: \alpha<\omega_{1}\right\}$ es una familia localmente finita de subconjuntos abiertos no vacíos.

Ahora mostremos que $(X, \tau)$ es un espacio topológico con la dccc. Sea $\mathcal{A}$ una familia no numerable de subconjuntos abiertos no vacíos en $(X, \tau)$. Sin pérdida de generalidad podemos asumir que $\mathcal{A}$ consiste de subconjuntos de la forma $V_{U, \alpha}$, donde $U \in \mathcal{B}$ y $\mathcal{B}$ es una base numerable de $\tau_{e}$. Como $\mathcal{B}$ es una base numerable y $\mathcal{A}$ es una familia no numerable, existen $\alpha_{1}, \alpha_{2}<\omega_{1}$ y $U^{\prime} \in \mathcal{B}$ tales que $V_{U^{\prime}, \alpha_{1}}, V_{U^{\prime}, \alpha_{2}} \in \mathcal{A}$. Como $Z_{\alpha_{1} \alpha_{2}}$ es un subconjunto denso en $\left(\mathbb{R}, \tau_{e}\right)$, existe $z \in Z_{\alpha_{1} \alpha_{2}} \cap U^{\prime}$. Entonces cada vecindad de $z$ en la topología $\tau$ intersecta al menos a dos elementos de la familia $\mathcal{A}$ que son $V_{U^{\prime}, \alpha_{1}}$ y $V_{U^{\prime}, \alpha_{2}}$. Así $\mathcal{A}$ no es una familia discreta, por lo tanto $(X, \tau)$ tiene la dccc.

Teorema 4.1.18. Si $X$ es un $P$-espacio normal, entonces las siguientes condiciones son equivalentes:

a) $X$ es estrella numerable;

b) $X$ estrella Lindelöf;

c) $X$ es tenuemente Lindelöf;

d) $X$ tiene la decc;

e) $\operatorname{ext}(X)=\omega$

Demostración. (e) $\Rightarrow$ (a) Es el Corolario 2.1.8; 
(a) $\Rightarrow$ (b) Es evidente;

(b) $\Rightarrow$ (c) Es el Teorema 3.1.8;

(c) $\Rightarrow$ (d) Es el Lema 4.1.15;

(d) $\Rightarrow$ (e) Supongamos que cada familia discreta de subconjuntos abiertos no vacíos de $X$ es numerable y que existe un conjunto cerrado y discreto $D$ en $X$ tal que $|D|=\omega_{1}$. Por el Lema 4.1.9 existe una expansión abierta ajena $\left\{U_{d}: d \in D\right\}$ para el subconjunto $D$. Ya que $X$ es un espacio normal, podemos encontrar un subconjunto abierto $G$ en $X$ tal que $D \subseteq G \subseteq \operatorname{cl}(G) \subseteq \bigcup\left\{U_{d}: d \in D\right\}$. Para cada $\alpha<\omega_{1}$ sea $V_{d}=G \cap U_{d}$, entonces la familia $\left\{V_{d}: d \in D\right\}$ es una expansión abierta y discreta para $D$.

Definición 4.1.19. Sean $(X, \tau)$ un espacio topológico $T_{1}$ y $F \subseteq X$. Denotaremos

$$
\psi(F, X)=\min \{\kappa: F \text { es la interseccion de } \kappa \text { subconjuntos abiertos }\} .
$$

Si $x \in X$ escribiremos $\psi(x, X)$ en lugar de $\psi(\{x\}, X)$.

Proposición 4.1.20. Sea $X$ un $P$-espacio de Tychonoff con la decc y $\psi(x, X) \leq \omega_{1}$ para algún $x \in X$. Entonces $\chi(x, X) \leq \omega_{1}$.

Demostración. Por el Lema 3.1.27 $X$ es un espacio 0-dimensional, ahora fijemos una $\omega_{1}$-sucesión decreciente $\mathcal{U}=\left\{U_{\alpha}: \alpha \in \omega_{1}\right\}$ de subconjuntos abiertos y cerrados de $X$ tales que $\{x\}=\bigcap \mathcal{U}$. Para ver que $\mathcal{U}$ es una base local en $x$, fijamos cualquier conjunto abierto y cerrado $U \in \tau(x, X)$ y observemos que en el espacio $Y=X \backslash U$, cada familia discreta de subconjuntos abiertos no vacíos es numerable porque $Y$ es abierto y cerrado en $X$.

Sea $V_{\alpha}=Y \backslash U_{\alpha}$ para cada $\alpha \in \omega_{1}$; entonces la familia $\mathcal{V}=\left\{V_{\alpha}: \alpha \in \omega_{1}\right\}$ es una cubierta por subconjuntos abiertos y cerrados de $Y$. Como $Y$ es un $P$-espacio, el conjunto $W_{\alpha}=V_{\alpha} \backslash \bigcup\left\{V_{\beta}: \beta<\alpha\right\}$ es un conjunto abierto y cerrado en $Y$ para $\alpha<\omega_{1}$ y por lo tanto tenemos que $\mathcal{W}=\left\{W_{\alpha}: \alpha \in \omega_{1}\right\}$ es un refinamiento de abiertos y cerrados de $\mathcal{V}$.

Este refinamiento es una familia discreta de subconjuntos de $Y$ así que sólo una cantidad numerable de elementos de $\mathcal{W}$ son no vacíos. Ya que la familia $\mathcal{V}$ es decreciente, tenemos que $Y \backslash U_{\alpha}=V_{\alpha}=Y$, es decir, $U_{\alpha} \subset U$ para algún $\alpha \in \omega_{1}$.

Proposición 4.1.21. Sea $X$ un $P$-espacio de Tychonoff con la decc y $l(X) \leq \omega_{1}$. Entonces $X$ es un espacio de Lindelöf.

Demostración. Sea $\mathcal{U}$ una cubierta abierta de $X$. Podemos asumir sin pérdida de generalidad que $|\mathcal{U}| \leq \omega_{1}$, y como $X$ es 0 -dimensional también podemos suponer que 
los elementos de $\mathcal{U}$ son abiertos y cerrados. Sean $\left\{U_{\alpha}: \alpha<\omega_{1}\right\}$ una enumeración de la familia $\mathcal{U}$ y $V_{\alpha}=U_{\alpha} \backslash \bigcup\left\{U_{\beta}: \beta<\alpha\right\}$ para cada $\alpha<\omega_{1}$. Es claro que $\mathcal{V}=\left\{V_{\alpha}: \alpha<\omega_{1}\right\}$ es una partición de $X$ que consta de subconjuntos abiertos y cerrados, así la familia $\mathcal{V}$ es discreta y por lo tanto la colección $\mathcal{W}=\{V \in \mathcal{V}: V \neq \emptyset\}$ es un refinamiento abierto numerable de la cubierta $\mathcal{U}$. Se sigue que $X$ es un espacio de Lindelöf.

Teorema 4.1.22. Si $\kappa$ es un cardinal infinito tal que $\kappa^{\omega}=\kappa$ y $X$ es un $P$-espacio de Tychonoff estrella Lindelöf tal que $w(X) \leq \kappa$, entonces $X$ no tiene ningún subespacio cerrado y discreto de cardinalidad $\kappa$; en particular, si $\kappa$ es un cardinal sucesor, entonces $e(X)<\kappa$.

Demostración. Supongamos que $D$ es un subconjunto cerrado y discreto de $X$ de cardinalidad $\kappa$. Podemos encontrar una base $\mathcal{B}$ de tamaño $\kappa$ para $X$ que consta de subconjuntos abiertos y cerrados tal que $|U \cap D| \leq 1$ para cada $U \in \mathcal{B}$.

Sea $\left\{\mathcal{V}_{\alpha}: \alpha<\kappa\right\}$ una enumeración de todas las subfamilias numerables de $\mathcal{B}$. Como $X$ es $P$-espacio tenemos que $\bigcup \mathcal{V}_{0}$ es un subconjunto cerrado de $X$ el cual contiene a lo más una cantidad numerable de elementos de $D$, así podemos elegir $d_{0} \in D \backslash \bigcup \mathcal{V}_{0}$.

Procediendo recursivamente, al haber elegido $d_{\beta}$ para cada $\beta<\alpha<\kappa$ tal que si $\beta \neq \beta^{\prime}$, entonces $d_{\beta} \neq d_{\beta^{\prime}}$ y $d_{\beta} \in D \backslash \bigcup \mathcal{V}_{\beta}$. Como el subconjunto $P=\left\{d_{\beta}: \beta<\alpha\right\} \cup\left(\bigcup \mathcal{V}_{\alpha}\right)$ contiene a lo más $|\alpha| \cdot \omega<\kappa$ elementos de $D$, podemos elegir $d_{\alpha} \in D \backslash P$. Sea $D^{\prime}=\left\{d_{\alpha}: \alpha<\kappa\right\} \subseteq D$. Veamos que $D^{\prime}$ es un subconjunto cerrado, sea $x \in X \backslash D^{\prime}$, si $x \in D \backslash D^{\prime}$, entonces existe $U \in \tau(x, X)$ tal que $U \cap D=\{x\}$; si $x \in X \backslash D$, como $D$ es cerrado existe $V \in \tau(x, X)$ ajeno a $D$ y por lo tanto a $D^{\prime}$, se sigue que $D^{\prime}$ es cerrado.

Fijamos $W_{\alpha} \in \tau\left(d_{\alpha}, X\right)$, tal que $W_{\alpha} \cap D^{\prime}=\left\{d_{\alpha}\right\}$ y $W_{\alpha} \cap\left(\bigcup \mathcal{V}_{\alpha}\right)=\emptyset$ para cada $\alpha<\kappa$. La familia $\mathcal{U}=\left\{X \backslash D^{\prime}\right\} \cup\left\{W_{\alpha}: \alpha<\kappa\right\}$ es una cubierta abierta de $X$. Por lo tanto existe un subespacio de Lindelöf $L \subseteq X$ tal que $S t(L, \mathcal{U})=X$. Como la familia $\mathcal{B}$ cubre a $L$, existe $\alpha<\kappa$ tal que $L \subseteq \bigcup \mathcal{V}_{\alpha}$. Como $W_{\alpha} \cap\left(\bigcup \mathcal{V}_{\alpha}\right)=\emptyset$, entonces $W_{\alpha} \cap L=\emptyset$; por lo que $d_{\alpha} \notin S t(L, \mathcal{U})$.

\subsection{Estrella $\mathrm{P}$ en espacios de Moore.}

En esta sección mostraremos que las propiedades estrella Lindelöf y estrella numerable que fueron estudiadas en el Capítulo 3 son equivalentes a la propiedad de separabilidad en una clase particular de espacios topológicos.

Definición 4.2.1. Decimos que un espacio topológico $X$ tiene un desarrollo si existe una sucesión de cubiertas abiertas $\left\{\mathcal{U}_{n}: n \in \omega\right\}$ de $X$ tal que para cada $x \in X$, la sucesión $\left\{S t\left(x, \mathcal{U}_{n}\right): n \in \omega\right\}$ es una base local para $x$. Un espacio es de Moore si es regular y tiene un desarrollo. 
El siguiente teorema es una generalización de un resultado de Ikenaga que puede encontrarse en [9], donde se probó que cada espacio de Moore estrella numerable es separable.

Teorema 4.2.2. Si $X$ es un espacio de Moore, entonces los siguientes enunciados son equivalentes:

(a) $X$ es un espacio separable;

(b) $X$ es estrella numerable;

(c) $X$ es estrella Lindelöf.

Demostración. (a) $\Rightarrow$ (b) $\Rightarrow$ (c) Inmediato.

(c) $\Rightarrow$ (a) Supongamos que $X$ es un espacio de Moore estrella Lindelöf; podemos encontrar un desarrollo $\left\{\mathcal{U}_{n}: n \in \omega\right\}$ para $X$. Sea $L_{n}$ un núcleo de Lindelöf de $\mathcal{U}_{n}$ para cada $n \in \omega$. Como $L_{n}$ es un espacio de Lindelöf y un espacio de Moore, por el Teorema 5.4.1 (Teorema de Metrización de Bing) de [6] se sigue que $L_{n}$ es metrizable para cada $n \in \omega$, por lo que existe un subespacio denso numerable $D_{n}$ de $L_{n}$ para cada $n \in \omega$.

Sean $D=\bigcup\left\{D_{n}: n \in \omega\right\}, x \in X$ y $U \in \tau(x, X)$. Existe $m \in \omega$ tal que $S t\left(x, \mathcal{U}_{m}\right) \subseteq U$. Como el subespacio $L_{m}$ es un núcleo de $\mathcal{U}_{m}$, podemos encontrar $z \in L_{m}$ y $V \in \mathcal{U}_{m}$ tal que $\{x, z\} \subset V$. Así tenemos $V \subseteq S t\left(x, \mathcal{U}_{m}\right) \subseteq U$; además como $V \cap L_{m} \neq \emptyset$ y $V \cap D_{m} \neq \emptyset$, esto demuestra que $U \cap D \neq \emptyset$ y por lo tanto $D$ es un subconjunto denso en $X$. 


\section{Capítulo 5}

\section{Estrellas débiles.}

\subsection{Estrella débil y casi estrella.}

En esta sección estudiaremos dos propiedades que surgen al debilitar la propiedad estrella $P$. Mostraremos para qué propiedades topológicas y bajo qué condiciones son equivalentes. El nombre de estas propiedades son débilmente estrella $P$ y casi estrella $P$ y fueron estudiadas en [16] cuando $P=$ finito y cuando $P=$ numerable, bajo los nombres de "1 - cl-starcompact" y "1 - cl-star-Lindelöf" respectivamente. El primero de estos términos es atribuido a Ikenaga [9]. Más tarde diversos autores como Yan-Kui Song y Wei-Feng Xuan en [22] estudiaron estas propiedades.

Definición 5.1.1. Un espacio topológico $X$ se llama débilmente estrella $P$ (casi estrella $P$, respectivamente) si para cada cubierta abierta $\mathcal{U}$ de $X$ existe un subespacio $A \subset X$ con la propiedad $P$ tal que $\operatorname{cl}(S t(A, \mathcal{U}))=X,(\bigcup\{\operatorname{cl}(S t(x, \mathcal{U})): x \in A\}=X$, respectivamente). El conjunto $A$ es llamado un núcleo débil (casi núcleo, respectivamente) de la cubierta $\mathcal{U}$.

Lema 5.1.2. Sea $P$ una propiedad topológica. Si $X$ es un espacio casi estrella $P$, entonces $X$ es débilmente estrella $P$.

Demostración. Sea $\mathcal{U}$ una cubierta abierta de $X$, entonces existe un conjunto $A \subset X$ con la propiedad $P$ tal que $\bigcup_{x \in A} \operatorname{cl}(S t(x, \mathcal{U}))=X$. El resultado es consecuencia de:

$$
\bigcup_{x \in A} \operatorname{cl}(S t(x, \mathcal{U})) \subseteq \operatorname{cl}(S t(A, \mathcal{U}))
$$

Proposición 5.1.3. Un espacio topológico $X$ con un subespacio denso estrella $P$ es débilmente estrella $P$. 
Demostración. Sea $D$ un espacio denso estrella $P$ en $X$ y sea $\mathcal{U}$ una cubierta abierta de $X$. Como $D$ es estrella $P$ existe $A \subseteq D$ con la propiedad $P$ tal que $D \subseteq S t\left(A, \mathcal{U}_{D}\right)$ (donde $\mathcal{U}_{D}=\{U \cap D: U \in \mathcal{U}\}$ ), así $X=\operatorname{cl}(D)=\operatorname{cl}(S t(A, \mathcal{U})$ ).

Corolario 5.1.4. Un espacio $X$ con un subespacio denso estrella numerable es débilmente estrella numerable.

Proposición 5.1.5. Si $X$ es un espacio casi estrella numerable y $A \subseteq X$ es un subespacio cerrado y abierto, entonces $A$ es casi estrella numerable.

Demostración. Sea $\mathcal{U}$ una cubierta abierta de $A$. Entonces $\mathcal{V}=\mathcal{U} \cup\{X \backslash A\}$ es una cubierta abierta de $X$. Ya que $X$ es casi estrella numerable podemos encontrar un subconjunto numerable $N$ en $X$ tal que $\bigcup\{\operatorname{cl}(S t(x, \mathcal{V})): x \in N\}=X$. Entonces $\bigcup\{\operatorname{cl}(S t(x, \mathcal{U})): x \in N \cap A\}=A$.

La siguiente definición aparece en [16].

Definición 5.1.6. Un espacio $X$ tiene la propiedad $D C\left(\omega_{1}\right)$ si tiene un subespacio $D$ denso tal que cada subconjunto no numerable de $D$ tiene un punto de acumulación en $X$.

Teorema 5.1.7. Cada espacio $X$ con la propiedad $D C\left(\omega_{1}\right)$ es débilmente estrella numerable.

Demostración. Sean $\mathcal{U}$ una cubierta abierta de $X$ y $D$ el subconjunto denso testigo de que $X$ tiene la propiedad $D C\left(\omega_{1}\right)$. Elegimos $x_{0} \in D ; \operatorname{sicl}\left(S t\left(x_{0}, \mathcal{U}\right)\right) \supseteq D$, entonces ya acabamos; de otra forma elegimos $x_{1} \in D \backslash \operatorname{cl}\left(S t\left(x_{0}, \mathcal{U}\right)\right)$. Al haber elegido puntos $x_{\alpha}$ para cada $\alpha<\beta$ de tal manera que $x_{\alpha} \in D \backslash \operatorname{cl}\left(S t\left(A_{\alpha}, \mathcal{U}\right)\right)$, donde $A_{\alpha}=\left\{x_{\gamma}: \gamma<\alpha\right\}$, elegimos $x_{\beta} \in D \backslash \operatorname{cl}\left(S t\left(A_{\beta}, \mathcal{U}\right)\right)$, donde $A_{\beta}=\left\{x_{\gamma}: \gamma<\beta\right\} ; \operatorname{sicl} \operatorname{cl}\left(S t\left(A_{\beta}, \mathcal{U}\right)\right) \supseteq D$ se termina la construcción. Se demostrará que esto sí ocurre para algún $\beta<\omega_{1}$.

Supongamos que esto no ocurre; sea $A=\left\{x_{\alpha}: \alpha<\omega_{1}\right\}$; $A$ es discreto pues $\left\{x_{\delta}\right\}=$ $\left(X \backslash \operatorname{cl}\left(S t\left(A_{\delta}, \mathcal{U}\right)\right)\right) \cap S t\left(x_{\delta}, \mathcal{U}\right) \cap A$ para cada $\alpha<\omega_{1}$. Como $X$ tiene la propiedad $D C\left(\omega_{1}\right)$ tenemos que $A$ tiene un punto de acumulación; sea $p \in X$ tal punto, por lo tanto existe $U \in \mathcal{U}$ tal que $p \in U$. Como $p$ es un punto de acumulación de $A$, existe un $\beta \in \omega_{1}$ mínimo tal que $x_{\beta} \in U$ y por la construcción, para todo $\gamma \neq \beta, x_{\gamma} \notin U$ lo que contradice que $p$ es punto de acumulación de $A$. Así que para algún ordinal $\delta \in \omega_{1}$, se tiene que $\operatorname{cl}\left(S t\left(A_{\delta}, \mathcal{U}\right)\right) \supseteq D$. Por lo tanto $X$ es débilmente estrella numerable.

Corolario 5.1.8. Si un espacio $X$ tiene un subespacio denso con extensión numerable, entonces $X$ es débilmente estrella numerable. 
Demostración. Sea $S$ un subespacio denso de $X$ con extensión numerable, entonces cada subconjunto no numerable de $S$ tiene un punto de acumulación en $S$.

Corolario 5.1.9. Cada espacio con un subespacio denso $\sigma$-numerablemente compacto, es débilmente estrella numerable.

Demostración. Sea $S$ el subespacio denso $\sigma$-numerablemente compacto, entonces $S=$ $\bigcup\left\{S_{n}: n \in \omega\right\}$ con cada $S_{n}$ numerablemente compacto. Sea $A$ un conjunto infinito no numerable en $S$, entonces existe $m \in \omega$ tal que $A \cap S_{m}$ es no numerable, por lo tanto tiene punto de acumulación $p \in S_{m} \subseteq S$.

Corolario 5.1.10. Cada espacio $X$ con un subespacio denso de Lindelöf, es débilmente estrella numerable.

Definición 5.1.11. Un espacio $X$ se llama débilmente de Lindelöf si cada cubierta abierta de $X$ tiene una subfamilia numerable cuya unión es densa en $X$.

Lema 5.1.12. Un espacio $X$ débilmente de Lindelöf es débilmente estrella numerable.

Demostración. Sea $\mathcal{U}$ una cubierta abierta de $X$. Entonces existe $\left\{V_{n}: n \in \omega\right\} \subseteq \mathcal{U}$ tal que $\operatorname{cl}\left(\bigcup\left\{V_{n}: n \in \omega\right\}\right)=X$. Para cada $n \in \omega$ elegimos $x_{n} \in V_{n}$. Sea $F=\left\{x_{n}: n \in\right.$ $\omega\}$, entonces $S t(F, \mathcal{U})=\bigcup\left\{V_{n}: n \in \omega\right\}$. Por lo tanto $\operatorname{cl}(S t(F, \mathcal{U}))=X$.

Lema 5.1.13. Un espacio $X$ con celularidad numerable es débilmente de Lindelöf.

Demostración. Supongamos que $X$ no es débilmente de Lindelöf y sea $\mathcal{U}$ una cubierta abierta de $X$ tal que ninguna subfamilia numerable tiene unión densa en $X$. Sean $U_{0} \in \mathcal{U}$ con $\operatorname{cl}\left(U_{0}\right) \neq X$ y $V_{0}=U_{0}$; al haber elegido $U_{\beta} \in \mathcal{U}$ para cada $\beta<\alpha<\omega_{1}$ tal que $\operatorname{cl}\left(\bigcup\left\{U_{\beta}: \beta<\alpha\right\}\right) \neq X$. Podemos encontrar $U_{\alpha} \in \mathcal{U}$ tal que $U_{\alpha} \backslash \operatorname{cl}\left(\bigcup\left\{U_{\beta}: \beta<\right.\right.$ $\alpha\}) \neq \emptyset$, hacemos $V_{\alpha}=U_{\alpha} \backslash \operatorname{cl}\left(\bigcup\left\{U_{\beta}: \beta<\alpha\right\}\right)$; así la familia $\left\{V_{\alpha}: \alpha<\omega_{1}\right\}$ es una familia celular en $X$.

Teorema 5.1.14. Un espacio $X$ con celularidad numerable es débilmente estrella numerable.

Demostración. Es inmediato de los dos lemas anteriores.

Ejemplo 5.1.15. Existe un espacio $X$ con un subespacio denso y $\sigma$-compacto que no es casi estrella numerable.

Demostración. Denotemos por $\lambda\left(\omega_{1}\right)$ el conjunto de ordinales límites en $\omega_{1}+1$. Sea $X=\left(\left(\omega_{1}+1\right) \times(\omega+1)\right) \backslash\left\{(\alpha, \omega): \alpha \in \lambda\left(\omega_{1}\right)\right\}$ como subespacio del espacio producto $\left(\omega_{1}+1\right) \times(\omega+1)$. El subespacio $Y=\left(\omega_{1}+1\right) \times(\omega)$ es $\sigma$-compacto y denso en $X$. Para 
ver que $X$ no es casi estrella numerable, sea $\mathcal{U}$ la cubierta abierta de $X$ definida de la siguiente manera:

$$
\mathcal{U}=\left\{\left(\omega_{1}+1\right) \times\{n\}: n \in \omega\right\} \cup\left\{\{\beta\} \times(\omega+1): \beta \in \omega_{1} \backslash \lambda\left(\omega_{1}\right)\right\} .
$$

Si $A$ es un subconjunto numerable de $\omega_{1} \times \omega$, entonces podemos encontrar $\alpha \in \omega_{1}$ tal que $A \subseteq \alpha \times \omega$ y así si $\gamma \geq \alpha$ tenemos que $(\gamma, \omega) \notin \mathrm{cl}(\operatorname{St}(x, \mathcal{U}))$ para cada $x \in A$.

Teorema 5.1.16. Si $X$ es un $P$-espacio, entonces las siguientes condiciones son equivalentes:

a) $X$ es débilmente estrella numerable;

b) $X$ es casi estrella numerable;

además, las dos condiciones anteriores son consecuencias del siguiente enunciado:

c) $X$ tiene un subespacio denso con extensión numerable;

si además, $X$ es un espacio normal, entonces las tres condiciones anteriores son equivalentes a:

d) $X$ es estrella numerable.

Demostración. (b) $\Rightarrow$ (a) Es consecuencia del Lema 5.1.2.

(a) $\Rightarrow$ (b) Se sigue del hecho de que si $\left\{C_{n}: n \in \omega\right\}$ es una familia numerable de conjuntos en un $P$-espacio, entonces $\bigcup\left\{\operatorname{cl}\left(C_{n}\right): n \in \omega\right\}=\operatorname{cl}\left(\bigcup\left\{C_{n}: n \in \omega\right\}\right)$.

(c) $\Rightarrow$ (a) Es consecuencia del Corolario 5.1.8.

Ahora supongamos que $X$ es un espacio normal:

(a) $\Rightarrow$ (c) Supongamos que $X$ no tiene subespacio denso con extensión numerable. En particular $e(X)>\omega$ y así podemos encontrar un subconjunto cerrado y discreto $D=\left\{d_{\alpha}: \alpha \in \omega_{1}\right\} \subseteq X$ de cardinalidad $\omega_{1}$. Como $X$ es un $P$-espacio, por el Lema 4.1.9, podemos encontrar una expansión ajena de conjuntos abiertos $\mathcal{U}=\left\{U_{\alpha}: \alpha \in \omega_{1}\right\}$ de $D$, donde $d_{\alpha} \in U_{\alpha}$. Como $X$ es normal podemos asumir que $\mathcal{U}$ es discreta. Para cada $\alpha \in \omega_{1}$, podemos encontrar una vecindad cerrada $V_{\alpha}$ de $d_{\alpha}$ tal que $V_{\alpha} \subseteq U_{\alpha}$ y la cubierta $\mathcal{F}=\mathcal{U} \cup\left\{X \backslash \bigcup\left\{V_{\alpha}: \alpha \in \omega_{1}\right\}\right\}$ es testigo de que $X$ no es débilmente estrella numerable.

(b) $\Rightarrow$ (d) Notemos que si $X$ no es estrella numerable, por el Corolario 2.1.8 $X$ tiene extensión no numerable y por el argumento del párrafo anterior se tiene que $X$ no es 
débilmente estrella numerable.

(d) $\Rightarrow$ (a) Es trivial.

No obstante, en general las propiedades débilmente estrella numerable y casi estrella numerable son distintas. El espacio estudiado en el Ejemplo 5.1.15 es débilmente estrella numerable por el Lema 5.1.9 pero no es casi estrella numerable. A continuación exhibimos otros ejemplos que distinguen estas propiedades.

Lema 5.1.17. Un espacio $X$ que tiene un subespacio denso numerablemente compacto es casi estrella finito (por lo tanto es débilmente estrella finito).

Demostración. Sea $D$ un subespacio denso numerablemente compacto de $X$ y sea $\mathcal{U}$ una cubierta abierta de $X$. Como $D$ es un subconjunto denso numerablemente compacto de $X$, existe un subconjunto finito $F$ de $D$ tal que $D \subseteq \bigcup\{S t(x, \mathcal{U}): x \in F\}$. Notemos que las propiedades débilmente estrella finito y casi estrella finito son equivalentes. Por lo tanto, $X=\operatorname{cl}(D) \subseteq \operatorname{cl}(\bigcup\{S t(x, \mathcal{U}): x \in F\})$.

Ejemplo 5.1.18. Existe un espacio de Tychonoff $X$, el cual es casi estrella numerable pero no estrella numerable.

Demostración. Sea $D$ un espacio discreto de cardinalidad $\omega_{1}$, sea $X=\left(\beta D \times\left(\omega_{1}+\right.\right.$ $1)) \backslash\left((\beta D \backslash D) \times\left\{\omega_{1}\right\}\right)$ con la topología de subespacio del producto $\beta D \times\left(\omega_{1}+1\right)$. Como $\beta D \times \omega_{1}$ es un subespacio denso numerablemente compacto de $X$, entonces $X$ es casi estrella finita por el Lema 5.1.17. Ahora mostraremos que $X$ no es estrella numerable.

Como $|D|=\omega_{1}$, podemos enumerar a $D$ como $\left\{d_{\alpha}: \alpha \in \omega\right\}$. Para cada $\alpha \in \omega_{1}$, sea $U_{\alpha}=\left\{d_{\alpha}\right\} \times\left(\omega_{1}+1\right)$, entonces $U_{\alpha} \cap U_{\beta}=\emptyset$ si $\alpha \neq \beta$. Consideramos la cubierta abierta $\mathcal{U}=\left\{U_{\alpha}: \alpha \in \omega_{1}\right\} \cup\left\{\beta D \times \omega_{1}\right\}$ de $X$. Es suficiente mostrar que para cada subconjunto numerable $F$ de $X$ existe un punto $x \in X$ tal que $x \notin S t(F, \mathcal{U})$. Sea $F$ un conjunto numerable de $X$, entonces existe un $\gamma<\omega_{1}$ tal que $U_{\alpha} \cap F=\emptyset$ para cada $\alpha>\gamma$. Si elegimos $\beta>\gamma$, entonces $\left(d_{\beta}, \omega_{1}\right) \notin S t(F, \mathcal{U})$ ya que $U_{\beta}$ es en único elemento de $\mathcal{U}$ que contiene a $\left(d_{\beta}, \omega_{1}\right)$.

Ejemplo 5.1.19. Existe un espacio de Tychonoff, débilmente estrella numerable que no es casi estrella numerable.

Demostración. Sea $D$ un espacio discreto de cardinalidad $\omega_{1}$ y sea

$$
X=(\beta D \times(\omega+1)) \backslash((\beta D \backslash D) \times\{\omega\})
$$

como subespacio del producto $\beta D \times(\omega+1)$. Como $\beta D \times \omega$ es un subespacio denso $\sigma$ compacto de $X$, entonces $X$ es débilmente estrella numerable por el Lema 5.1.9, ahora 
mostraremos que $X$ no es casi estrella numerable.

Como $|D|=\omega_{1}$, podemos enumerar a $D$ como $\left\{d_{\alpha}: \alpha \in \omega_{1}\right\}$. Para cada $\alpha \in \omega_{1}$, sea $U_{\alpha}=\left\{d_{\alpha}\right\} \times(\omega+1)$, entonces $U_{\alpha} \cap U_{\beta}=\emptyset$ si $\alpha \neq \beta$ y para cada $n \in \omega$ sea $V_{n}=\beta D \times\{n\}$. Consideramos la cubierta abierta $\mathcal{U}=\left\{U_{\alpha}: \alpha \in \omega_{1}\right\} \cup\left\{V_{n}: n \in \omega\right\}$ de $X$. Sea $F$ un subconjunto numerable de $X$, notemos que $S t(x, \mathcal{U})=\operatorname{cl}(S t(x, \mathcal{U}))$, si $x=\left(d_{\alpha}, n\right)$ entonces $S t(x, \mathcal{U})=\left(\left\{d_{\alpha}\right\} \times(\omega+1)\right) \cup(\beta D \times\{n\})$, entonces $X \neq \bigcup\{\mathrm{cl}$ $(S t(x, \mathcal{U})): x \in F\}$.

Definición 5.1.20. Un espacio $X$ es débilmente regular si cada conjunto abierto contiene un dominio cerrado no vacío.

Teorema 5.1.21. Un espacio $X$ débilmente regular y débilmente estrella numerable es tenuemente Lindelöf.

Demostración. Supongamos que $X$ no es tenuemente Lindelöf. Entonces, por el Lema 1.2.6 existe una familia localmente finita no numerable de conjuntos abiertos no vacíos y mutuamente ajenos $\mathcal{U}=\left\{U_{\alpha}: \alpha \in \omega_{1}\right\}$. Como $X$ es débilmente regular para cada $\alpha \in \omega_{1}$ podemos encontrar un conjunto abierto no vacío $V_{\alpha}$ tal que cl $\left(V_{\alpha}\right) \subseteq U_{\alpha}$. Ahora consideremos la siguiente cubierta abierta $\mathcal{V}=\mathcal{U} \cup\left\{X \backslash \bigcup\left\{\mathrm{cl}\left(V_{\alpha}\right): \alpha \in \omega_{1}\right\}\right\}$. Por el Lema 1.2.8 el subconjunto $\bigcup\left\{c l\left(V_{\alpha}\right): \alpha \in \omega_{1}\right\}$ es cerrado.

Si $D \subseteq X$ es numerable, entonces como cada punto de $D$ está en a lo más dos elementos de $\mathcal{V}$, se sigue que existe algún $\beta \in \omega_{1}$ tal que $D \cap U_{\beta}=\emptyset$. Claramente, si $p \in V_{\beta}$, entonces $p \notin \operatorname{cl}(\bigcup\{S t(x, \mathcal{V}): x \in D\})$ y así $\operatorname{cl}(\bigcup\{S t(x, \mathcal{V}): x \in D\}) \neq X$.

Teorema 5.1.22. Si $X$ es un espacio pseudocompacto y casi estrella numerable, entonces cada espacio $Y$ tal que $X \subseteq Y \subseteq \beta X$ es casi estrella numerable.

Demostración. Supongamos que $\mathcal{U}$ es una cubierta abierta de $Y$; para cada $U \in \mathcal{U}$ existe un subconjunto abierto $V_{U}$ de $\beta X$ tal que $U=Y \cap V_{U}$. Entonces $\mathcal{V}=\left\{V_{U} \cap X\right.$ : $U \in \mathcal{U}\}$ es una cubierta abierta de $X$, por lo tanto existe un subconjunto numerable $C \subseteq X$ que cumple lo siguiente $\bigcup\left\{\operatorname{cl}_{X}(S t(x, \mathcal{V})): x \in C\right\}=X$. Además, $\operatorname{St}(x, \mathcal{V})=$ $X \cap\left(\cup\left\{V_{U}: x \in V_{U}\right\}\right)$ y así tenemos que $\operatorname{cl}_{\beta X}(S t(x, \mathcal{V}))=\operatorname{cl}_{\beta X}\left(\cup\left\{V_{U}: x \in V_{U}\right\}\right)$. Como $C$ es numerable se sigue que:

$$
F=\bigcup_{x \in C} \mathrm{cl}_{\beta X}\left(\bigcup\left\{V_{U}: x \in V_{U}\right\}\right)
$$

es un conjunto $F_{\sigma}$ en $\beta X$ que contiene a $X$. Como $X$ es pseudocompacto por el Teorema 1.2.26 se tiene que $F=\beta X$. Pero como $Y$ es denso en $\beta X$ se tiene que:

$$
\operatorname{cl}_{\beta X}\left(\bigcup\left\{V_{U}: x \in V_{U}\right\}\right)=\operatorname{cl}_{\beta X}\left(\bigcup\left\{V_{U} \cap Y: x \in V_{U}\right\}\right) .
$$


Además:

$$
\operatorname{cl}_{\beta X}\left(\bigcup\left\{V_{U} \cap Y: x \in V_{U}\right\}\right) \cap Y=\operatorname{cl}_{Y}\left(\bigcup\left\{V_{U} \cap Y: x \in V_{U}\right\}\right)=\operatorname{cl}_{Y}(S t(x, \mathcal{U})) .
$$

Por último tomamos la unión sobre $C$ y obtenemos:

$$
Y=\bigcup_{x \in C} \operatorname{cl}_{\beta X}\left(\bigcup\left\{V_{U}: x \in V_{U}\right\}\right) \cap Y=\bigcup_{x \in C} \operatorname{cl}_{Y}(S t(x, \mathcal{U}))
$$

Teorema 5.1.23. Si $X$ es un espacio débilmente estrella numerable, entonces cada subconjunto abierto y cerrado también es débilmente estrella numerable.

Demostración. Sea $A$ un conjunto abierto y cerrado de $X$ y $\mathcal{V}$ una cubierta abierta de $A$. Entonces la cubierta $\mathcal{U}=\mathcal{V} \cup\{X \backslash A\}$ es una cubierta abierta de $X$, por lo que existe un conjunto numerable $F \subseteq X$ tal que $\operatorname{cl}(S t(F, \mathcal{U}))=X$. Podemos ver que $A \cap F$ es un núcleo débil numerable para $\mathcal{V}$.

Teorema 5.1.24. Si $X$ es un espacio normal tenuemente Lindelöf, entonces $X$ es débilmente estrella numerable.

Demostración. Supongamos que existe una cubierta abierta $\mathcal{U}$ de $X$ tal que para cada conjunto numerable $F \subseteq X$ se tiene $\operatorname{cl}(S t(F, \mathcal{U})) \neq X$. Sea $x_{0} \in X$; podemos elegir $x_{1} \in X \backslash \operatorname{cl}\left(S t\left(\left\{x_{0}\right\}, \mathcal{U}\right)\right)$. Al haber elegido $\left\{x_{\alpha}: \alpha<\beta\right\}$ para algún $\beta<\omega_{1}$ podemos elegir un elemento $x_{\beta} \in X \backslash \operatorname{cl}\left(\operatorname{St}\left(\left\{x_{\alpha}: \alpha<\beta\right\}, \mathcal{U}\right)\right)$.

Para $\xi<\omega_{1}$, sea $V_{\xi}=S t\left(x_{\xi}, \mathcal{U}\right) \backslash \operatorname{cl}\left(S t\left(\left\{x_{\beta}: \beta<\xi\right\}, \mathcal{U}\right)\right)$. Entonces $V_{\xi}$ es un conjunto abierto, $x_{\xi} \in V_{\xi}$ y $V_{\xi} \cap V_{\gamma}=\emptyset$ si $\xi \neq \gamma$. Sea $G=\left\{x_{\xi}: \xi<\omega_{1}\right\}$. Veamos que $G$ es un subconjunto cerrado. Para ello sea $x \in X \backslash G$, si $x \in \bigcup\left\{V_{\xi}: \xi<\omega_{1}\right\}$, entonces existe $\delta<\omega_{1}$ tal que $x \in V_{\delta}$, entonces $V_{\delta} \backslash\left\{x_{\delta}\right\}$ es una vecindad de $x$ ajena de $G$, pues $G \cap V_{\delta}=\left\{x_{\delta}\right\}$. Si $x \in X \backslash \bigcup\left\{V_{\xi}: \xi<\omega_{1}\right\}$, como $X$ es normal y $X \backslash \bigcup\left\{V_{\xi}: \xi<\omega_{1}\right\}$ es un subconjunto cerrado, existen subconjuntos abiertos ajenos $O, U$ tales que $x \in O$ y $X \backslash \bigcup\left\{V_{\xi}: \xi<\omega_{1}\right\} \subset U$, así $O$ es una vecindad de $x$ ajena a $G$. Por lo tanto $G$ es un conjunto cerrado en $X$ y para cada $U \in \mathcal{U}$ se tiene que $\left|\left\{\alpha: x_{\alpha} \in U\right\}\right| \leq 1$. Como $X$ es normal existe un conjunto abierto $W$ de $X$ tal que $G \subseteq W \subseteq \operatorname{cl}(W) \subseteq \bigcup\left\{V_{\xi}: \xi<\omega_{1}\right\}$.

Para cada $\xi<\omega_{1}$, sea $W_{\xi}=W \cap V_{\xi}$. Entonces $\left\{W_{\xi}: \xi<\omega_{1}\right\}$ es una familia localmente finita no numerable de conjuntos abiertos no vacíos. De hecho, para cada $x \in X$ tenemos los siguientes casos:

1) Si $x \notin \operatorname{cl}(W)$, entonces $x \in X \backslash \operatorname{cl}(W)$ y $(X \backslash \operatorname{cl}(W)) \cap W_{\xi}=\emptyset$ para cada $\xi<\omega_{1}$.

2) Si $x \in \operatorname{cl}(W)$, entonces existe un $\xi<\omega_{1}$ tal que $x \in V_{\xi}$ y $V_{\xi} \cap V_{\gamma}=\emptyset$ si $\xi \neq \gamma$.

En consecuencia $X$ no es tenuemente de Lindelöf. 
Teorema 5.1.25. La imagen continua de un espacio débilmente estrella numerable es débilmente estrella numerable.

Demostración. Sean $X$ un espacio débilmente estrella numerable y $f: X \rightarrow Y$ una función continua y sobreyectiva. Si $\mathcal{U}$ es una cubierta abierta de $Y$, entonces la familia $\mathcal{V}=\left\{f^{-1}(U): U \in \mathcal{U}\right\}$ es una cubierta abierta de $X$ y como $X$ es débilmente estrella numerable existe un conjunto numerable $F \subseteq X$ tal que $\operatorname{cl}(\operatorname{St}(F, \mathcal{V}))=X$. Como $\operatorname{cl}(S t(F, \mathcal{V}))=X$, tenemos que $Y=f[X]=f[\operatorname{cl}(S t(F, \mathcal{V}))] \subseteq \operatorname{cl}(f[S t(F, \mathcal{V})]) \subseteq$ $\operatorname{cl}(S t(f[F], \mathcal{U}))$.

Teorema 5.1.26. Si $X$ es un espacio estrella numerable y $Y$ es un espacio separable, entonces $X \times Y$ es débilmente estrella numerable.

Demostración. Sea $D$ un subconjunto de $Y$ numerable y denso. Entonces $X \times D$ es un subconjunto denso y estrella numerable de $X \times Y$ y por el Corolario 5.1.4 se tiene el resultado.

Teorema 5.1.27. Si $X$ es un espacio de Hausdorff paracompacto y débilmente estrella numerable, entonces $X$ es de Lindelöf.

Demostración. Sea $\mathcal{U}$ una cubierta abierta de $X$. Como $X$ es regular, por el Lema 1.2.10, para cada $x \in X$ y cada $U \in \mathcal{U}$ tal que $x \in U$ se puede elegir un subconjunto abierto $V_{x}$ tal que $x \in V_{x} \subseteq \operatorname{cl}\left(V_{x}\right) \subseteq U$. Como $X$ es paracompacto existe un refinamiento localmente finito $\mathcal{V}$ de $\left\{V_{x}: x \in X\right\}$. Puesto que $X$ es débilmente estrella numerable, existe un conjunto numerable $F \subseteq X$ tal que $X=\operatorname{cl}(S t(F, \mathcal{V}))$. Sea $\mathcal{W}=\{V \in \mathcal{V}: V \cap F \neq \emptyset\}$ y observemos que $\mathcal{W}$ es numerable pues $\mathcal{V}$ es localmente finita y $F$ es numerable. Para cada $W \in \mathcal{W}$, elegimos $U_{W} \in \mathcal{U}$ tal que $\operatorname{cl}(W) \subseteq U_{W}$. Entonces $\left\{U_{W}: W \in \mathcal{W}\right\}$ es una subcubierta numerable de $X$, ya que $X=\operatorname{cl}(S t(F, \mathcal{V}))=\bigcup\{\operatorname{cl}(W): W \in \mathcal{W}\} \subseteq \bigcup\left\{U_{W}: W \in \mathcal{W}\right\} ;$ así $X$ es de Lindelöf.

Corolario 5.1.28. Si $X$ es un espacio de Hausdorff paracompacto, entonces las siguientes condiciones son equivalentes:

a) $X$ es de Lindelöf;

b) $X$ es estrella numerable;

c) $X$ es débilmente estrella numerable.

Demostración. (a) $\Rightarrow$ (b) Es la Proposición 3.1.2.

(b) $\Rightarrow$ (c) Obvio. 
(c) $\Rightarrow$ (a) Es el Teorema 5.1.27.

Una pregunta interesante que proponen Wei-Feng Xuan y Yan-Kui Song en [23] es:

Pregunta 5.1.29. ¿Existe un espacio normal casi estrella numerable que no es estrella numerable?

\subsection{Espacios débilmente estrella finita.}

En esta sección estudiaremos relaciones entre las propiedades tenuemente compacto y débilmente estrella finito. Observemos que las propiedades débilmente estrella finita y casi estrella finita son equivalentes; así que basta estudiar una de estas dos. Empezaremos con algunos resultados análogos a la sección anterior.

Definición 5.2.1. Un espacio $X$ tiene la propiedad $D C(\omega)$ si tiene un subespacio denso $D$ tal que cada subconjunto numerable infinito de $D$ tiene un punto de acumulación en $X$.

Teorema 5.2.2. Si $X$ es un espacio topológico con la propiedad $D C(\omega)$, entonces $X$ es débilmente estrella finita.

Demostración. Sean $\mathcal{U}$ una cubierta abierta de $X$ y $D$ el subconjunto denso testigo de que $X$ tiene la propiedad $D C(\omega)$. Elegimos $x_{0} \in D ; \operatorname{si} \operatorname{cl}\left(S t\left(x_{0}, \mathcal{U}\right)\right) \supseteq D$, entonces ya acabamos; de otra forma elegimos $x_{1} \in D \backslash \operatorname{cl}\left(S t\left(x_{0}, \mathcal{U}\right)\right)$. Al haber elegido puntos $x_{k}$ para cada $k \leq n$ de tal manera que $x_{k} \in D \backslash \operatorname{cl}\left(S t\left(A_{k}, \mathcal{U}\right)\right)$, donde $A_{k}=\left\{x_{m}: m<k\right\}$; si cl $\left(\operatorname{St}\left(A_{n}, \mathcal{U}\right)\right) \supseteq D$, se termina la construcción. Se demostrará que esto sí ocurre para algún $n<\omega$.

Supongamos que esto no ocurre. Sea $A=\left\{x_{n}: n<\omega\right\} ; A$ es discreto pues $\left\{x_{l}\right\}=$ $\left(X \backslash \operatorname{cl}\left(S t\left(A_{l}, \mathcal{U}\right)\right)\right) \cap S t\left(x_{l}, \mathcal{U}\right) \cap A$ para cada $l<\omega$. Como $X$ tiene la propiedad $D C(\omega)$ tenemos que $A$ tiene un punto de acumulación; sea $p \in X$ tal punto, por lo tanto existe $U \in \mathcal{U}$ tal que $p \in U$. Como $p$ es un punto de acumulación de $A$, existe un $m \in \omega$ mínimo tal que $x_{m} \in U$ y por la construcción, para todo $k \neq m, x_{k} \notin U$ lo que contradice que $p$ es punto de acumulación. Así para algún $n \in \omega$, se tiene que $\operatorname{cl}\left(S t\left(A_{n}, \mathcal{U}\right)\right) \supseteq D$, así concluimos que $X$ es débilmente estrella finita.

Teorema 5.2.3. Un espacio $X$ débilmente regular y débilmente estrella finita es tenuemente compacto.

Demostración. Si un espacio $X$ no es tenuemente compacto, entonces por el Lema 1.2.6 existe una familia infinita numerable localmente finita de conjuntos abiertos no vacíos y mutuamente ajenos $\mathcal{U}=\left\{U_{\alpha}: \alpha \in \omega\right\}$. Como $X$ es débilmente regular para 
cada $\alpha \in \omega$, podemos encontrar un conjunto abierto no vacío $V_{\alpha}$ tal que $\operatorname{cl}\left(V_{\alpha}\right) \subseteq U_{\alpha}$. Por Lema 1.2.8 sabemos que el conjunto $\bigcup\left\{\operatorname{cl}\left(V_{\alpha}\right): \alpha \in \omega\right\}$ es cerrado. Ahora consideremos la cubierta abierta $\mathcal{V}=\mathcal{U} \cup\left\{X \backslash \bigcup\left\{\operatorname{cl}\left(V_{\alpha}\right): \alpha \in \omega\right\}\right\}$.

Si $D \subseteq X$ es un conjunto finito de $X$, entonces como cada punto de $D$ está en a lo más dos elementos de $\mathcal{V}$, se sigue que existe algún $\beta \in \omega$ tal que $D \cap U_{\beta}=\emptyset$. Claramente, si $p \in V_{\beta}$, entonces $p \notin \operatorname{cl}(\bigcup\{S t(x, \mathcal{U}): x \in D\})$ y así $\operatorname{cl}(\bigcup\{S t(x, \mathcal{U}): x \in D\}) \neq X$.

Teorema 5.2.4. Si $X$ es un espacio tenuemente compacto y estrella numerable, entonces $X$ es débilmente estrella finito.

Demostración. Sea $\mathcal{U}$ una cubierta abierta de $X$. Como $X$ es estrella numerable, existe un conjunto $A=\left\{x_{n}: n \in \omega\right\} \subseteq X$ tal que $S t(A, \mathcal{U})=X$. Para cada $n \in \omega$, sea $F_{n}=S t\left(x_{n}, \mathcal{U}\right)$. Entonces $\mathcal{F}=\left\{F_{n}: n \in \omega\right\}$ es una cubierta abierta numerable de $X$ pues $\bigcup \mathcal{F}=S t(A, \mathcal{U})=X$. Como $X$ es tenuemente compacto, por el inciso (4) del Lema 1.2.3 existe un subcolección finita $\mathcal{G}=\left\{F_{n_{1}}, \ldots, F_{n_{k}}\right\} \subset \mathcal{F}$ tal que $\operatorname{cl}(\bigcup \mathcal{G})=$ $\operatorname{cl}\left(\operatorname{St}\left(\left\{n_{1}, \ldots, n_{k}\right\}, \mathcal{U}\right)\right)=X$.

Corolario 5.2.5. Un espacio separable tenuemente compacto es débilmente estrella finito.

Demostración. Se sigue del hecho que cada espacio separable es estrella numerable.

\subsection{Espacios metacompactos y metaLindelöf.}

Definición 5.3.1. Una colección $\mathcal{F}$ de subconjuntos de un espacio $X$ se llama punto finita si cada $x \in X$ pertenece a lo más a un número finito de elementos de la colección $\mathcal{F}$.

Definición 5.3.2. Un espacio topológico $X$ es llamado metacompacto si cada cubierta abierta de $X$ tiene un refinamiento abierto punto finita.

Definición 5.3.3. Un espacio topológico $X$ es llamado metaLindelöf si cada cubierta abierta de $X$ tiene un refinamiento abierto punto numerable.

Definición 5.3.4. Un espacio topológico $X$ es $H$-cerrado si para cada cubierta abierta $\mathcal{U}$ de $X$ existe una subfamilia finita de $\mathcal{U}$ cuya unión es densa en $X$.

Lema 5.3.5. Si $X$ es un espacio topológico separable, entonces tiene la ccc.

Demostración. Sean $D$ un subespacio denso numerable en $X$ y $\mathcal{U}$ una familia de subconjuntos abiertos no vacíos y mutuamente ajenos. Para cada $U \in \mathcal{U}$ existe $x_{U} \in$ $D \cap U$; si $U, V \in \mathcal{U}$ y $U \neq V$, entonces $U \cap V=\emptyset$, así $x_{U} \neq x_{V}$. Se sigue que la función $f: \mathcal{U} \rightarrow D$ es inyectiva, por lo que $|\mathcal{U}| \leq|D|$, es decir, $\mathcal{U}$ es numerable. 
Teorema 5.3.6. Un espacio de Hausdorff metaLindelöf y débilmente estrella numerable es tenuemente Lindelöf.

Demostración. Sea $X$ un espacio metaLindelöf Hausdorff y débilmente estrella numerable y sea $\mathcal{U}$ una familia localmente finita de conjuntos abiertos de $X$. Entonces para cada $x \in X$ existe un conjunto abierto $V_{x}$ que contiene a $x$ y que intersecta a lo más a un número finito de elementos de $\mathcal{U}$. Así la familia $\mathcal{V}=\left\{V_{x}: x \in X\right\}$ es una cubierta abierta de $X$. Como $X$ es metaLindelöf, se sigue que $\mathcal{V}$ tiene un refinamiento punto numerable $\mathcal{W}$. Ya que $X$ es débilmente estrella numerable, existe un subconjunto numerable $A \subseteq X$ tal que $\operatorname{cl}(S t(A, \mathcal{W}))=X$. Así para cada $x \in A$ existe una subfamilia numerable $\mathcal{W}_{x} \subseteq \mathcal{W}$ tal que $\bigcup\left\{W: W \in \mathcal{W}_{x}\right\}=S t(x, \mathcal{W})$. Por lo que tenemos:

$$
X=\operatorname{cl}(S t(A, \mathcal{W}))=\operatorname{cl}\left(\bigcup\left\{W: W \in \mathcal{W}_{x}, x \in A\right\}\right) .
$$

Como $\mathcal{W}$ es refinamiento de $\mathcal{V}$ tenemos que existe un subconjunto numerable $\left\{V_{n}: n \in\right.$ $\omega\}$ de $\mathcal{V}$ tal que $\bigcup\left\{V_{n}: n \in \omega\right\}$ es denso en $X$ y por lo tanto intersecta a cada $U \in \mathcal{U}$. Como cada $V_{n}$ intersecta a lo más a un número finito de elementos de $\mathcal{U}$, se sigue que $\mathcal{U}$ es numerable.

Teorema 5.3.7. Un espacio de Hausdorff metacompacto y débilmente estrella finita es $H$-cerrado (por lo tanto tenuemente compacto).

Demostración. Sea $X$ un espacio metacompacto Hausdorff y débilmente estrella finita y sea $\mathcal{U}$ una cubierta abierta de $X$. Como $X$ es metacompacto, se sigue que $\mathcal{U}$ tiene un refinamiento abierto punto finito $\mathcal{V}$. Como la propiedad débilmente estrella $P$ y casi estrella $P$ son equivalentes cuando $P$ es finito, tenemos que $X$ es casi estrella finita, se sigue que existe un subconjunto finito $F$ tal que $\bigcup\{\operatorname{cl}(S t(x, \mathcal{V}): x \in F\}=X$. Para cada $x \in F$, existe una subfamilia finita $\mathcal{V}_{x} \subseteq \mathcal{V}$ tal que:

$$
\bigcup\left\{\operatorname{cl}(V): V \in \mathcal{V}_{x}\right\}=\operatorname{cl}(S t(x, \mathcal{V}))
$$

Por lo tanto:

$$
X=\bigcup\{\operatorname{cl}(S t(x, \mathcal{V})): x \in F\}=\bigcup\left\{\operatorname{cl}(V): V \in \mathcal{V}_{x}, x \in F\right\}
$$

Como $\mathcal{V}$ es refinamiento de $\mathcal{U}$, se tiene que existe una subfamilia finita de $\mathcal{U}$ cuya unión es densa en $X$.

Teorema 5.3.8. Un espacio regular, metacompacto y casi estrella numerable es de Lindelöf.

Demostración. Sea $X$ un espacio regular, metacompacto y casi estrella numerable. Sea $\mathcal{U}$ una cubierta abierta de $X$, entonces existe un refinamiento abierto punto finito $\mathcal{V}$ tal que $\{\operatorname{cl}(U): U \in \mathcal{V}\}$ es un refinamiento de $\mathcal{U}$ por ser $X$ regular. Como $X$ es casi 
estrella numerable, existe un conjunto numerable $A \subseteq X$ tal que $\bigcup\{\operatorname{cl}(S t(x, \mathcal{V})): x \in$ $A\}=X$. Para cada $x \in A$, existe una subfamilia finita $\mathcal{V}_{x} \subseteq \mathcal{V}$ tal que:

$$
\bigcup\left\{\operatorname{cl}(U): U \in \mathcal{V}_{x}\right\}=\operatorname{cl}(S t(x, \mathcal{V}))
$$

Así tenemos que:

$$
X=\bigcup\{\operatorname{cl}(S t(x, \mathcal{V})): x \in A\}=\bigcup\left\{\operatorname{cl}(U): U \in \mathcal{V}_{x}, x \in A\right\}
$$

Se sigue que $\mathcal{U}$ tiene una subcubierta numerable.

Definición 5.3.9. Sea $(X, \tau)$ un espacio topológico y $\phi: X \rightarrow \tau$ con $x \in \phi(x)$ para cada $x \in X$, la función $\phi$ la llamaremos una asignación de vecindades para $X$. Decimos que $X$ es un espacio dualmente separable si para cada asignación de vecindades $\{\phi(x): x \in X\}$ existe un subespacio separable $Y \subseteq X$ tal que $\bigcup\{\phi(x): x \in Y\}=X$.

Definición 5.3.10. Un espacio $X$ es dualmente ccc si para cada asignación de vecindades $\{\phi(x): x \in X\}$ existe un subespacio ccc $Y \subseteq X$ tal que $\bigcup\{\phi(x): x \in Y\}=X$.

Teorema 5.3.11. Un espacio $X$ dualmente ccc es débilmente estrella numerable.

Demostración. Asumamos lo contrario, es decir, que existe una cubierta abierta $\mathcal{U}$ de $X$ tal que para cada subconjunto numerable $A \subseteq X$ tenemos que $\operatorname{cl}(S t(A, \mathcal{U})) \neq X$. Sea $x_{0} \in X$, para cada $\beta<\omega_{1}$ elegimos $x_{\beta} \in X \backslash \operatorname{cl}\left(\bigcup\left\{S t\left(x_{\alpha}, \mathcal{U}\right): \alpha<\beta\right\}\right)$ y sea :

$$
O_{\beta}=S t\left(x_{\beta}, \mathcal{U}\right) \backslash \operatorname{cl}\left(\bigcup\left\{S t\left(x_{\alpha}, \mathcal{U}\right): \alpha<\beta\right\}\right) .
$$

El subconjunto $O_{\beta}$ es abierto, $x_{\beta} \in O_{\beta}$ y $O_{\beta} \cap O_{\beta^{\prime}}=\emptyset$ cuando $\beta \neq \beta^{\prime}$. Sea $D=\left\{x_{\alpha}\right.$ : $\left.\alpha<\omega_{1}\right\}$. Como $\mathcal{U}$ es una cubierta de $X$ y cada $U \in \mathcal{U}$ contiene a lo más un elemento de $D$, se sigue que $D$ es cerrado y discreto en $X$.

Definimos $\phi: X \rightarrow \tau$ de la siguiente manera, para cada $x_{\alpha} \in D$ sea $\phi\left(x_{\alpha}\right)=O_{\alpha} \mathrm{y}$ para cada $x \in X \backslash D$ sea $\phi(x)=X \backslash D$. Como $X$ es dualmente ccc, para esta asignación de vecindades de $X$, existe un subespacio $Y \subseteq X$ que tiene la propiedad ccc tal que $\bigcup\{\phi(y): y \in Y\}=X$. Notemos que si $y \in D$, entonces $y \in \phi(x)$ si sólo si $y=x$; esto muestra que $D \subset Y$. Por lo tanto $\left\{O_{\alpha} \cap Y: \alpha<\omega_{1}\right\}$ es una familia ajena por pares no numerable de subconjuntos abiertos no vacíos de $Y$ lo que contradice que $Y$ es ccc.

Corolario 5.3.12. Un espacio dualmente separable es débilmente estrella numerable.

Demostración. Por el Lema 5.3.5 se sigue que cada espacio dualmente separable es dualmente ccc, por lo tanto es débilmente estrella numerable. 
Definición 5.3.13. Una familia $\left\{\mathcal{U}_{n}: n \in \omega\right\}$ de cubiertas abiertas de un espacio topológico $X$ se llama sucesión diagonal de orden $k$, donde $k \in \omega$, si para cada $x \in X$ se tiene que $\{x\}=\bigcap\left\{S t^{k}\left(x, \mathcal{U}_{n}\right): n \in \omega\right\}$. Decimos que un espacio $X$ tiene diagonal de orden $k$ si existe una sucesión diagonal de orden $k$ en $X$.

El siguiente teorema de Teoría de Conjuntos se debe a P. Erdös y a R. Rado, la demostración del siguiente lema la omitiremos ya que no es relevante para el presente trabajo, puede consultarla detalladamente en [11], A 4.4.

Teorema 5.3.14. Sea $X$ un conjunto con $|X|>\mathfrak{c}$ y supongamos que $[X]^{2}=\bigcup\left\{P_{n}\right.$ : $n \in \omega\}$. Entonces existen $n_{0} \in \omega$ y un subconjunto $S$ de $X$ con $|S|>\omega$ tal que $[S]^{2} \subseteq P_{n_{0}}$.

Teorema 5.3.15. Si $X$ es un espacio de Hausdorff débilmente estrella numerable con diagonal de orden 4 , entonces la cardinalidad de $X$ es a lo más $2^{\omega}$.

Demostración. Supongamos que $|X|>2^{\omega}$. Como $X$ tiene diagonal de orden 4 tiene, existe una sucesión $\left\{\mathcal{U}_{n}: n \in \omega\right\}$ de cubiertas abiertas de $X$ tales que para cada $x \in X$, tenemos $\{x\}=\bigcap\left\{S t^{4}\left(x, \mathcal{U}_{n}\right): n \in \omega\right\}$. Para cada $n \in \omega$ definimos $P_{n}=\left\{\{x, y\} \in[X]^{2}: x \notin S t^{4}\left(y, \mathcal{U}_{n}\right)\right\}$.

Veamos que $[X]^{2}=\bigcup\left\{P_{n}: n \in \omega\right\}$. Sea $\{x, y\} \in[X]^{2}$ y supongamos que $x \in S t^{4}\left(y, \mathcal{U}_{n}\right)$ para cada $n \in \omega$. Entonces $x \in \bigcap\left\{S t^{4}\left(y, \mathcal{U}_{n}\right): n \in \omega\right\}=\{y\}$, así $x=y$; lo cual es una contradicción; así $[X]^{2}=\bigcup\left\{P_{n}: n \in \omega\right\}$. Por el Teorema 5.3.14 existe un subconjunto no numerable $T$ en $X$ tal que $[T]^{2} \subseteq P_{n_{0}}$ para algún $n_{0} \in \omega$.

Notemos los siguientes hechos:

(1) $\operatorname{cl}\left(S t\left(T, \mathcal{U}_{n_{0}}\right)\right) \subseteq S t^{2}\left(T, \mathcal{U}_{n_{0}}\right)$ por el Lema 2.1.2.

(2) $\bigcup\left\{\operatorname{cl}\left(S t\left(x, \mathcal{U}_{n_{0}}\right)\right): x \in T\right\}=\operatorname{cl}\left(S t\left(T, \mathcal{U}_{n_{0}}\right)\right)$. Para ver esto es suficiente mostrar que $\left\{S t\left(x, \mathcal{U}_{n_{0}}\right): x \in T\right\}$ no tiene punto de acumulación en $X$. Si $y$ fuera un punto de acumulación de la familia $\left\{S t\left(x, \mathcal{U}_{n_{0}}\right): x \in T\right\}$, entonces por (1) tendríamos que $y \in S t^{2}\left(T, \mathcal{U}_{n_{0}}\right)$. Por lo que existe $x_{0} \in T$ tal que $y \in S t^{2}\left(x_{0}, \mathcal{U}_{n_{0}}\right)$. De esto podemos deducir que si $x \in T \backslash\left\{x_{0}\right\}$ tenemos que $S t^{2}\left(x_{0}, \mathcal{U}_{n_{0}}\right) \cap S t\left(x, \mathcal{U}_{n_{0}}\right)=\emptyset$ pues de otro modo $x \in S t^{3}\left(x_{0}, \mathcal{U}_{n_{0}}\right)$ lo que contradice la elección del conjunto $T$. Esto muestra que $y$ no es punto de acumulación de $\left\{S t\left(x, \mathcal{U}_{n_{0}}\right): x \in T\right\}$.

(3) $\left\{S t^{2}\left(x, \mathcal{U}_{n_{0}}\right): x \in T\right\}$ es ajeno por pares. Supongamos que existen dos puntos distintos $x, y \in T$ tales que $\left.S t^{2}\left(x, \mathcal{U}_{n_{0}}\right) \cap S t^{2}\left(y, \mathcal{U}_{n_{0}}\right)\right) \neq \emptyset$, se sigue que $x \in S t^{4}\left(y, \mathcal{U}_{n_{0}}\right)$, por lo que $\{x, y\} \notin P_{n_{0}}$. Como $x, y \in T$ tenemos que $\{x, y\} \in[T]^{2} \subseteq P_{n_{0}}$ lo cual es una contradicción.

Ahora por (1) podemos definir la siguiente cubierta abierta de $X$ dada por:

$$
\mathcal{U}=\left\{S t^{2}\left(x, \mathcal{U}_{n_{0}}\right): x \in T\right\} \cup\left\{X \backslash \operatorname{cl}\left(S t\left(T, \mathcal{U}_{n_{0}}\right)\right)\right\} .
$$


Si $A \subseteq X$ es un subconjunto numerable, entonces como cada punto de $A$ está en a lo más a dos elementos de $\mathcal{U}$ por (3), existe $\xi \in T$ tal que $A \cap S t^{2}\left(\xi, \mathcal{U}_{n_{0}}\right)=\emptyset$. Se sigue que si $\eta \in \operatorname{cl}\left(S t\left(\xi, \mathcal{U}_{n_{0}}\right)\right)$, entonces $\eta \notin X \backslash \operatorname{cl}\left(S t\left(T, \mathcal{U}_{n_{0}}\right)\right)$ por $(2)$, y así cl $(\bigcup\{S t(T, \mathcal{U})$ : $x \in A\}) \neq X$. Esto contradice que $X$ es un espacio débilmente estrella numerable.

Corolario 5.3.16. Si $X$ es un espacio de Hausdorff casi estrella numerable con diagonal de orden 4 , entonces la cardinalidad de $X$ es a lo más $2^{\omega}$.

En [23] se demuestra el siguiente resultado:

Proposición 5.3.17. Si $X$ es un espacio débilmente regular y débilmente estrella numerable con diagonal de orden 3 , entonces la cardinalidad de $X$ es a lo más $2^{\omega}$.

Una pregunta interesante que proponen Wei-Feng Xuan y Yan-Kui Song en [23] es:

Pregunta 5.3.18. ¿Es cierto que cada espacio de Hausdorff débilmente estrella numerable (o casi estrella numerable) con diagonal de orden 3 tiene cardinalidad a lo más $2^{\omega} ?$ 


\section{Capítulo 6}

\section{Conclusiones}

Finalmente podemos exponer los resultados más importantes estudiados a lo largo de este trabajo y los temas que se pueden abordar a partir de este conocimiento.

En Capítulo 2 se demostró:

1) Cualquier espacio topológico numerablemente compacto es estrella finito, pero la implicación recíproca no se cumple ya que Ejemplo 2.1.17 es un espacio $T_{1}$ estrella finito que no es numerablemente compacto. Para obetener la equivalencia de estas dos propiedades topológicas basta que el espacio sea de Hausdorff.

2) Cualquier espacio topológico estrella compacto es 1-estrella finito, pero la implicación recíproca no se cumple y el Ejemplo 2.1.23 es prueba de ello.

3) En espacios de Tychonoff cada espacio estrella finito de orden $\omega$ es pseudocompacto.

4) Cada espacio tenuemente compacto es 2-estrella finito.

Otros resultados importantes:

1) Cada espacio separable es estrella numerable;

2) Cada espacio de Lindelöf tiene extensión numerable;

3) Un espacio es estrella numerable si y sólo si es estrella separable;

4) El producto de un espacio estrella Lindelöf y un espacio compacto es estrella Lindelöf;

5) El producto de un espacio tenuemente Lindelöf y un espacio separable es tenue- 
mente Lindelöf.

En el Capítulo 3 se demostró que en espacios $T_{1}$ :

1) Cada espacio $\omega_{1}$-Lindelöf tiene extensión numerable, pero la implicación recíproca no se cumple ya que $\omega_{1}$ con la topología del orden tiene extensión numerable pero no es $\omega_{1}$-Lindelöf.

2) Cada espacio con extensión numerable es estrella numerable, pero la implicación recíproca no se cumple ya que el espacio de Mröwka $\Psi(\mathcal{A})=\omega \cup \mathcal{A}$ es separable y tiene extensión no numerable.

3) Cada espacio estrella numerable es estrella $\sigma$-compacto, pero la implicación recíproca no se cumple y el Ejemplo 3.1.23 es prueba de esto.

4) Cada espacio estrella $\sigma$-compacto es estrella Lindelöf, pero la implicación recíproca no se cumple y el Ejemplo 3.1.5 es prueba de esto.

5) Cada espacio estrella Lindelöf es $\mathbf{S C E}$, pero la implicación recíproca no se cumple y el Ejemplo 3.2.7 es prueba de esto.

6) Cada espacio SCE es tenuemente Lindelöf.

El siguiente diagrama resume lo expuesto en estos seis puntos

$$
\begin{gathered}
\omega_{1} \text {-Lindelöf } \Rightarrow \text { Extensión numerable } \Rightarrow \text { Estrella numerable } \Rightarrow \text { Estrella } \\
\quad \sigma \text {-compacto } \\
\Rightarrow \text { Estrella Lindelöf } \Rightarrow \text { SCE } \Rightarrow \text { Tenuemente Lindelöf. }
\end{gathered}
$$

Para obtener algunas implicaciones recíprocas necesitamos que el espacio sea normal y $P$-espacio; así tenemos las siguientes equivalencias:

Extensión numerable $\Leftrightarrow$ Estrella numerable $\Leftrightarrow$ Estrella $\sigma$-compacto $\Leftrightarrow$ Estrella Lindelöf $\Leftrightarrow \mathrm{SCE} \Leftrightarrow$ Tenuemente Lindelöf.

En la Sección 3.1 se demostró la existencia de un $P$-espacio de Tychonoff tenuemente Lindelöf que no es estrella Lindelöf bajo la suposición de que existe una familia $M C A G \mathcal{A}$ en $\omega_{1}$ tal que $\left|\mathcal{A}^{\omega}\right|=|\mathcal{A}|$, esta suposición es independiente de ZFC y es un ejercicio en [14]. Dos preguntas interesantes a partir de esto son:

1) ¿Es consistente con ZFC que el Ejemplo 3.1.30 es estrella Lindelöf? 
2) ¿Un P-espacio tenuemente Lindelöf es estrella Lindelöf?

En Capítulo 4 se demostró que si $X$ es un $P$-espacio normal, entonces las siguientes propiedades son equivalentes:

\section{Estrella numerable $\Leftrightarrow$ Estrella Lindelöf $\Leftrightarrow$ Tenuemente Lindelöf $\Leftrightarrow X$ tiene la dccc $\Leftrightarrow$ Extensión numerable.}

También se demostraron las siguientes equivalencia en espacios de Moore:

$$
\text { Estrella numerable } \Leftrightarrow \text { Estrella Lindelöf } \Leftrightarrow \text { Separable }
$$

En [18] se demuestra que en espacios de Moore la propiedad SCE también es equivante a la separabilidad y también se proporciona un ejemplo de un espacio de Moore tenuemente Lindelöf que no es $S C E$.

En Capítulo 5 se demostraron los siguientes resultados:

1) Cualquier espacio topológico casi estrella $P$ es débilmente estrella $P$.

2) Cualquier espacio topológico $T_{1}$ con la $\mathbf{D C}\left(\omega_{1}\right)$ es débilmente estrella numerable.

3) Cada espacio con celularidad numerable es débilmente estrella numerable.

4) Cada espacio estrella numerable es casi estrella numerable, pero la implicación recíproca no se cumple ya que el Ejemplo 5.1.18 es prueba de ello.

En Capítulo 5 también se demostró que si $X$ es un $P$-espacio normal, entonces las siguientes propiedades son equivalentes:

\section{Débilmente estrella numerable $\Leftrightarrow$ Casi estrella numerable $\Leftrightarrow$ $X$ tiene un subespacio denso con extensión numerable $\Leftrightarrow$ estrella numerable.}

Otros resultados importantes del capítulo 5 son:

1) Un espacio de Hausdorff metaLindelöf y débilmente estrella numerable es tenuemente Lindelöf. 
2) Un espacio de Hausdorff metacompacto y débilmente estrella finita es H-cerrado.

3) Un espacio regular, metacompacto y casi estrella numerable es de Lindelöf.

4) Un espacio dualmente ccc es débilmente estrella numerable.

5) Un espacio de Hausdorff débilmente estrella numerable con diagonal de orden 4 tiene cardinalidad a lo más $2^{\omega}$. 


\section{Bibliografía}

[1] L. P. Aiken, Star-covering properties: Gereneralized $\Psi$-spaces, countability conditions, reflection, Topology and its Applications, 158 (2011), 1732-1737.

[2] O. T. Alas, L. R. Junqueira, V. V. Tkachuk, R. G. Wilson, On the extent of star countable spaces, Central European Journal Of Mathematics, 9(3) (2011), 603-615.

[3] O. T. Alas, L. R. Junqueira, R. G. Wilson, Countability and star covering properties, Topology and its Applications, 158 (2011), 620-626.

[4] O. T. Alas, R. G. Wilson, Properties related to star countability and star finiteness, Topology and its Applications, 224 (2017), 432-439.

[5] E. K. van Douwen, G. M. Reed, A. W. Roscoe, I. J. Tree, Star covering properties, Topology and its Applications, 39 (1991), 71-103.

[6] R. Engelking, General Topology, Heldermann Verlag, Berlín, Alemania, 1989.

[7] L. Gillman, M. Jerison, Rings of continuous functions, D.Van Nostrand Company, Nueva York, EUA, 1960.

[8] M. Hrušák, A. Tamariz, M. Tkachenko, Pseudocompact Topological Spaces, Springer, 2018.

[9] S. Ikenaga, Topologial concepts between Lindelöf and pseudo-Lindelöf, Res.Rep.Nara Nat. College Tech, 26 (1990), 103-108.

[10] S. Ikenaga, A class which contains Lindelöf spaces, separable spaces and countably compact spaces, Memoirs of Numazu College of Technology, 18 (1983), 105-108.

[11] I. Juhász, Cardinal Functions in Topology, Mathematisch Centrum Amsterdam, 1971.

[12] N. Kemoto, H. Ohta, K. Tamano, Products of spaces of ordinal numbers, Topology and its Applications, 45 (1992), 245-260. 
[13] N. Kemoto, K. D. Smith, P. J. Szeptycki, Countable paracompactness versus normality in $\omega_{1}^{2}$, Topology and its Applications, 104 (2000), 141-154.

[14] K. Kunen, Set Theory, North-Holland, Amsterdam, 1980.

[15] K. Kuratowski, Topology, Academic Press, New York, 1966.

[16] M. V. Matveev, A survey on star covering properties, Topology Atlas preprint, April 15, 1998.

[17] J. van Mill, V. V. Tkachuk, R. G. Wilson, Clasess defined by stars and neighbourhood assignments, Topology and its Applications, 154 (2007), 2127-2134.

[18] A. D. Rojas, A. Tamariz, Spaces with star countable extent, Commentationes Mathematicae Universitatis Carolinae, 57(3) (2016), 381-395.

[19] L. A. Steen, J. A. Seebach, Jr, Counterexamples in topology, New York, 1970.

[20] Y. K. Song, Some remarks on almost star countable spaces, Studia Scientiarum Mathematicarum Hungarica, 52(1) (2015), 12-20.

[21] Y. K. Song, Star covering properties in pseudocompact spaces, Topology and its Applications, 159 (2012), 1462-1466.

[22] Y. K. Song, W. F. Xuan, Remarks on weakly star countable spaces, Matematiĉki Vesnik, 70(3) (2018), 250-256.

[23] Y. K. Song, W. F. Xuan, On properties related to star countability. Topology and its Applications, 247 (2018), 20-28.

[24] R. C. Walker, The Stone-ĈCeh Compactification. Springer-Verlag, Nueva York, EUA, 1974.

[25] R. G. Wilson, R. Benitez, Topología general, Trillas, Ciudad de México, México, 2017.

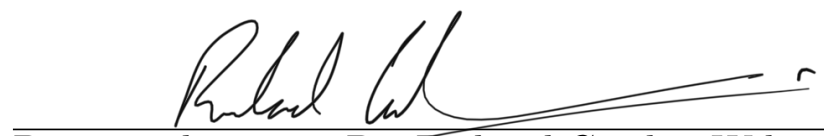

Director de tesis. Dr. Richard Gordon Wilson Roberts 
Propiedades topologicas del tipo estrella

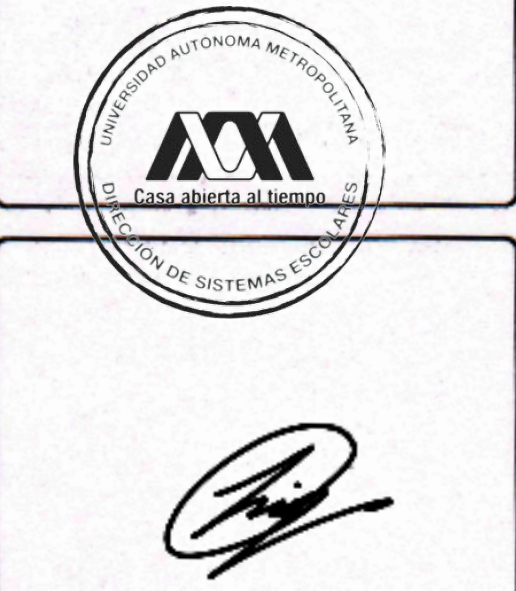

LUIS ENRIQUE GUTIERREZ DOMINGUEZ

ALUMENO

REVISO

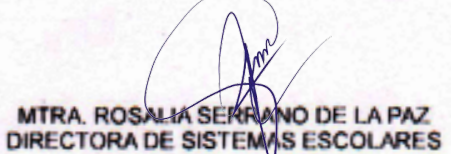

Con base en la Legislación de la Universidad Autonoma Metropolitana, en la Cludad de Mexico se presentaron a las 13:00 horas del dia 27 del mes de octubre del año 2020 POR VIA REMOTA ELECTRÓNICA, los suscritos miembros del jurado designado por la Comisión del Posgrado:

- dr. anget tamariz mascarua

DR. RICHARD GORDON WILSON ROBERTS

DR. RODRIGO JESUS HERNANDEZ GUTIERREZ

Bajo la Presidencia del primero y con carácter de Secretario el ultimo. se reunieron para proceder al Examen de Grado cuya denominación aparece al margen, para la obtención del grado de:

MAESTRO EN CIENCIAS (MATEMÁTICAS)

DE: LUIS ENRIQUE GUTIERREZ DOMINGUE2

$y$ de acuerdo con el articulo 78 fracción II de 1 Reglamento de Estudios Superiores de la Universidad Autonoma Metropolitana, los miembros del jurado resolvieron:

\section{APROBAR}

Acto continuo, el presidente del jurado comunicó al intereaado el resultado de la evaluacion $y$, en caso aprobatorio, le fue tomada la protesta.

El presente documento cuenta con la firma -autógrafa, escaneada o digital, según corresponda- del funcionario universitario competente, que certifica que las firmas que aparecen en esta acta - Temporal, digital o dictamen- son auténticas y las mismas que usan los c.c. profesores mencionados en ella 